\title{
Search for sterile neutrinos with the Neutrino-4 experiment and measurement results
}

A. P. Serebrov $\odot,{ }^{1,}{ }^{*}$ R. M. Samoilov, ${ }^{1}$ V. G. Ivochkin, ${ }^{1}$ A. K. Fomin, ${ }^{1}$ V. G. Zinoviev, ${ }^{1}$ P. V. Neustroev, ${ }^{1}$ V. L. Golovtsov, ${ }^{1}$ S. S. Volkov, ${ }^{1}$ A. V. Chernyj, ${ }^{1}$ O. M. Zherebtsov, ${ }^{1}$ M. E. Chaikovskii, ${ }^{1}$ A. L. Petelin, ${ }^{2}$ A. L. Izhutov, ${ }^{2}$ A. A. Tuzov, ${ }^{2}$ S. A. Sazontov, ${ }^{2}$ M. O. Gromov, ${ }^{2}$ V. V. Afanasiev, ${ }^{2}$ M. E. Zaytsev, ${ }^{1,3}$ A. A. Gerasimov, ${ }^{1}$ and V. V. Fedorov ${ }^{1}$

${ }^{1}$ National Research Center Kurchatov Institute-Petersburg Nuclear Physics Institute, 188300 Gatchina, Russia

${ }^{2}$ JSC “State Science Center Research Institute of Atomic Reactors", 433510 Dimitrovgrad, Russia

${ }^{3}$ Dimitrovgrad Engineering and Technological Institute MEPhI, 433511 Dimitrovgrad, Russia

(Received 22 May 2020; revised 5 April 2021; accepted 15 June 2021; published 17 August 2021)

The experiment Neutrino-4 started in 2014 with a detector model and continued with a full-scale detector in 2016-2021. In this article, we describe all the steps of the preparatory work on this experiment. We present all results of the Neutrino-4 experiment with increased statistical accuracy provided to date. The experimental setup is constructed to measure the flux and spectrum of the reactor antineutrinos as a function of distance to the center of the active zone of the SM-3 reactor (Dimitrovgrad, Russia) in the range of 6-12 meters. Using all the collected data, we performed a model-independent analysis to determine the oscillation parameters $\Delta \mathrm{m}_{14}^{2}$ and $\sin ^{2} 2 \theta_{14}$. The method of coherent summation of measurement results allows us to directly demonstrate the oscillation effect. We present the analysis of possible systematic errors and the MC model of the experiment, which considers the possibility of the effect manifestation at the present precision level. As a result of the analysis, we can conclude that at currently available statistical accuracy, we observe the oscillations at the $2.9 \sigma$ level with the parameters $\Delta m_{14}^{2}=\left(7.3 \pm 0.13_{\text {st }} \pm 1.16_{\text {syst }}\right) \mathrm{eV}^{2}=(7.3 \pm 1.17) \mathrm{eV}^{2}$ and $\sin ^{2} 2 \theta=0.36 \pm 0.12_{\text {stat }}(2.9 \sigma)$. Monte Carlo based statistical analysis gave an estimation of the confidence level at $2.7 \sigma$. We plan to improve the currently working experimental setup and create a completely new setup in order to increase the accuracy of the experiment by 3 times. We also provide a brief analysis of the general experimental situation in the search for sterile neutrinos.

DOI: $10.1103 /$ PhysRevD.104.032003

\section{INTRODUCTION}

Experiments on the search for possible neutrino oscillations in a sterile state have been carried out for many years. There are experiments at accelerators, reactors, and artificial neutrino sources [1-28]. A sterile neutrino can be considered as a candidate for the dark matter particles.

By combining the results of various reactor experiments, one can estimate the ratio of the observed antineutrino flux to the predicted flux to be $0.927 \pm 0.023$ [29-32]. The deviation from the no oscillation hypothesis is about 3 standard deviations. This level is not yet sufficient to have confidence in the existence of the reactor antineutrino anomaly. Importantly, the method to test the hypothesis of oscillation into a sterile state, in which one compares the

*serebrov_ap@pnpi.nrcki.ru

Published by the American Physical Society under the terms of the Creative Commons Attribution 4.0 International license. Further distribution of this work must maintain attribution to the author(s) and the published article's title, journal citation, and DOI. Funded by SCOAP ${ }^{3}$. measured antineutrino flux with the expected flux from the reactor is not satisfactory, because of the problems with the accurate estimation of both a reactor antineutrino flux and efficiency of an antineutrino detector. The possible process of oscillations to a sterile state at small distances from an active zone of the reactor is shown in Fig. 1, which was published in [32].

If the oscillation process does exist, then the deviation of antineutrino flux from the flux calculated in the assumption of no oscillation can be described by the equation,

$P\left(\bar{\nu}_{e} \rightarrow \bar{\nu}_{e}\right)=1-\sin ^{2} 2 \theta_{14} \sin ^{2}\left(1.27 \frac{\Delta \mathrm{m}_{14}^{2}\left[\mathrm{eV}^{2}\right] \mathrm{L}[\mathrm{m}]}{\mathrm{E}_{\bar{\nu}}[\mathrm{MeV}]}\right)$

where $E_{\bar{\nu}}$ is antineutrino energy in $\mathrm{MeV}, \mathrm{L}$ is the distance in meters, $\Delta \mathrm{m}_{14}^{2}$ is the difference between squared masses of electron and sterile neutrinos, and $\theta_{14}$ is the mixing angle of an electron and sterile neutrinos. The experimental test of the oscillation hypothesis requires measurements of the 
- Observed/predicted averaged event ratio: $R=0.927 \pm 0.023(3.0 \sigma)$

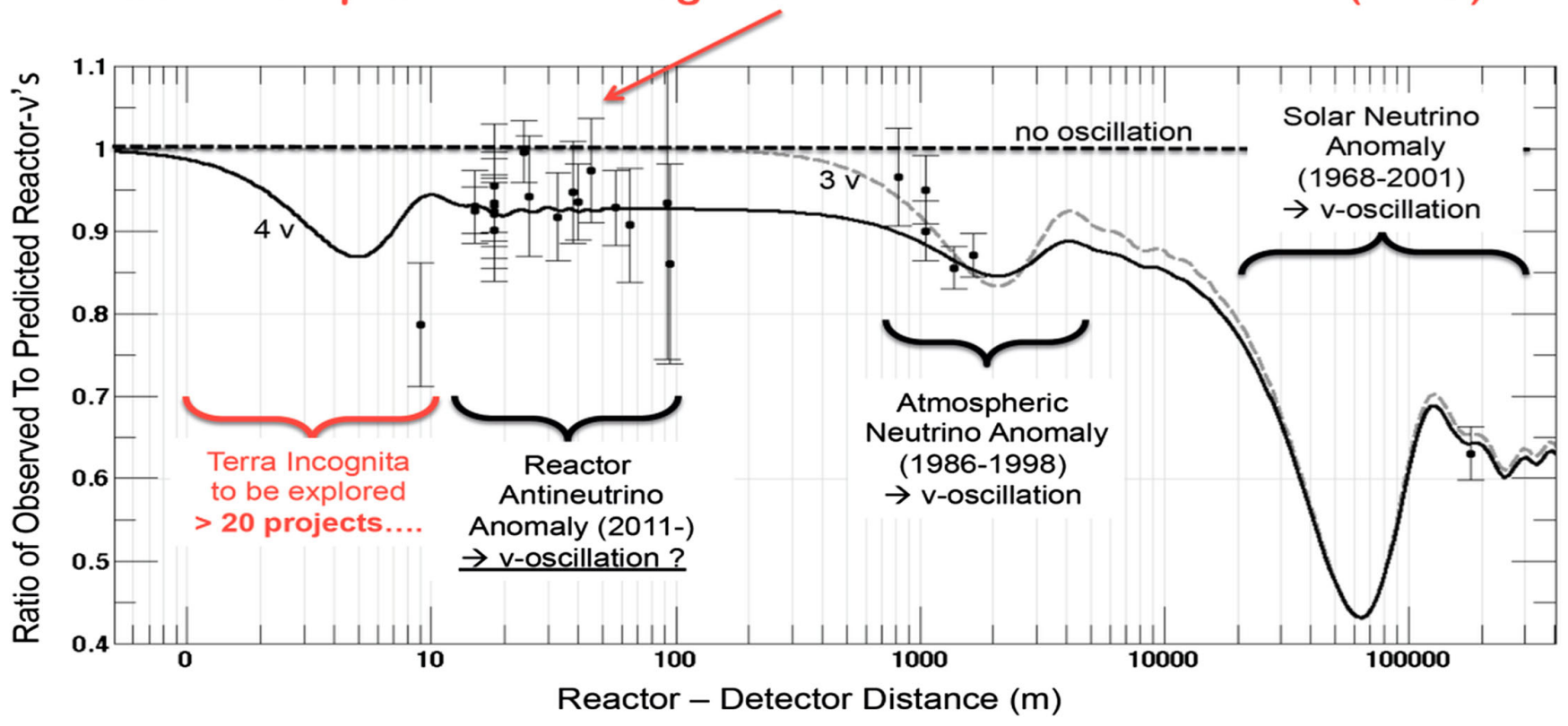

FIG. 1. The possible process of oscillations to a sterile state at small distances from an active zone of the reactor.

antineutrino flux and spectrum as near as possible to a practically pointlike antineutrino source.

Based on Eq. (1), the oscillation hypothesis can be verified by the direct measurement of distance dependences of antineutrino flux and spectrum at as short as possible distances to a practically pointlike antineutrino source. The oscillations manifest themselves in two effects: the way neutrino flux varies with distance deviate from the quadratic decrease form $1 / \mathrm{L}^{2}$, and the neutrino spectrum varies with distance. Therefore, a neutrino detector has to be movable and a spectrum sensitive. Our experiment focuses on the task of confirming the possible existence of a sterile neutrino at a certain confidence level or disproving it. We have studied the potential of research reactors in Russia to conduct new experiments. The research reactors should be employed for performing such experiments, since they possess a compact reactor core, so that a neutrino detector can be placed at a sufficiently small distance from it. Unfortunately, research reactor beam halls have quite a large background of neutrons and gamma quanta from the operating reactor, which makes it difficult to perform low background experiments. Due to some peculiar characteristics of its construction, reactor SM-3 provides the most favorable conditions for conducting an experiment on the search for neutrino oscillations at small distances. At the same time, the SM-3 reactor, like other research reactors, is located on the Earth's surface; hence, an experimental setup of a neutrino experiment is exposed to high cosmic background, and it appears to be the major difficulty for the experiment.

Here is the structure of the article and brief description of the sections.
The first part of the article describes preparations for the experiment, which include the study of the background at the reactor SM-3. The antineutrino signal should be selected from the background produced by cosmic rays, which is inevitably present. Antineutrino penetrates the biological shielding of the reactor without weakening. The method of the antineutrino signal selection is to compare the results obtained with the operating and stopped reactor. This is the so-called ONOFF count rate. That method can be applied if the background conditions in the vicinity of the antineutrino detector do not change when the reactor is switched between operating and stopped states. The most dangerous source of the background is the fast neutrons, which can simulate the antineutrino registration signal. This issue is addressed in Secs. II-V, where we present measurements carried out with the detector of fast neutrons, background suppression, building of the passive shielding, and investigation of its characteristics.

Studies of the cosmic ray background were carried out with a test model of a neutrino detector filled with 400 liters of a gadolinium-doped liquid scintillator $(0.1 \% \mathrm{Gd})$ and covered with the active shielding against cosmic muons. They are presented in Secs. VI and VII. In these studies, the measurements of fluctuations of the cosmic rays background with time inside the neutrino channel (passive shielding) were obtained where the antineutrino detector moves. The model of the detector was applied to investigate the method of antineutrino registration as a result of the inverse beta decay (IBD) process as well as the effectiveness of active shielding to suppress the background of cosmic rays.

The next stage of research (Sec. VIII) was carried out on a sectioned model of the detector, also as a next preparatory stage for the creation of the full-scale detector. Here, an 
attempt has been made to separate the neutrino signal from the fast neutron signal, relying on the fact that in the inverse beta decay reaction, there are two gamma quanta $(511 \mathrm{keV})$ that can be recorded in adjacent sections. Also, we present the results of studies with a single section.

Sections IX-XVI are devoted to the full-scale detector: energy calibration of the detector, the computer model of the detector, obtaining the spectrum of antineutrino signals, and comparing it to the calculated spectrum.

In Secs. XVII-XIX, we discuss the spectrum independent method for analysis of the neutrino signal and the Monte Carlo simulations of this method. This is an extremely important point that allows us to move to real measurements and to process the results in order to reveal possible neutrino oscillations at short distances.

Sections $\mathrm{XX}-\mathrm{XXV}$ are devoted to the results of the measurements, their analysis, searching for the oscillation signal, and the statistical and systematic uncertainties of the oscillation effect.

In Sec. XXVI, we describe MC modeling of the experiment, which takes into consideration the obtained statistical accuracy.

The final part of the article (Sec. XXVII) is devoted to the comparison of the results of this study with the results of other experiments. This analysis is of great importance because it ensures that the obtained result does not contradict the results of other experiments on the search for sterile neutrinos.

\section{REACTOR SM-3}

Initially, the SM-3 reactor having maximum power $100 \mathrm{MW}$ was designed for carrying out both beam and loop experiments. Five beam halls were built and separated from each other with $1 \mathrm{~m}$ wide concrete walls (Fig. 2).

This enabled the carrying out of experiments on neutron beams, without changing background conditions at neighboring installations. Later on, the main experimental program was focused on the tasks concerned with irradiation in the reactor core center. For 25 years of exploitation, a significantly high fluence was accumulated in materials of the reactor pressure vessel, which necessitated its replacement. Setting a new reactor pressure vessel on old reactor core barrel without joints with horizontal reactor beamlines was the simplest way to solve this problem. This decision led to raising of the reactor core center by $67 \mathrm{~cm}$ relative to the previous position. As a result, horizontal beamlines ceased to be used, as priority was given to conducting loop experiments. Neutron flux in the location of the former beamlines was lowered by 4 orders of magnitude. Therefore, neutron background (thermal neutrons) decreased to a level of about $4 \times 10^{-3} \mathrm{n} /\left(\mathrm{cm}^{2} \mathrm{~s}\right)$ in the former beam halls. It is several orders of magnitude lower than a typical neutron background in the beam hall of a research reactor.

Besides the favorable level of the reactor background, another advantage of the SM-3 reactor is its compact reactor core $\left(35 \times 42 \times 42 \mathrm{~cm}^{3}\right)$, with a high reactor power equal to $90 \mathrm{MW}$.

In one of the former beam halls, we created a laboratory to carry out an experiment on the search for oscillation of a reactor antineutrino into a sterile state. This hall fulfills the conditions important to our experiment: small distance $(5 \mathrm{~m})$ from the reactor core to the wall of the hall and the size of the hall enables us to carry out measurements of antineutrino flux in a wide range 6-12 $\mathrm{m}$.

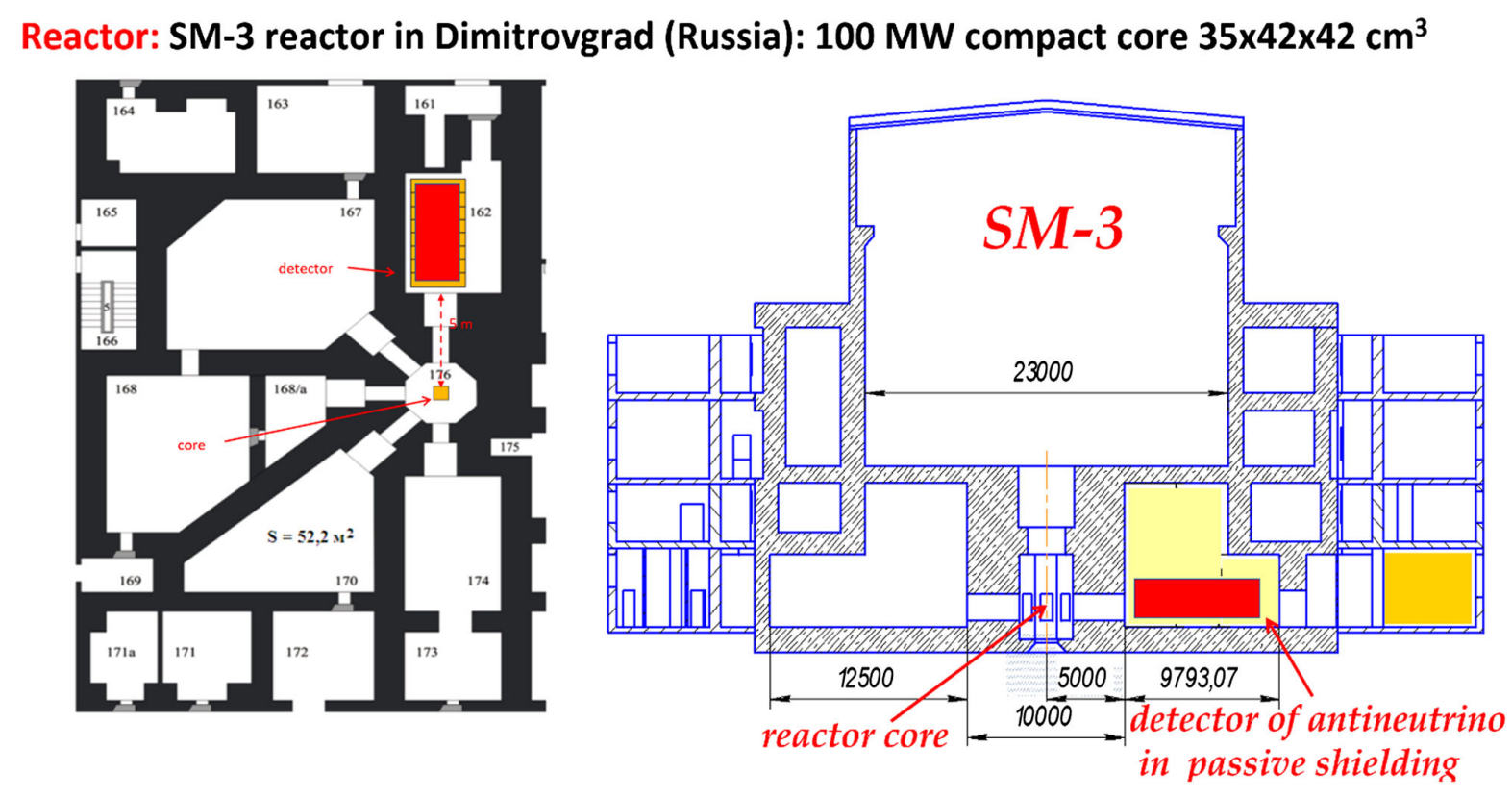

FIG. 2. Detector location at the SM-3 reactor. 
In making the preparations of the laboratory, the slide valve of the former neutron beamline has been upgraded to close all possible gaps to stop neutrons and gammas. As a result, the background of fast neutrons has decreased to the level of $10^{-3} \mathrm{n} /\left(\mathrm{cm}^{2} \mathrm{~s}\right)$, i.e., practically, to the level of neutron background on the Earth's surface, caused by cosmic rays. Achieved conditions can be considered as the most favorable of all possible for an experiment on the search for neutrino oscillation at small distances.

Up to $10^{3}$ neutrino events are expected to occur per day, at the reactor with nominal power of $90 \mathrm{MW}$ at an $8 \mathrm{~m}$ distance from the reactor core center, with a detector volume of $1 \mathrm{~m}^{3}$. However, registration efficiency in our method is only about $30 \%$, so with $1 \mathrm{~m}^{3}$ of liquid scintillator, we can record about 300 antineutrino events per day. This event rate is considered to be not very high, but it is sufficient to carry out an experiment with cosmic background conditions. The scheme of the antineutrino detector placement at the SM-3 reactor is shown in Fig. 2.

\section{PASSIVE SHIELDING OF ANTINEUTRINO DETECTOR AT THE SM-3 REACTOR}

To carry out a neutrino experiment at a research reactor, a detector has to be placed into passive shielding to protect it from the background. In order to determine optimal parameters for the shielding, the background conditions of the experiment were minutely investigated. Descriptions and results are presented in the following sections.

In order to fulfill requirements of the experiment and bring the background conditions to an acceptable level, the passive shielding ("cabin") was constructed and its image from the outside and inside is shown in Fig. 3. The shielding is made of elements based on steel plates of size $1 \times 2 \mathrm{~m}, 10 \mathrm{~mm}$ thick, to which are attached six lead sheets of $10 \mathrm{~mm}$ thickness. The cabin volume is $2 \times 2 \times 8 \mathrm{~m}$.
From the inside, the cabin is covered with plates of borated polyethylene of $16 \mathrm{~cm}$ thickness. The total weight of passive shielding is 60 tons, the volume of borated polyethylene is $10 \mathrm{~m}^{3}$. Inside the passive shielding, there is a platform with an antineutrino detector, which can be moved along the rails within the range 6-12 $\mathrm{m}$ from the center of the reactor core. The cabin (the neutrino beamline) can be entered by means of a ladder, through the roof with the removed upper unit, as shown in Fig. 3. The main hall of the reactor and our experimental hall are connected by a trapdoor in the celling of the hall. Loading of the detector into a neutrino beamline is carried out from the main hall through this trapdoor. In this case, an overhead crane of the main hall is used.

\section{INVESTIGATION OF BACKGROUND CONDITIONS INSIDE AND OUTSIDE OF PASSIVE SHIELDING WITH A GAMMA DETECTOR}

The detailed knowledge of background conditions around the detector are absolutely necessary to carry out the neutrino experiment. We performed the detailed investigation of various types of backgrounds. Flux and spectrum of gamma particles was measured with a detector based on a $\mathrm{NaI}(\mathrm{Tl})$ crystal of size $60 \times 400 \mathrm{~mm}$.

The first measurements of the gamma background in the neutrino laboratory hall were carried out before the installation of passive shielding. While the reactor was in operation mode, we registered gammas from neutron capture in an iron-concrete shielding of the reactor. During the reactor operation, the background of gamma radiation, in the energy range from $3 \mathrm{MeV}$ to $8 \mathrm{MeV}$ significantly (22 times larger than natural radiation background) increased, because of thermal neutrons interaction with the iron shot contained in concrete shielding of the reactor. This energy range is of great importance, since it
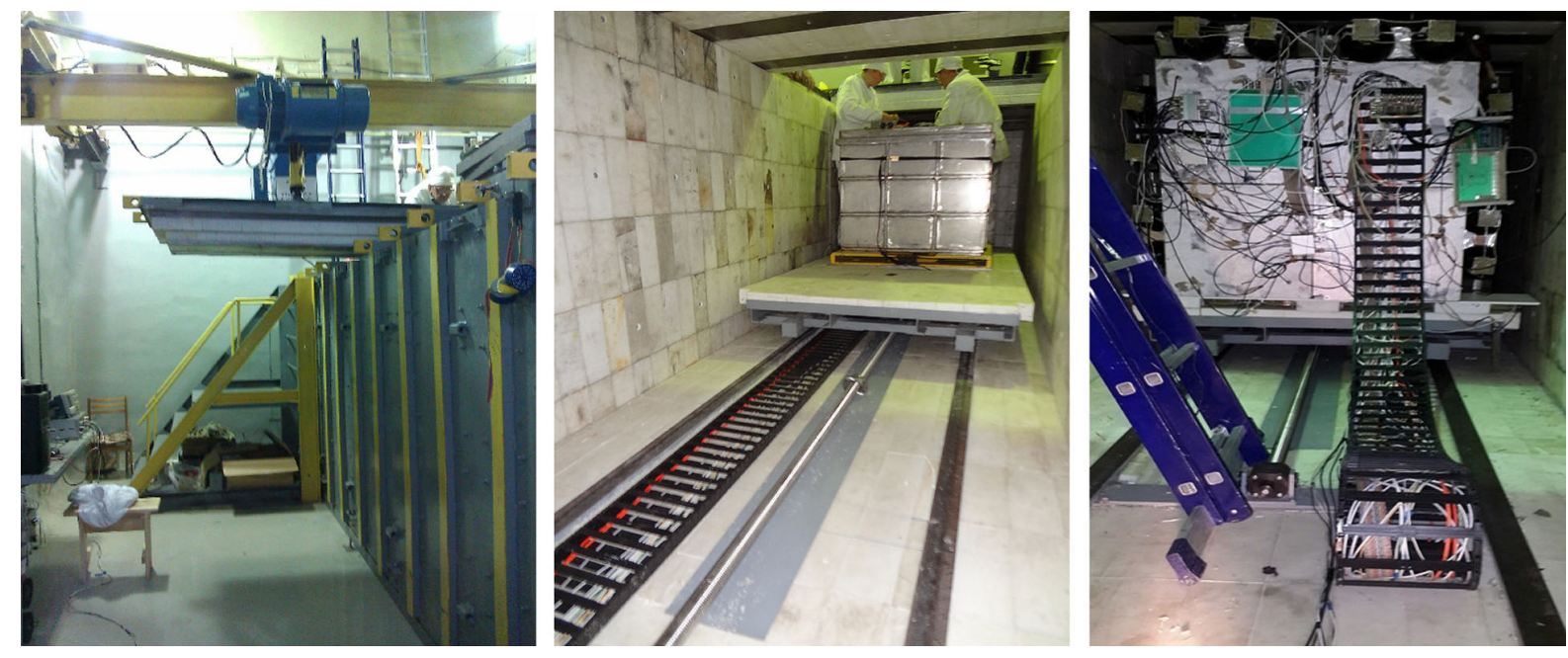

FIG. 3. General view of passive shielding: from the outside and inside. The range of detector movements is 6-12 $\mathrm{m}$ from the center of the reactor core on the left. Model detector is in the center; full-scale detector is on the right. 
corresponds to energy of gamma-quanta emitted in the process of neutron capture by Gd, which we use to register antineutrinos in our detector.

Gamma radiation of the isotopes ${ }^{137} \mathrm{Cs}$ and ${ }^{60} \mathrm{Co}$ is independent of the reactor operation mode and is caused by radioactive contamination from the building floor and walls. Concrete reinforced with iron grit was used for flooring, and the slide valve was reconstructed. These modifications reduced the gamma radiation background 5-6 times in the energy range we are concerned with. Despite that, remaining the gamma background was still too high, and it confirmed the necessity of the creation of passive shielding from gamma rays for the detector. Installation of passive shielding significantly suppressed the gamma background from the reactor to the level of radioactive contamination in the passive shielding.

Within the energy range of $1440 \div 7200 \mathrm{keV}$ (from the ${ }^{40} \mathrm{~K}$ line and higher), the $5 \mathrm{~cm}$ lead shielding makes the level of background gamma radiation 4.5 times lower, which proves that its installation on the detector is reasonable. However, a fast neutron background, resulting from the interaction of cosmic muons with lead nuclei, enhances inside the lead shielding. Indeed, the $5 \mathrm{~cm}$ lead shielding around a neutron detector doubles its count rate. Therefore, a layer of borated polyethylene should be placed inside the lead shielding.

After the installation of the passive shielding, we carried out detailed measurements of gamma background inside it to determine actual conditions around the detector. Figure 4 presents the gamma spectrum inside the passive shielding for various distances along the route of the detector: $6.28 \mathrm{~m}$, $8.38 \mathrm{~m}$, and $10.48 \mathrm{~m}$. No noticeable alterations in the spectrum shape were observed. Moreover, for comparison, gamma spectra are measured at the reactor on and off inside the passive shielding, at the point nearest to the reactor. A considerable difference in these spectra was not found.

\section{ESTIMATIONS OF FAST NEUTRON FLUX}

In 2013, at the SM-3 reactor, all preparations of the neutrino laboratory room were completed as well as the installation of the passive shielding of the neutrino detector.

The slide valve of the former neutron beamline was carefully plugged. As a result, a flux of thermal neutrons in the neutrino laboratory room decreased 29 times to the level of $1 \div 2 \times 10^{-3} \mathrm{n} /\left(\mathrm{cm}^{2} \mathrm{~s}\right)$. This level is an effect of cosmic rays and, practically, is independent of the reactor operation.

Measurements of thermal neutron flux were made with a ${ }^{3} \mathrm{He}$ detector, which is a proportional counter $1 \mathrm{~m}$ long with a diameter of $30 \mathrm{~mm}$. For the registration of fast neutrons, we used a same ${ }^{3} \mathrm{He}$ detector, but it was placed into a shielding made of polyethylene (thickness of layer is $5 \mathrm{~cm}$ ), which in its turn was wrapped in a layer of borated rubber (3 mm thick, containing 50\% boron). Thermal neutrons

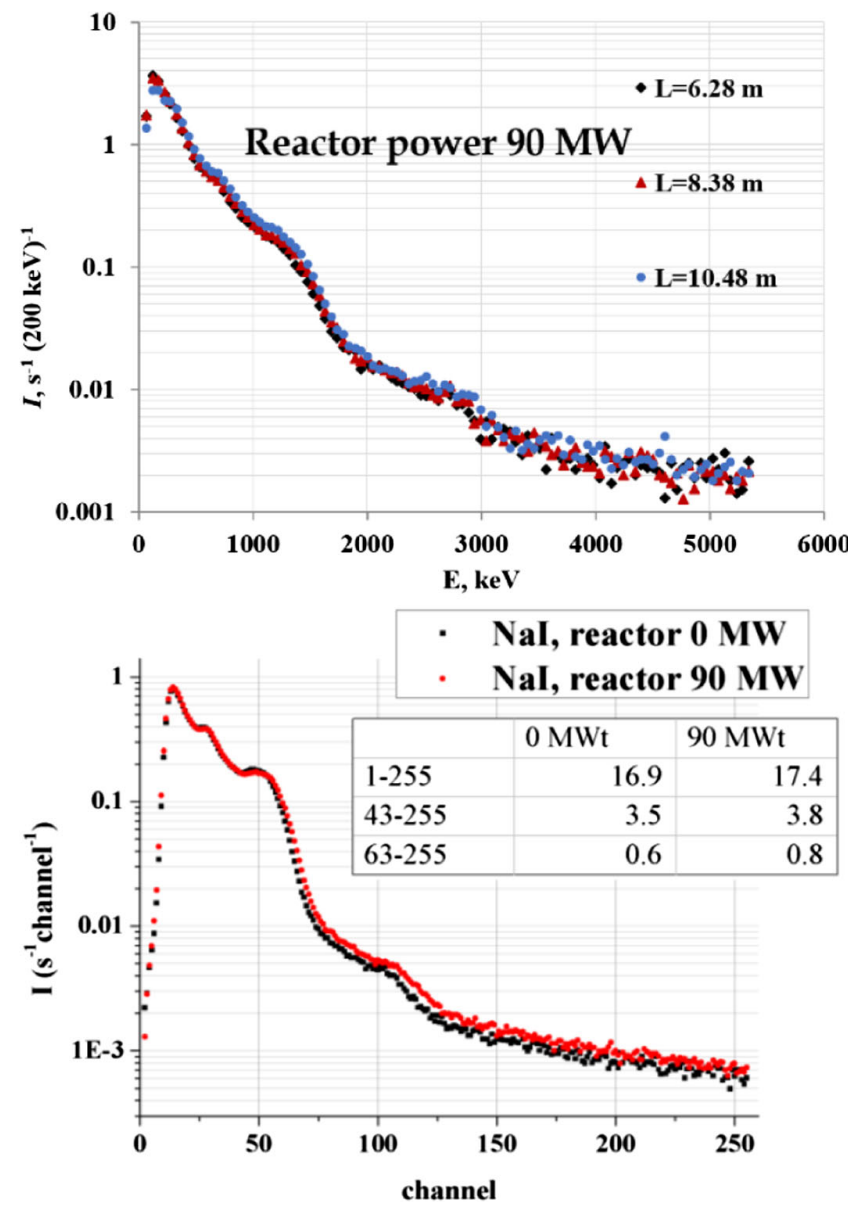

FIG. 4. Gamma spectra at the detector location. Top: reactor power is $90 \mathrm{MW}$. L is distance from the center of the reactor core: $-6.28 \mathrm{~m},-8.38 \mathrm{~m},-10.48 \mathrm{~m}$. Bottom: reactor on/off spectra.

stop in borated rubber while fast neutrons penetrate it and slow down in polyethylene to be registered by ${ }^{3} \mathrm{He}$ detector.

To convert the count rate $\left(\mathrm{s}^{-1}\right)$ of the proportional counter ${ }^{3} \mathrm{He}$ detector into neutron flux density $\left(\mathrm{cm}^{-2} \cdot \mathrm{s}^{-1}\right)$, both detectors were calibrated using a standard detector MCS AT6102. For this purpose, the ${ }^{3} \mathrm{He}$ detector and MCS were placed side by side at the distance of $3 \mathrm{~m}$ from a neutron source (Pu-Be).

${ }^{3} \mathrm{He}$ detectors of thermal and fast neutrons have a sensitivity 2 orders of magnitude higher than that of a standard MCS detector. They were employed for conducting measurements of low background. Estimations of the neutron background were made, for the first time, before upgrading former neutron beamline enter (before slide valve was plugged), then, after upgrading and, finally, after installing passive shielding of the neutrino detector.

In measurements of the fast neutron flux outside the passive shielding, the detector of fast neutrons was located on the roof of the passive shielding near the reactor wall, i.e., at a distance of $5.1 \mathrm{~m}$ from the reactor core. The results of measurements are shown in Fig. 5 on the left. The flux is 

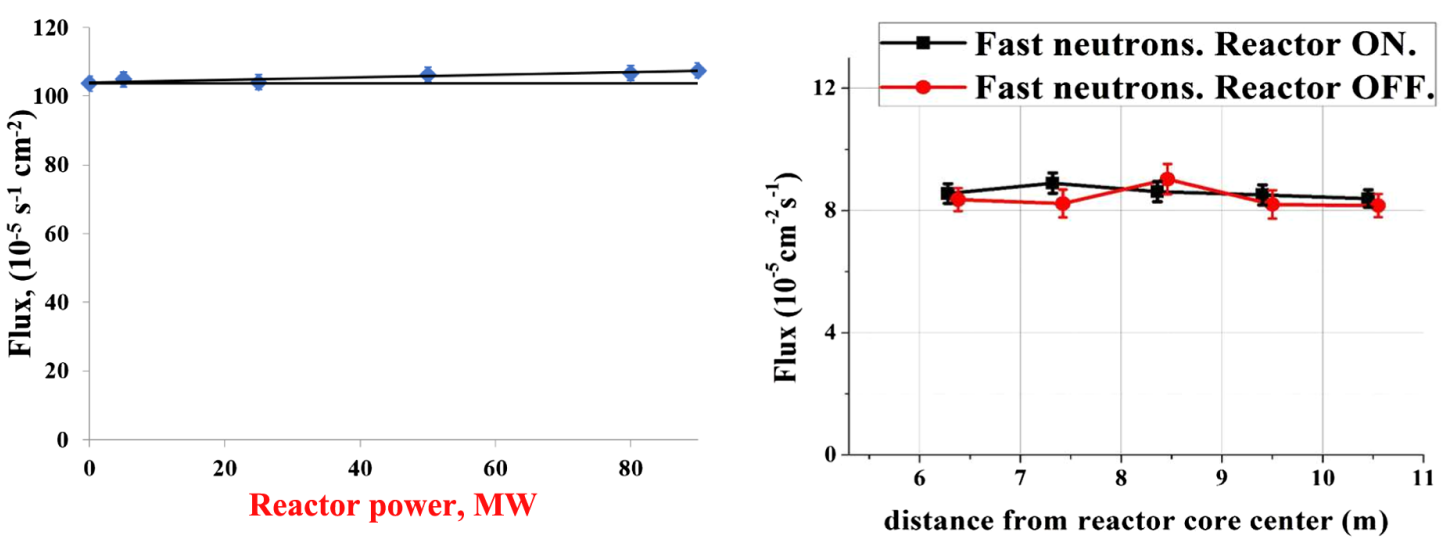

FIG. 5. Left: plot of neutron flux (near the reactor wall, i.e., at distance $5.1 \mathrm{~m}$ from the reactor core) as a function of reactor power. Right: Fast neutron background at various distances from the reactor core measured with the detector of fast neutrons inside passive shielding. The detector of fast neutrons was placed on top of the neutrino detector and was moved with it.

almost independent of the reactor power, and its value is $10^{-3} \mathrm{~s}^{-1} \mathrm{~cm}^{-2}$. The excess above the level of the cosmic background at the full reactor power is $(5 \pm 2) \%$. The measurements of the fast neutron flux inside the passive shielding were carried out in two modes. The first one was aimed at determining the influence of the reactor. For that purpose, the measurements of the fast neutron flux were performed inside the passive shielding at the position nearest to the reactor wall with an operating reactor and a reactor in off mode. Both measurements were carried out for 10 days. With the operating reactor, the fast neutron flux was equal to $(5.54 \pm 0.13) 10^{-5} \mathrm{~s}^{-1} \mathrm{~cm}^{-2}$, while with the switched off reactor, it was $(5.38 \pm 0.13) 10^{-5} \mathrm{~s}^{-1} \mathrm{~cm}^{-2}$; i.e., there was no difference within the accuracy of $2.5 \%,(0.16 \pm 0.13) 10^{-5} \mathrm{~s}^{-1} \mathrm{~cm}^{-2}$.

A more accurate estimate of the fast neutron flux from the reactor within the passive shielding can be made using a suppression factor of 12 for the fast neutron flux inside the passive shielding. Then the excess of the fast neutron flux above the cosmic background level at the full reactor power inside the passive shielding is $(0.38 \pm 0.15) \%$. Thus, at a signal/background ratio of 0.5 , the contribution of fast neutrons from the reactor to the on-off neutrino signal can be $(1.1 \pm 0.45) \%$. This is not a problem for these measurements, especially since this background cannot have the oscillation behavior.

The second mode was aimed at the direct measurement of the fast neutron background inside passive shielding on the neutrino detector route. For that purpose, the detector of fast neutrons was installed on top of the neutrino detector and was moved with it inside the neutrino beamline in a range from $6.25 \mathrm{~m}$ to $10.5 \mathrm{~m}$ from the reactor. The results of this measurements with operating and switched off reactor are shown in Fig. 5 (on the right). There is no difference caused by the reactor mode within statistical accuracy. Also, the background does not depend on distance. In these measurements, the background level appeared to be equal to $(8.5 \pm 0.1) 10^{-5} \mathrm{~s}^{-1} \mathrm{~cm}^{-2}$, which is somewhat higher than that near the reactor wall. The discrepancy can result from the detector positioning relative to the direction of a neutron flux, near the reactor wall, it was installed vertically, while on the top of the neutrino detector, it was installed horizontally.

Finally, we can conclude that the fast neutron background is almost independent of the reactor working mode, but it is determined by the neutrons created in the interaction of cosmic ray muons with matter around the detector. Notice that passive shielding contributes to the neutron background, because muons interact with the materials of the shielding. However, passive shielding suppresses the fast neutron background by a factor of 12 , so that fast neutron flux outside the passive shielding is $10^{-3} \mathrm{~s}^{-1} \mathrm{~cm}^{-2}$, while flux inside the shielded area is $(8.5 \pm 0.1) 10^{-5} \mathrm{~s}^{-1} \mathrm{~cm}^{-2}$.

\section{INVESTIGATION OF THE BACKGROUND CONDITIONS WITH ANTINEUTRINO DETECTOR MODEL}

Before the measurements with a full-scale neutrino detector, we tried the model of it. The model of the antineutrino detector contains 4001 of liquid scintillator BC-525 with addition of $\mathrm{Gd}$ with a concentration $1 \mathrm{~g} / \mathrm{l}$, 16 PMTs on the top, and five plates of active shielding (muon veto) around it. The model of the antineutrino detector and active shielding installed in the passive shielding is shown in Fig. 6.

Figure 7 presents the spectrum obtained with the antineutrino detector model, which can be divided into four parts. The first part (up to $2 \mathrm{MeV}$ ) corresponds to the background radioactive contamination; the second part (from $2 \mathrm{MeV}$ to $10 \mathrm{MeV}$ ) covers the neutron/neutrino registration area, since it corresponds to the energy of gammas emitted in neutron capture by Gd. The range from 10 to $60 \mathrm{MeV}$ is related to the soft component of cosmic rays which is a result of muon decays and muon captures in 


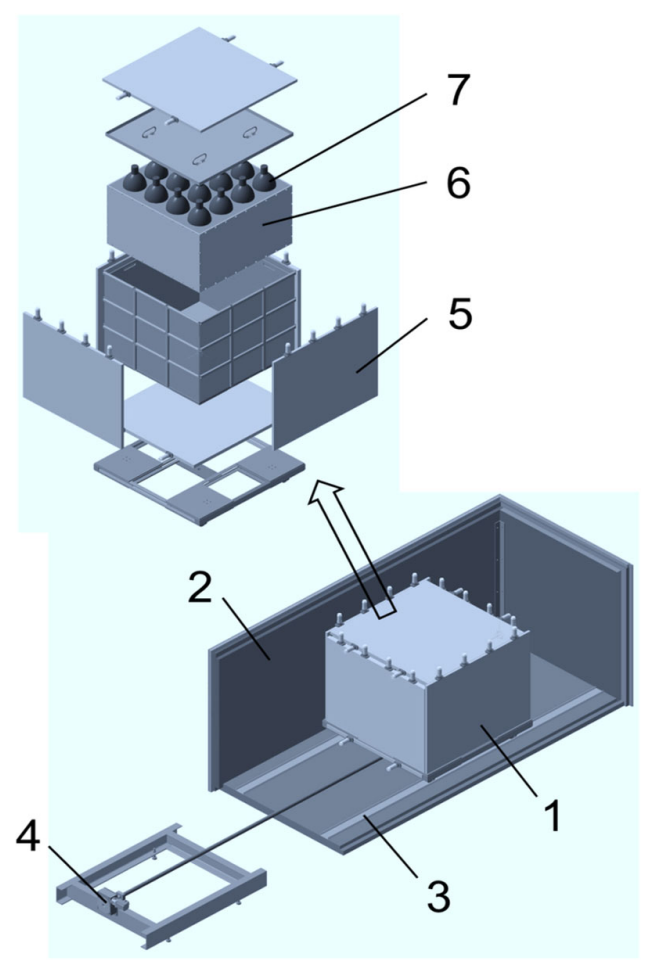

FIG. 6. Model of the neutrino detector installed in the passive shielding [25,26]. 1-detector of reactor antineutrino, 2-passive shielding, 3-rails, 4-engine for detector movement, 5-active shielding with PMT, 6-volume wit liquid scintillator with Gd, 7-detector's PMT.

matter. And finally, the range $60-120 \mathrm{MeV}$ is related to muons passing through the detector. This picture also illustrates small alterations of the spectrum shape for different detector positions.

In the course of long-term measurements [25,26], temporal variations of cosmic ray intensity have been found. They are caused by fluctuations of atmospheric

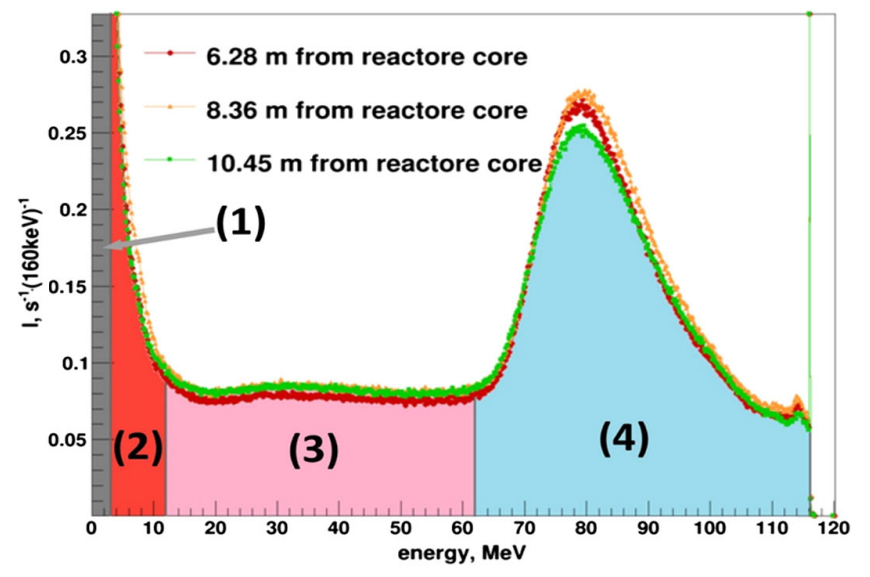

FIG. 7. Detector energy spectrum at different distances from the reactor core and a division of spectrum into zones: 1 -radioactive contamination background, 2-neutrons, 3-soft component of cosmic rays, 4-muons.

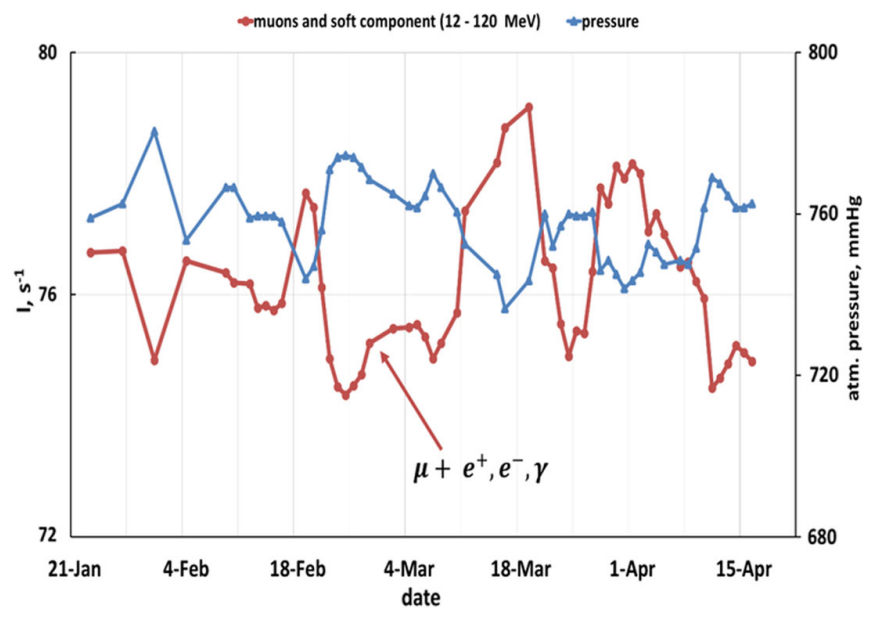

FIG. 8. Barometric effect of cosmic rays: the left axis illustrates a summary detector count rate in the energy areas 3 and 4, the right axis shows atmospheric pressure, the horizontal axis gives the measurement time since 23 of January till 15 of April in 2014.

pressure and season changes of temperature. These are well-known barometric and temperature effects of cosmic rays (Figs. 8 and 9).

The behavior of fast and slow components differs by additional long-term drift, with the drift sign being opposite for fast and slow components. It is the so-called temperature effect, which can be interpreted in the following way. At the rise of the temperature in lower atmospheric layers, their expansion results in the increase of the average altitude of the creation of muon fluxes. As the distance to the Earth grows, the share of the decayed muons is getting larger. Thus, the intensity of the fast component (muons) decreases and that of slow component (products of decay: electrons, positrons, gamma quanta) rises.

Figure 9 illustrates the drift effect with opposite signs for fast and slow components of cosmic background with increasing temperature of the lower layers of atmosphere in the vicinity of the Earth surface from January till April: from $-30 \mathrm{C}$ to $+10 \mathrm{C}$.

The fluctuations in the cosmic background are determined by the fluctuations in atmospheric pressure that is about $\pm 1.1 \%$. From the studies of variation in the cosmic background, an important quantitative conclusion can be made about their effect on measurements.

The direct results of variation in the cosmic background of the statistical distribution of the neutrino signal will be presented in Sec. XII in Fig. 31.

\section{COSMIC BACKGROUND, ACTIVE SHIELDING. ENERGY AND TIME SPECTRA OF CORRELATED SIGNALS}

The antineutrino flux is measured with an inverse betadecay reaction $\bar{\nu}_{e}+p \rightarrow e^{+}+n$. For the registration of these signals, we use the delayed coincidence method. 


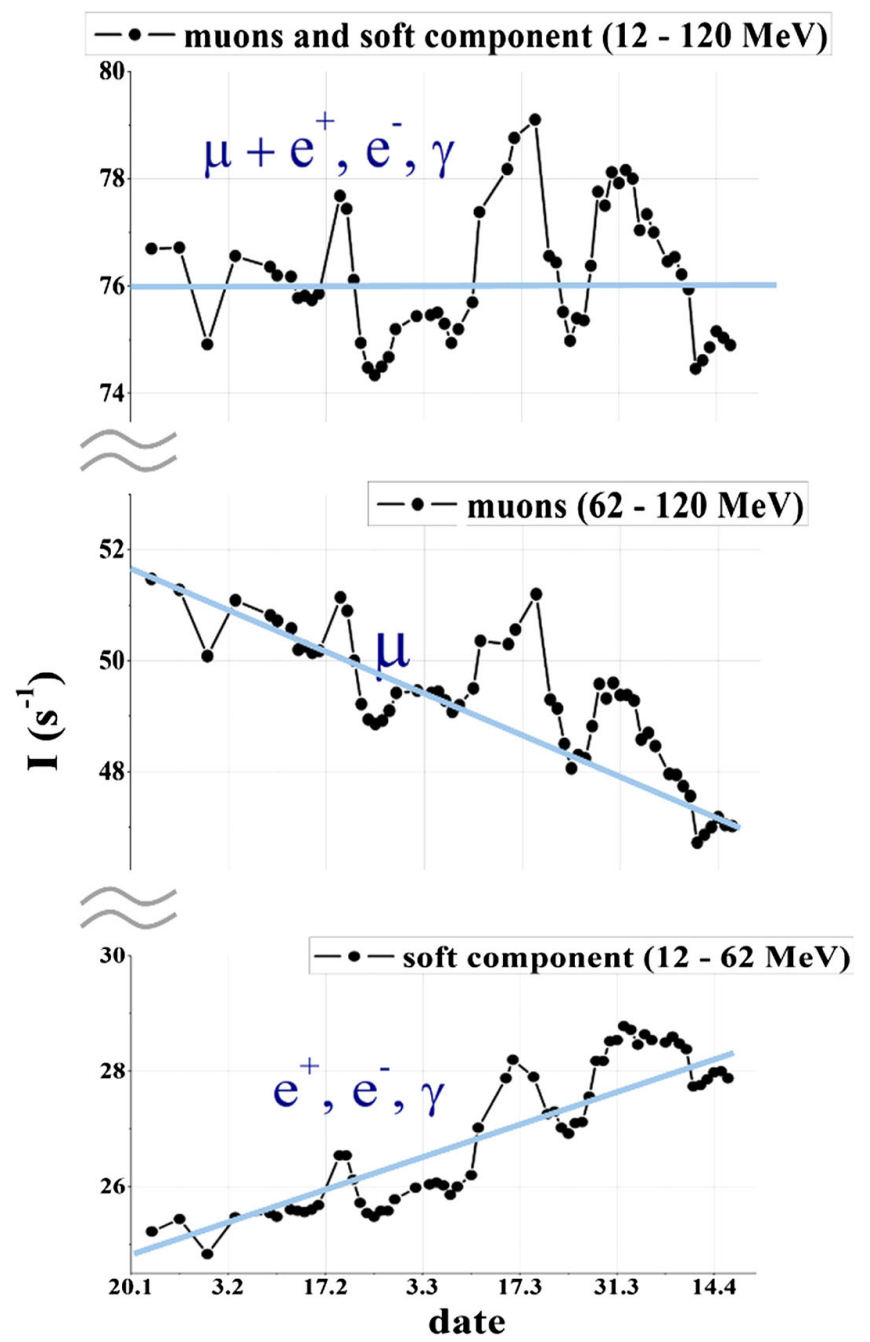

FIG. 9. The barometric and temperature effects of cosmic rays: top-a summary detector count rate in the energy areas 3 and 4, middle - a detector count rate in the energy area 4, bottom-a detector count rate in the energy area 3 . The horizontal axis is the measurement time since 23 January till 15 April of 2014.

In that measurement scheme, an event starts with a registration of a signal with suitable characteristics. It opens a time window in which we expect to register a delayed signal with another set of suitable parameters. In the end of the time window when the probability of neutron capture is very low, the background of accidental coincidence is measured. The measured value of the accidental coincidence background can be subtracted from the results.

In our experiment, the correlated background is caused by cosmic ray muons. Therefore, when the reactor is switched off, our detector and the method of delayed coincidence can be used to measure muon background. Muons in the detector create a delayed signal if either muon stops in the scintillator creating a muon atom where this muon decays with lifetime of $2.2 \mu$ s or muon interaction with matter inside the detector results in emission of an evaporation neutron, which is captured by gadolinium after slowing down for $5 \mu \mathrm{s}$. The characteristic time of muon capture by $\mathrm{Gd}$ in scintillator with Gd concentration of $0.1 \%$ is $31.3 \mu \mathrm{s}$.

Figure 10 illustrates the time spectra of delayed coincidences. The background of accidental coincidences is subtracted. The upper black curve represents measurements without using the active shielding of the detector. One can see two exponents (straight lines in logarithmic scale), which correspond to a muon decay and a neutron capture by Gd. The integral under the first exponent corresponds to the stopped muons count rate of $1.54 \mu / \mathrm{s}$, and the slope corresponds to a muon lifetime $(2.2 \mu \mathrm{s})$. The integral under the second exponent corresponds to a neutron capture rate in the detector $-15 \mathrm{~s}^{-1}$, and the parameter of the exponent $(31.3 \mu \mathrm{s})$ corresponds to the neutron lifetime in the scintillator with $0.1 \%$ of $\mathrm{Gd}$.

The number of muon stops per second agrees with the estimation based on the data about muon flux and the scintillator mass, while neutron capture rate agrees with the calculated rate of neutrons generated in the detector itself, as a result of the muon flux passing through it. Adequacy of the installed passive shielding is confirmed by the fact that an additional $10 \mathrm{~cm}$ of borated polyethylene above the neutrino detector do not change fast neutron count rate.

The muon background can be significantly suppressed by employing of active shielding of the detector and rejecting detector signals with too high energy. If the system gets the signal from active shielding or if measured energy in the detector exceeds $9 \mathrm{MeV}$ then the system stops registering signals for time $100 \mu \mathrm{s}$.

One of the major problems in our experiment is to separate correlated events from background of accidental coincidences. An example of measurements of a spectrum of delayed signals is shown in Fig. 11. The lifetime of neutron in the scintillator with $\mathrm{Gd}$ is $31.3 \mu \mathrm{s}$, so a neutron will be captured in $200 \mu$ s after the prompt signal with a probability higher than $99 \%$. Assuming that the background of accidental coincidences has a uniform distribution in time, we can use an interval after $200 \mu$ s to measure it. So, measuring the rate of delayed coincidences, we also control rate of background of accidental coincidences at the same time. To measure the background, we chose an interval of $100 \mu$ s so the total time window in which we expect a delayed signal is $300 \mu \mathrm{s}$.

Besides the time in which a delayed signal occurs, we have another parameter to select neutrino events the energies of prompt and delayed signals. When we determine exact energy region in which we search for signals, we try to obtain the best signal to background ratio. A positron signal has a natural threshold of $1 \mathrm{MeV}$ of the total energy of its annihilation. Therefore, the lower limit of the registered energy range is higher than $1 \mathrm{MeV}$. The lower limit is the more antineutrino events we register, but at the same time, the higher is background of accidental 


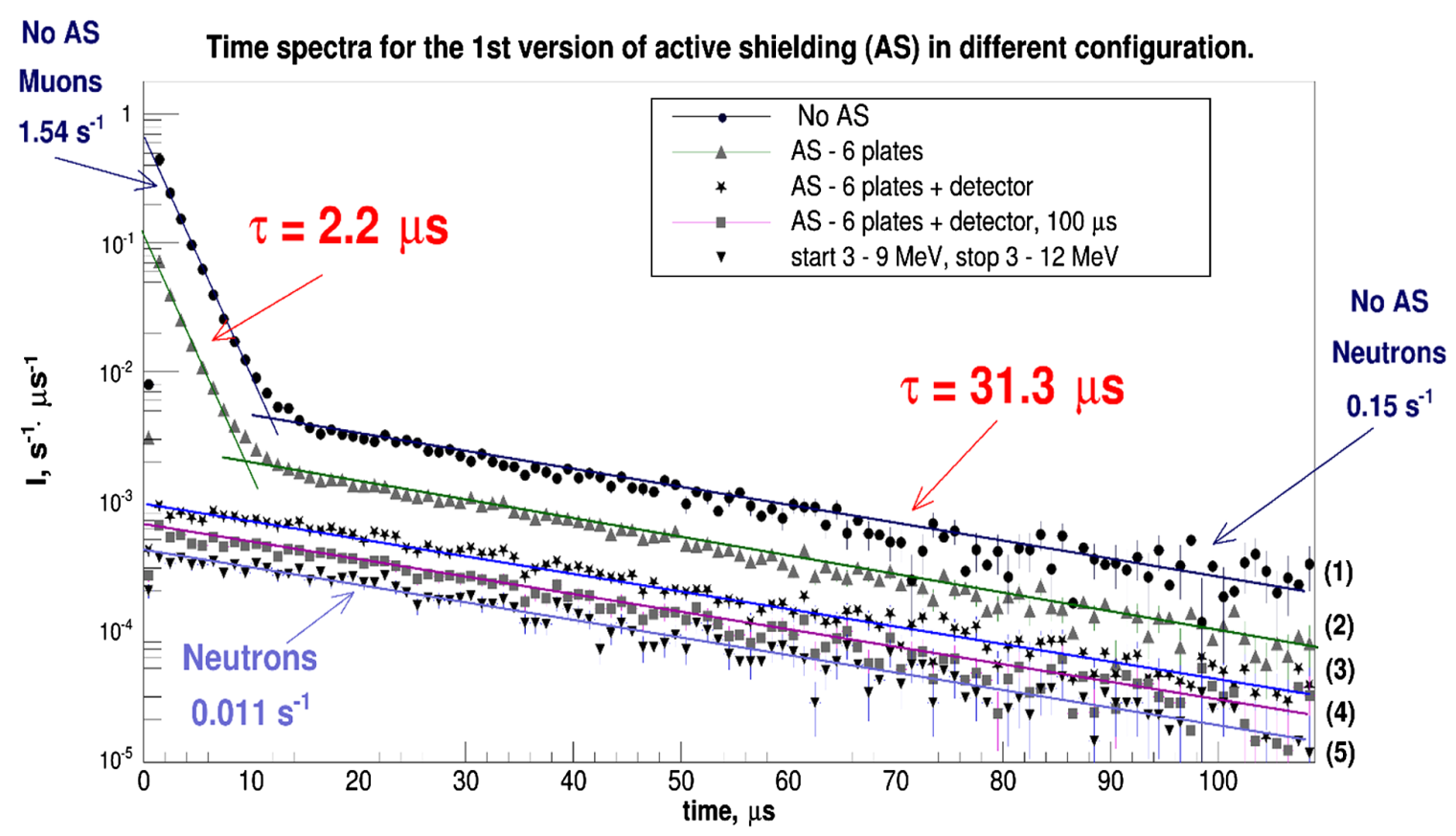

FIG. 10. Time spectra at different configurations of active shielding: 1-no active shielding, 2-plates of active shielding are using, 3 - the same + ban from the detector at signals higher than $12 \mathrm{MeV}, 4$ - the same + ban on $100 \mu$ s after the detector signal, at energy higher than $12 \mathrm{MeV}$, or after the signal in active shielding, 5- the same + limit on start and stop signals in ranges 3-9 MeV and 3-12 MeV, respectively.

coincidences. Time and energy spectrum of delayed signals obtained with threshold $3 \mathrm{MeV}$ are shown in Fig. 11.

The background of accidental coincidences is sufficiently low while the amount of antineutrino events is at the acceptable level. The point is a signal of neutron capture by Gd that has a sufficient amount of energy - up to $8 \mathrm{Mev}$, while background of natural radioactivity is negligible on energy region above $3 \mathrm{MeV}$. In decreasing the lower threshold from $3 \mathrm{MeV}$ to $1.5 \mathrm{MeV}$, the rate of accidental coincidences considerably increases (Fig. 12).

The upper energy limit is determined by a spectrum of the reactor antineutrino and emission of energy in neutron capture by $\mathrm{Gd}$. The lower limit in the neutrino signal registration must be $1 \mathrm{MeV}$. The study of the influence of energy limits on background and efficiency of antineutrino registration was concluded in setting ranges for prompt and
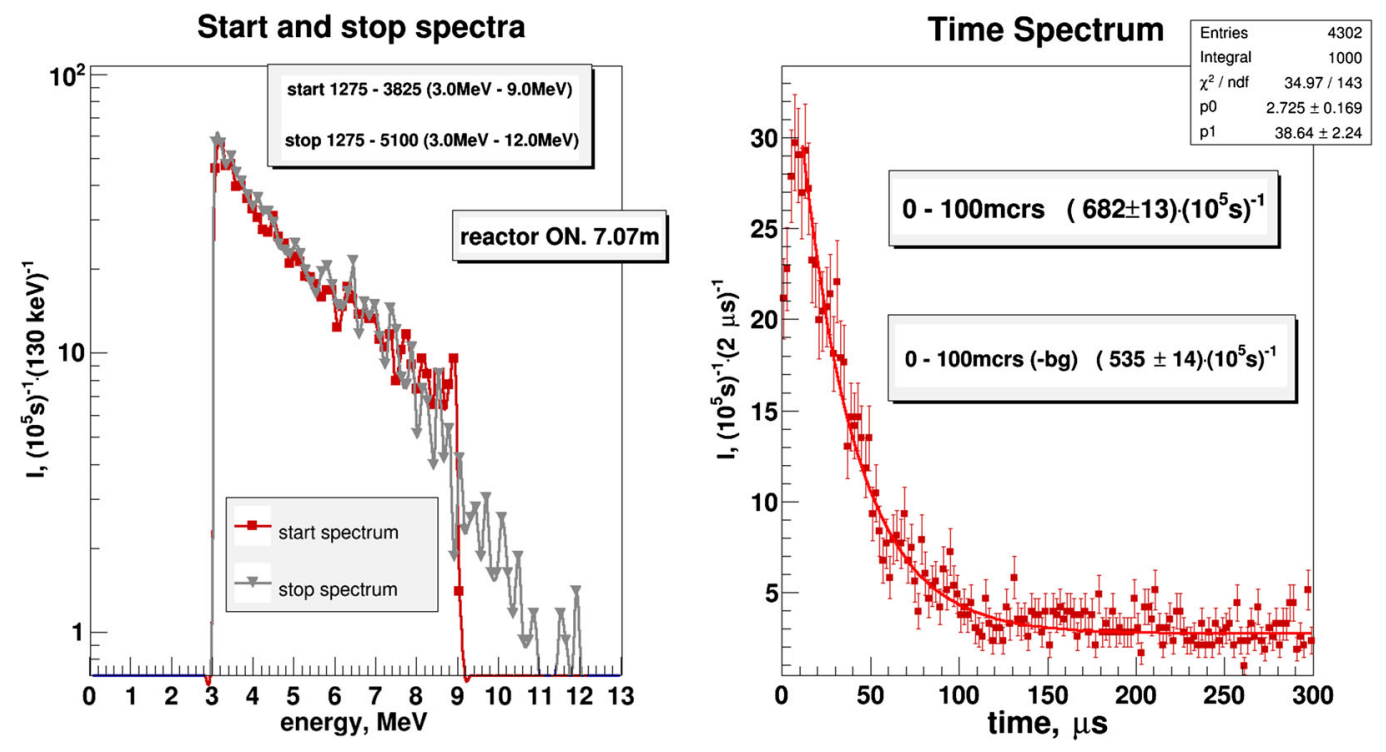

FIG. 11. Energy spectra of prompt and delayed signals (left) and time spectra (right): threshold of start and stop signals 3-9 MeV and 3-12 MeV, correspondingly. 

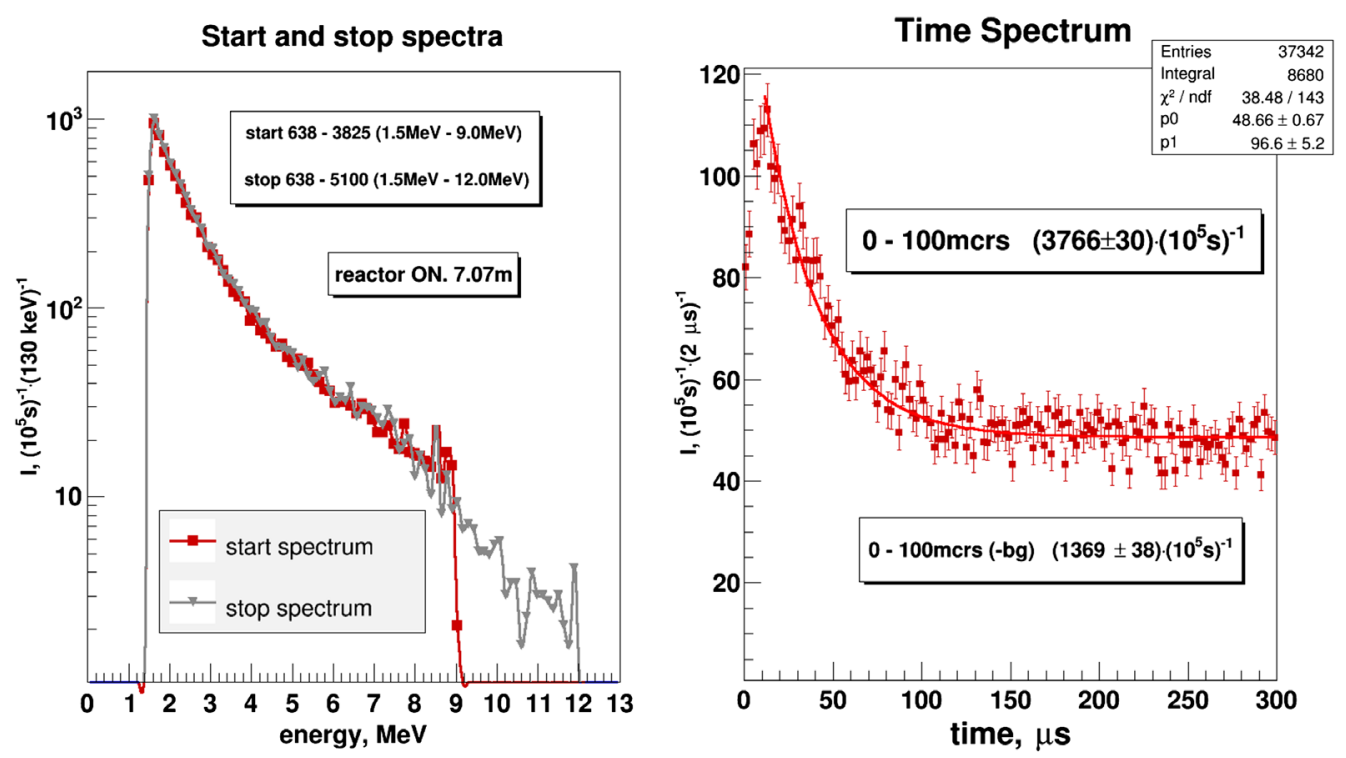

FIG. 12. Energy spectra of prompt and delayed signals and time spectra: $1.5-9 \mathrm{MeV}$ and $1.5-12 \mathrm{MeV}$.

delayed signals $1.5-9 \mathrm{MeV}$ and 3-12 MeV, correspondingly. Using the active shielding veto and selecting signals by energy, we managed to suppress the background of correlated signals caused by neutrons to the level of $1.1 \times 10^{-2} \mathrm{~s}^{-1}$. We assume that the remaining background is caused by fast neutrons, which appears in interactions of cosmic muons with matter outside the detector. In that case, the prompt signal is created by a recoil proton. That background cannot be suppressed by using the active shielding because it does not register fast neutrons. The background of fast neutrons emitted outside the detector appears to be the main problem of our experiment and the solution of this problem is described in the next section.

\section{CARRYING OUT RESEARCH WITH A MODEL OF ANTINEUTRINO DETECTOR OF A MULTI-SECTION TYPE}

The first measurements carried out with the detector model revealed that the combination of the active and passive shielding and selection of events by energy are yet not enough to suppress the correlated background. After suppressing other sources of background events, fast neutrons emitted outside the detector in interactions of high energy cosmic ray muons with matter around the detector become the main source of the background.

The scattering of fast neutrons easily imitates an IBD process, which we use to detect neutrinos. Registration of the first (start or prompt) signal from recoil protons imitates the registration of a positron. The second (stop of delayed) signal arises in both cases when a neutron is captured by gadolinium. The active shielding cannot help to distinguish fast neutron signals from antineutrino signals (Fig. 13).

A multisection scheme was developed to get additional selection criteria for antineutrino events. There is a difference in the localization of prompt signals of antineutrino and neutron events. A recoil proton in matter has a track of about $1 \mathrm{~mm}$ length, while a positron emitted in an IBD process annihilates with the emission of two gamma quanta, each having an energy of $511 \mathrm{keV}$ and opposite directions. As a result, if the vessel with the scintillator is divided in several sections of the same size with walls reflecting optical photons, then the track of recoil proton will be contained within one section. The track of a positron has an average length of about $5 \mathrm{~cm}$, so its signal is also registered in one section, but gammas with an energy of $511 \mathrm{keV}$ can be registered in adjacent sections.

The detector inner vessel was divided into 16 sections $0.225 \times 0.225 \times 0.5 \mathrm{~m}^{3}$ with rigidly fixed partitions between them. At the same time, we started to use the active shielding consisting of two layers external and

\section{The problem of fast neutrons}

\section{False event}

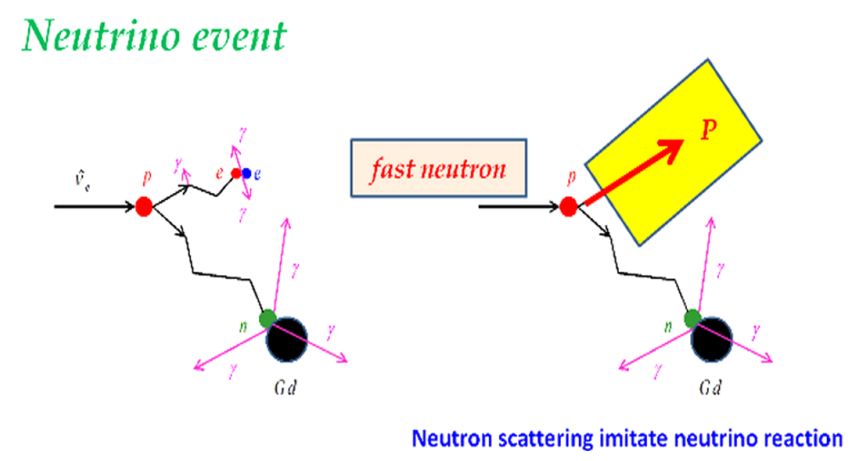

FIG. 13. Illustration of the background problem caused by the fast neutrons. 


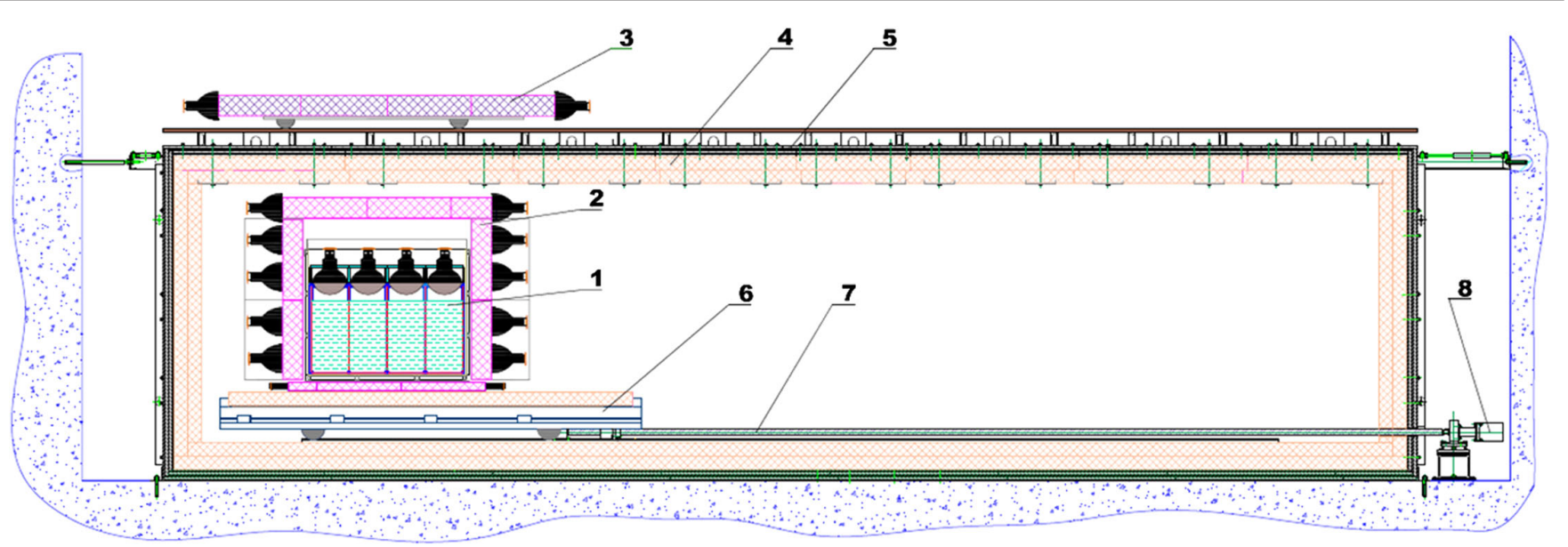

FIG. 14. General scheme of an experimental setup: 1-detector of reactor antineutrino, 2-internal active shielding, 3-external active shielding (umbrella), 4-borated polyethylene passive shielding, 5—-steel and lead passive shielding, 6-moveable platform, 7-feed screw, 8-step motor.

internal relative to passive shielding. The external layer ("umbrella") moves on the roof of the passive following the detector movements [27]. The scheme of locations of the multisection detector and active shielding relative to the passive shielding is shown in Fig. 14.

The ratio of single-section and multisection prompt signals of antineutrino events depend on the detector configuration, amount of sections, and their sizes.

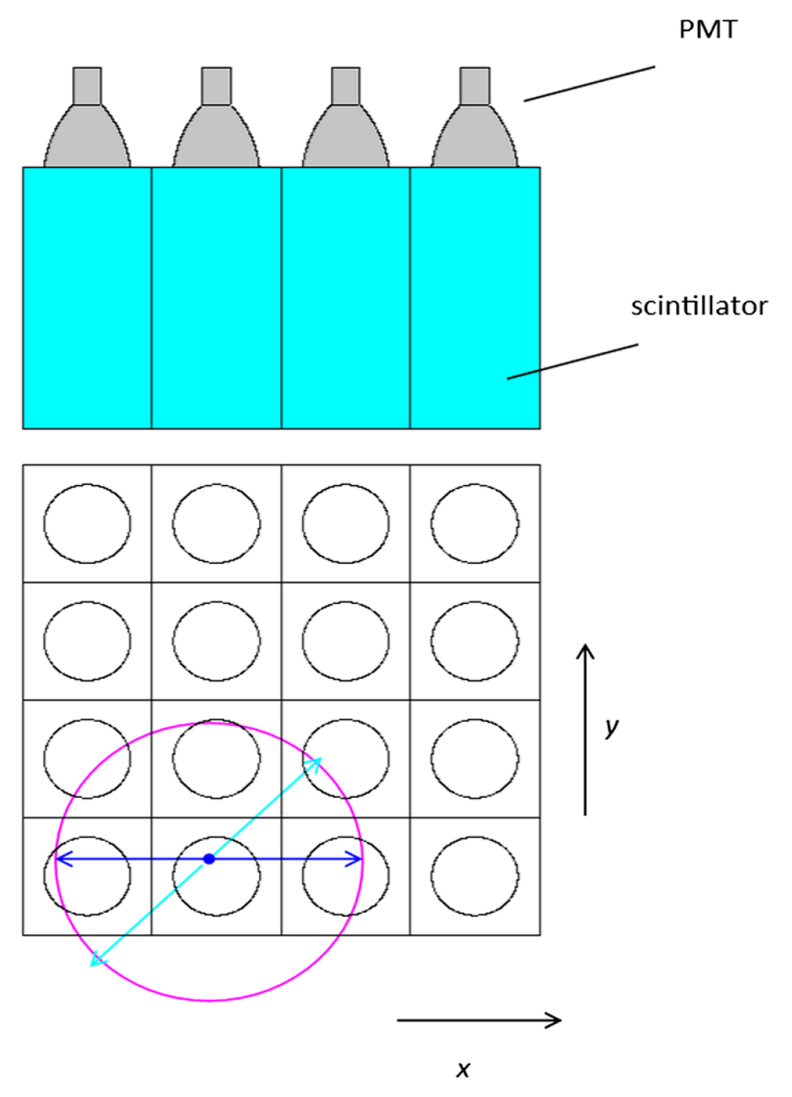

FIG. 15. Scheme of the detector of reactor antineutrinos.
Therefore, a Monte Carlo simulation of a particular detector configuration is required.

The detector scheme for the Monte Carlo calculation is presented in Fig. 15. The probability of recording double starts depends on the section location: in the center, on the sides, or in the corner. The probability of the registration of double starts for different sections where the event occurred is presented in Table I.

The mean probability of double starts over all the detector is $30 \%$. That means in our method $70 \%$ of prompt signals of antineutrino events occurs in a single section. Therefore, if we only consider events with double starts then the number of registered events decrease by 3 times, which is obviously unacceptable. But the analysis of results can be performed by using the single-section and multisection events, and use their ratio to the total amount of the events (30\% and $70 \%)$ as an additional criterion that can be used to check the validity of the selection of antineutrino events. Thus, if the signal difference between the reactor on and reactor off measurements is within $30 \%-70 \%$ for the multisection and single-section events, then it can be interpreted as a neutrino signal.

Preliminary measurements with the $\mathrm{Pu}-\mathrm{Be}$ fast neutron source have been made before the start of measurements with the new multisection detector model. The time spectra of the single-section and multisection prompt signals are shown in Fig. 16. It appears that if we consider only multisection prompt signals, then the correlated signals from neutrons are completely excluded, and only a straight line from an accidental coincidence remains.

TABLE I. Probability of registration of double starts.

\begin{tabular}{lccc}
\hline \hline Central cell & Side cell & Angular cell & In all cells \\
\hline 0.424 & 0.294 & 0.188 & 0.300 \\
\hline \hline
\end{tabular}




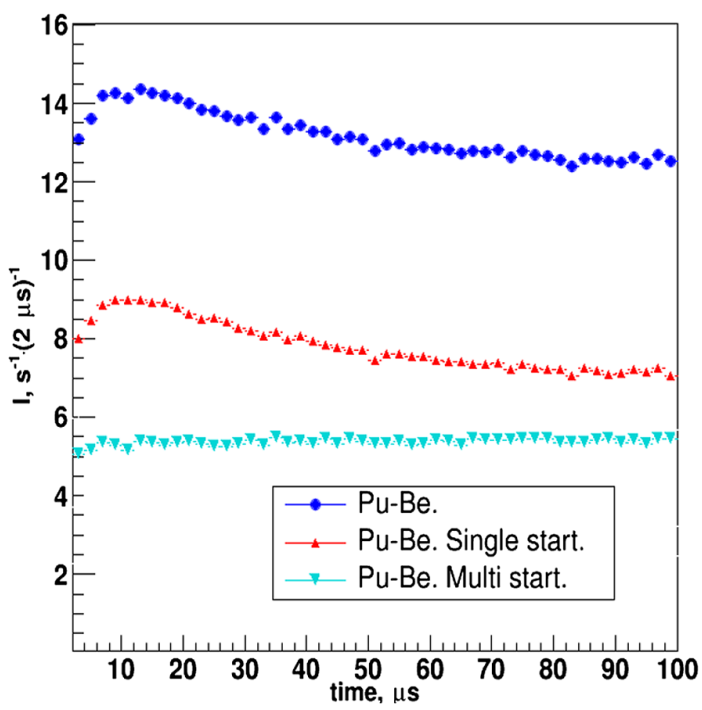

FIG. 16. Time spectra of the delayed coincidence obtained with a fast neutron source. The red curve corresponds to single section starts, and the green one shows multisection starts.

This experiment has revealed that fast neutrons give only single-section starts.

The count rate difference (on-off, i.e., with the reactor switched on and off) for two-section and single-section starts, integrated over all distances, makes up $(29 \pm 7) \%$ and $(71 \pm 13) \%$, respectively. Within the available accuracy, such a ratio allows us to assume the registered events as neutrinolike events. Thus, it is another evidence that the on-off signal corresponds to neutrino events.

However, the accuracy of this statement is lower than direct estimates of the contribution of fast neutrons from the reactor to the on-off neutrino signal, which can be $(1.1+0.45) \%$ as shown in Sec. V.

Unfortunately, we cannot use the two-section starts selection since we lose significantly in data collection.

In order to determine the energy resolution of the detector, a single section detector was manufactured to carry out detailed research. We use the effect of total internal reflection of the light from the border between scintillator and air at low angles to align the collection of light (make it homogeneous) from various distances. The problem is that in the case of optical contact of the scintillator and PMT, the light is better collected from the distances close to the PMT in a solid angle close to $2 \pi$. The light from farther positions comes through the mirror light guide, while efficiency of light transportation for angles close to the right angle is much worse because of multiple refractions. The effect of total internal reflection at the border of scintillator and air at low angles of descent evens the transportation conditions for light coming from various distances. Finally, a mirror at the bottom of the light guide also helps to even light collection conditions for various positions in the detector section. Demonstration of the effects described above is shown in Fig. 17 on top.
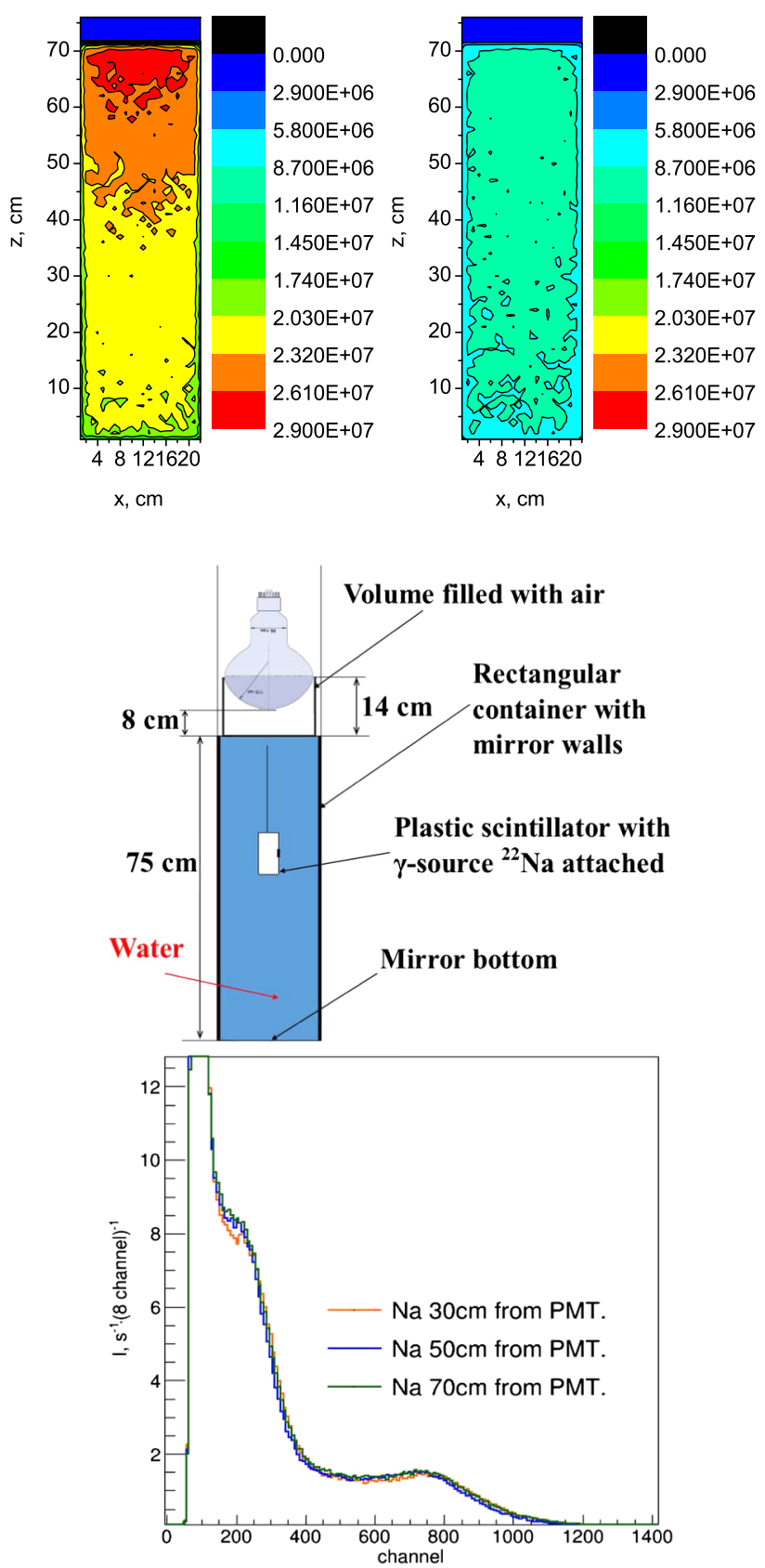

FIG. 17. Top: results of MC simulation for model of single section in case with optical contact (on the left) and without optical contact (on the right). Middle: scheme of model to measure with full-scale detector section analogue. Bottom: ${ }^{22} \mathrm{Na}$ source spectrum with different scintillator position for model of full-scale detector section with air gap.

The scheme of the experiment with a single section is shown in Fig. 17 in the middle. To carry out research of the efficiency of light collection with the usage of the total internal reflection, the section was filled with water, which has the refraction index close to the index of the scintillator. To simulate scintillation, we used a source made of a plastic scintillator and a gamma source ${ }^{22} \mathrm{Na}$ with lines $511 \mathrm{keV}$ and $1274 \mathrm{keV}$. The location of scintillation was determined 
by location of the source. As one can see in Fig. 17 (bottom), the gamma lines are almost independent of the location of the source $30 \mathrm{~cm}, 50 \mathrm{~cm}$, and $70 \mathrm{~cm}$ from the water surface. Therefore, the calibration of the detector with a scintillator can be carried out with a source outside the detector. That fact is very convenient for performing the calibration procedure.

\section{THE FULL-SCALE ANTINEUTRINO DETECTOR}

The model of the detector was replaced with the fullscale detector in 2016. This detector is also filled with liquid scintillator with a gadolinium concentration of $0.1 \%$. The detector inner vessel is divided into 50 sections- ten rows with five sections in each having size of $0.225 \times$ $0.225 \times 0.85 \mathrm{~m}^{3}$, filled with scintillator to the height of $70 \mathrm{~cm}$. The total volume of the scintillator is $1.8 \mathrm{~m}^{3}$. The detector is placed into the passive shielding. The neutrino detector active shielding consists of external and internal parts relative to passive shielding. The internal active shielding is located on the top of the detector and under it. The scheme of the detector and shielding is shown in Fig. 18.

The first and last detector rows were also used as an active shielding and at the same time, as a passive shielding from the fast neutrons. Thus, the fiducial volume of the scintillator is $1.42 \mathrm{~m}^{3}$. For carrying out measurements, the detector has been moved to various positions at the distances divisible by section size. As a result, different sections can be placed at the same coordinates with respect to the reactor except for the edges at closest and farthest positions.

Monte Carlo calculations have shown that $63 \%$ of prompt signals from neutrino events are recorded within one section, and only $37 \%$ of events create signal in an adjacent section [27]. In our measurements, the signal difference at the reactor on and off has a ratio of double and single prompt events integrated over all distances $(37 \pm 4) \%$ and $(63 \pm 7) \%$. This ratio allows us to interpret the recorded events as neutrino events within the current experimental accuracy. Unfortunately, a more detailed analysis of that ratio cannot be performed due to low statistical accuracy. Yet, it should be noted that the measurements of fast neutrons and gamma fluxes dependence on distance and reactor power were made before installing the detector into passive shielding (Sec. V and $[25,26])$. The absence of the noticeable dependence of the background on both distance and reactor power was observed. As a result, we consider that difference in reactor on/off signals appears mostly due to antineutrino flux from the operating reactor. That hypothesis is confirmed by the given above ratio of single and multisection prompt signals typical, especially for neutrino events.

The measurements of fast neutrons and gamma fluxes in dependence on the distance and reactor power were made before installing the detector into passive shielding. Absence of noticeable dependence of the background on both the distance and reactor power was observed. As a result, we consider that the difference in signals (reactor on-reactor off) appears mostly due to antineutrino flux from operating reactor. The signal generated by fast neutrons from the reactor does not exceed 3\% of the neutrino signal. The fast neutron background is formed by cosmic rays. The averaged over distance ratio of on-off (antineutrino) signals to background is 0.5 .

\section{ENERGY CALIBRATION OF THE DETECTOR}

Properties of one section were investigated earlier in Sec. VIII. It was revealed that the energy resolution is independent of the position of event registration. Therefore, detector calibration can be performed with a source outside the detector on top of it (see Fig. 19).

Energy calibration of the detector was performed with a $\gamma$-quanta source and neutron source $\left({ }^{22} \mathrm{Na}\right.$ by lines $511 \mathrm{keV}$

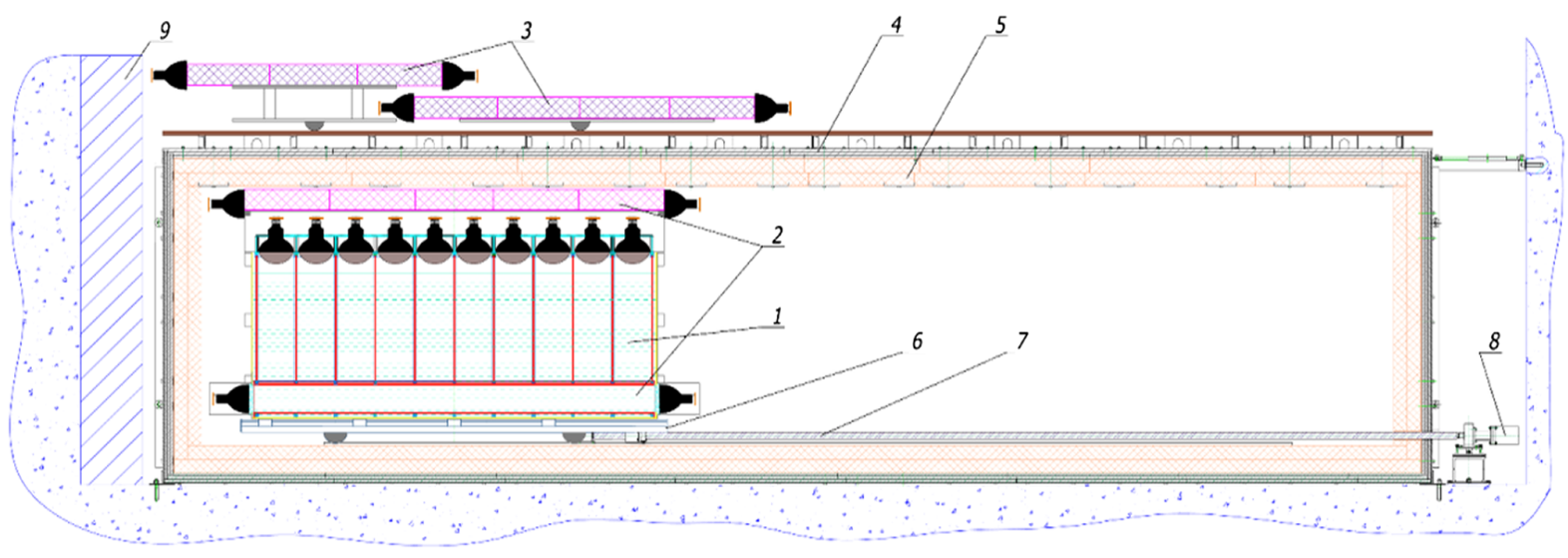

FIG. 18. General scheme of an experimental setup. 1-detector of reactor antineutrino, 2-internal active shielding, 3-external active shielding (umbrella), 4-steel and lead passive shielding, 5-borated polyethylene passive shielding, 6-moveable platform, 7-feed screw, 8-step motor, 9-shielding against fast neutrons made of iron shot. 


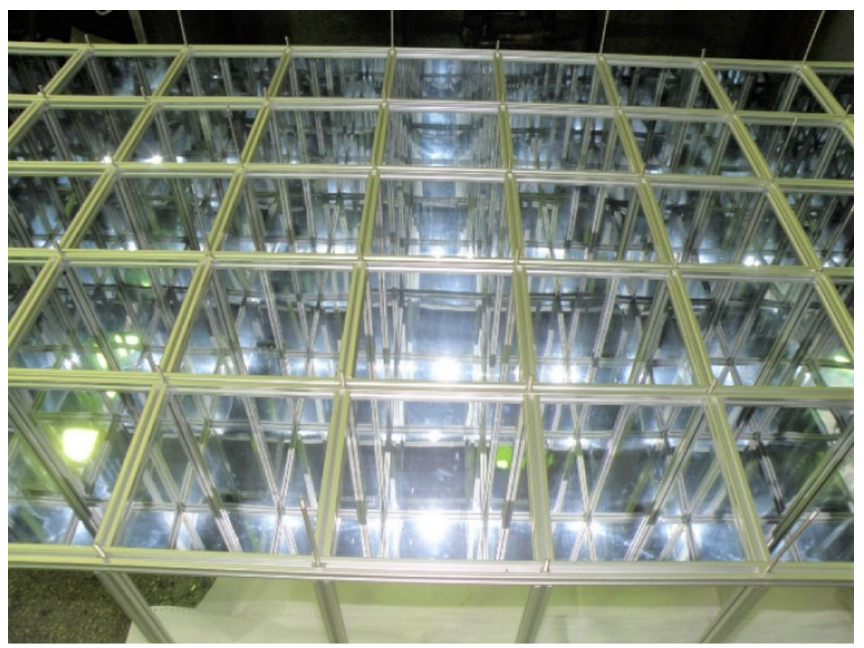

Pu-Be neutron source

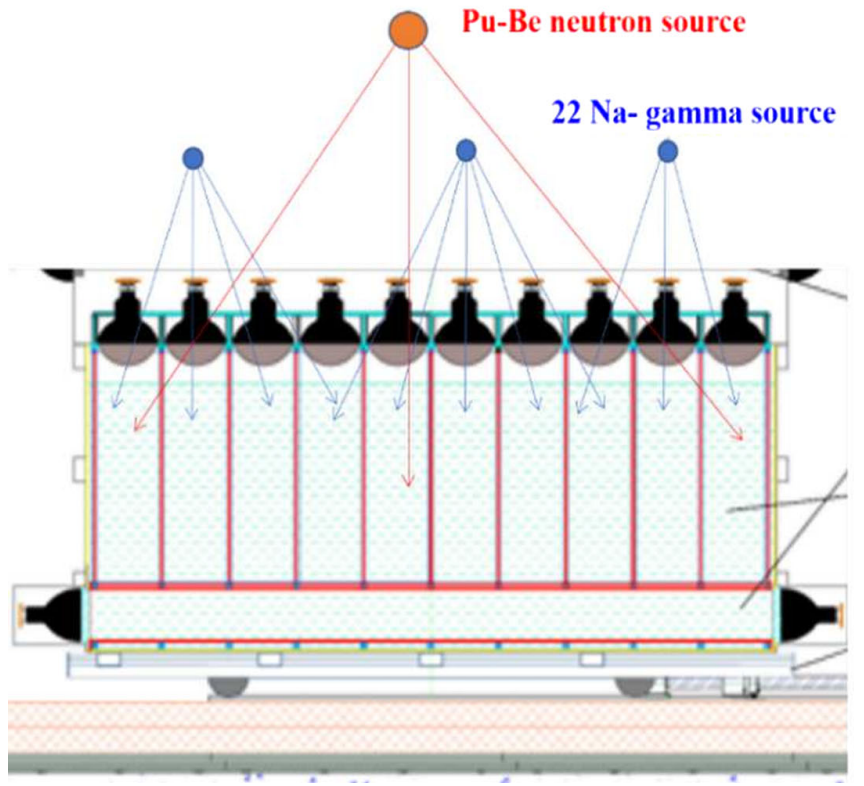

FIG. 19. The structure of the detector (top). Scheme of detector energy calibration (bottom).

and $1274 \mathrm{keV}$, by line $2.2 \mathrm{MeV}$ from the reaction $\mathrm{np}-\mathrm{d} \gamma$, by the gamma line $4.44 \mathrm{MeV}$ from $\mathrm{Pb}-\mathrm{Be}$ source, and also by the total energy of gamma quanta $8 \mathrm{MeV}$ from neutron capture in Gd) [28]. These calibration spectra are shown in Fig. 20 and more detailed in Figs. 21 and 22.

Figure 23 demonstrates linearity of calibration dependence. As a result, the spectrum of prompt signals registered by detector was measured. Its connection with antineutrino energy is determined by the equation: $\mathrm{E}_{\text {promt }}=\mathrm{E}_{\bar{\nu}}-1.8 \mathrm{MeV}+2 \cdot 0.511 \mathrm{MeV}$, where $\mathrm{E}_{\bar{\nu}}$ is antineutrino energy, $1.8 \mathrm{MeV}$ - energy threshold of IBD, and $2 \cdot 0.511 \mathrm{MeV}$ corresponds to annihilation energy of a positron.

The calibration above $2 \mathrm{MeV}$ is a problem because of the absence of required gamma sources in this energy region. Therefore, the energy resolution in Fig. 23 is extrapolated assuming a square root dependence from

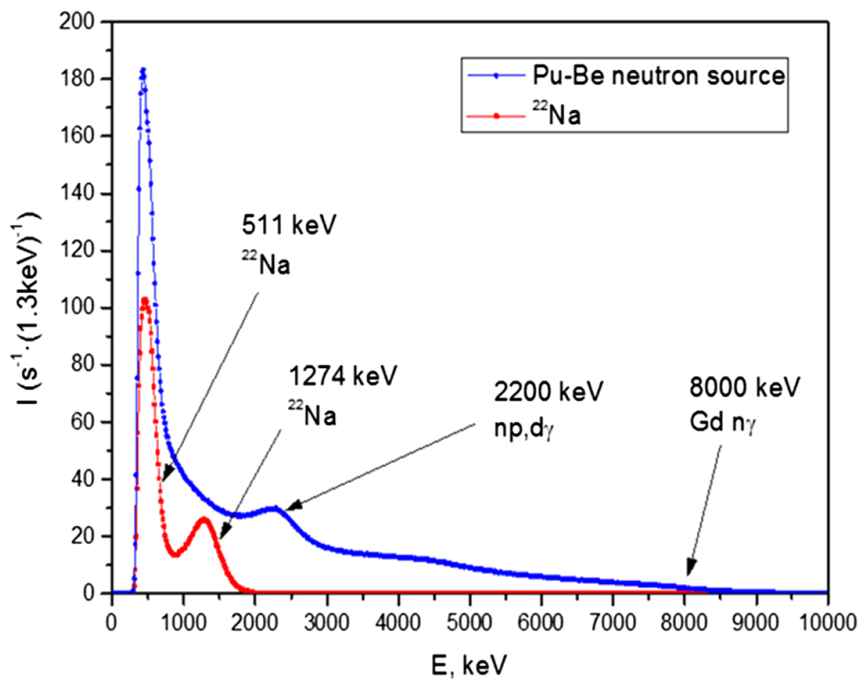

FIG. 20. The results of detector calibration.

energy. The calibration demonstrated above and the energy resolution correspond to a single section.

Actually, we are interested in the energy resolution of the detector that consisted of several sections. When a signal in a section is registered, signals from closest sections are considered too. Therefore, energy resolution of the detector is better than the energy resolution of one separate section.

The registration of a positron is a more complicated process than gamma-quanta registration. The track of a positron has an average length of about $5 \mathrm{~cm}$, so its signal is likely to be registered in one section, while gamma quanta often leave the section still having a significant amount of energy. Therefore, positron detection could be with a higher energy resolution than the detection of gamma quanta. Unfortunately, positron registration is accompanied with the emission of two gammas with energies $511 \mathrm{keV}$, which can be registered in adjacent sections. It is important to notice that registration of the gammas leads to an energy shift and broadening of the spectrum, which are the same for all positron energies. Thus, it is incorrect to use energy calibration for a single section with gamma peaks corresponding to sources and extrapolation of resolution assuming square root dependence for positron energy reconstruction. Further, we will return to discussion of this problem because the energy resolution of positron registration is extremely important for description of the oscillation process.

\section{COMPUTER MODEL OF REACTOR ANTINEUTRINO DETECTOR}

In order to estimate the efficiency of an antineutrino detector, a computer model of the detector was created, and using it, Monte Carlo calculations were carried out. Size of the detector and properties of the IBD process were used as the model parameters. In MC calculations, in the annihilation of stopped positron, two gamma quanta are emitted 

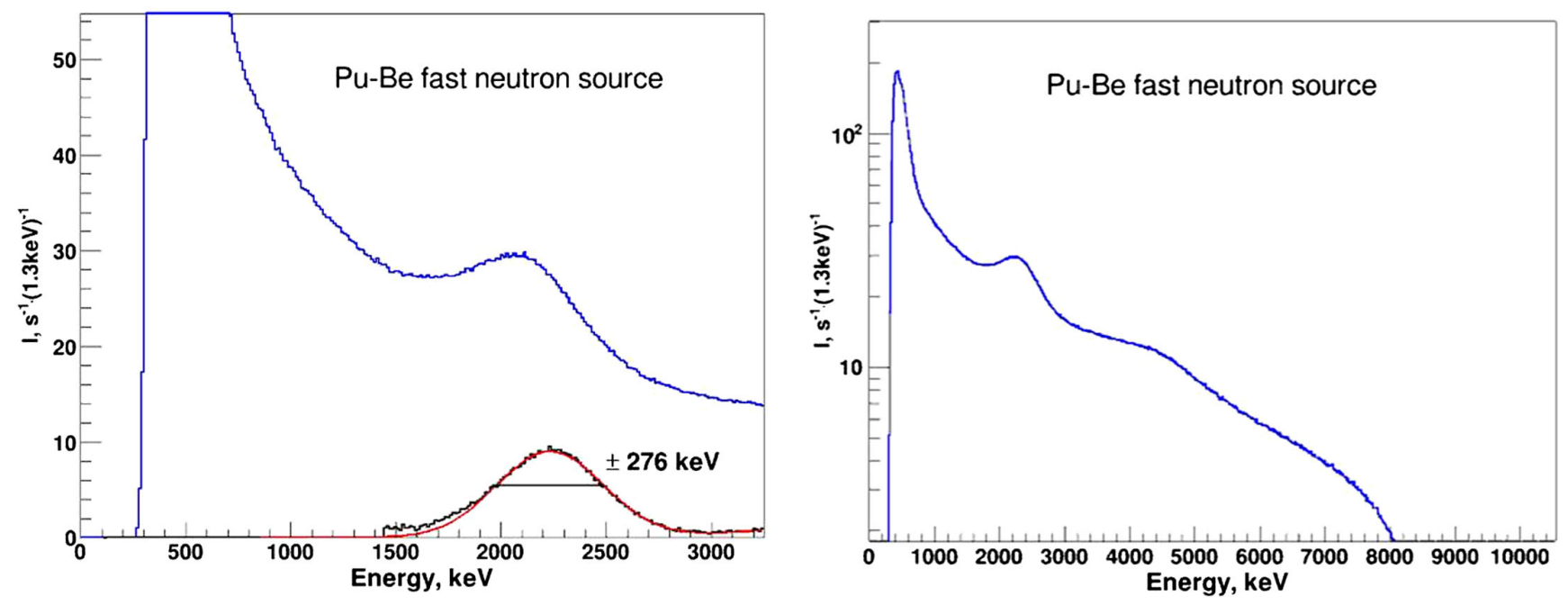

FIG. 21. The results of detector calibration. Left: line 2.2 Mev of np-d $\gamma$ process; Right: $8 \mathrm{MeV}$ from $\mathrm{Gd}(\mathrm{n}, \gamma)$.

with energies $511 \mathrm{keV}$ propagating in the opposite directions. The neutrons from the IBD process are absorbed by $\mathrm{Gd}$ with an emission of a gamma cascade of total energy of $8 \mathrm{MeV}$. The detector registers two successive signals from the positron and neutron. The detector scheme used in the model is shown in Fig. 24.

The detector vessel is divided into $5 \times 10$ sections $0.225 \times 0.225 \times 0.75 \mathrm{~m}^{3}$ with rigidly fixed partitions between them. The scintillator material is mineral oil $\left(\mathrm{CH}_{2}\right)$ doped with $\mathrm{Gd}$ of a concentration $1 \mathrm{~g} / \mathrm{l}$. Scintillator light yield is $10^{4}$ photons per $1 \mathrm{MeV}$. The thickness of the walls was neglected. Hamamatsu R5912 are used in the model. A layer of air separates the PMT from scintillator. The antineutrino spectrum is calculated from the positron spectrum, because to the first order approximation, it can be represented as a linear function: $E_{\bar{\nu}}=E_{e^{+}}+1.8 \mathrm{MeV}$.

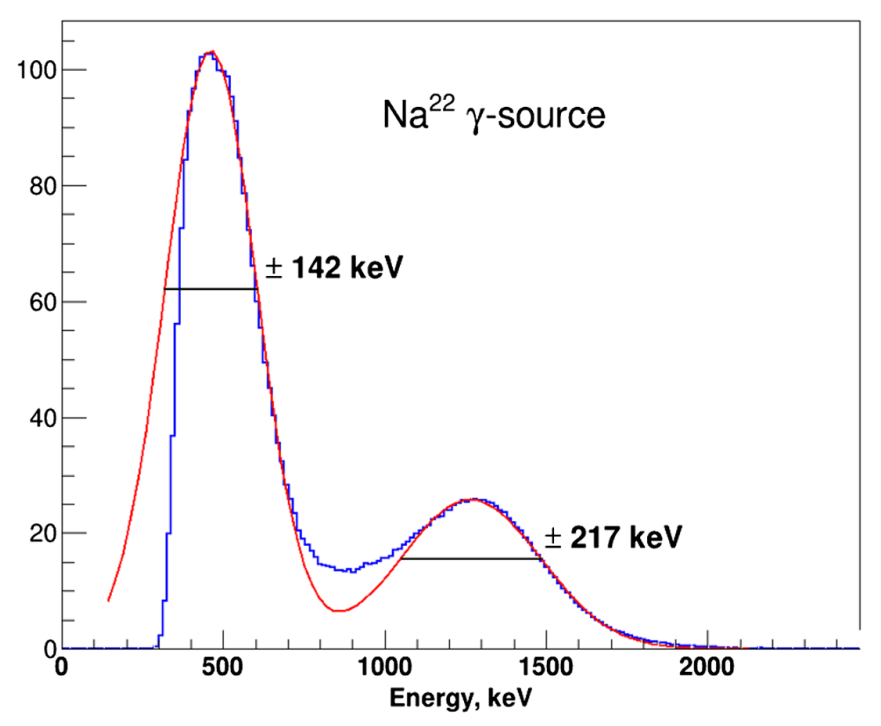

FIG. 22. Calibration with ${ }^{22} \mathrm{Na}$ source.
To simulate the antineutrino spectrum, we used the antineutrino spectrum of ${ }^{235} U$ [30]. In the model, the antineutrino flux has a direction parallel to the detector axis. This approximation is valid for detector distances in the range 6-12 $\mathrm{m}$ from the reactor core. Positron directions have isotropic distribution. The space distribution of neutron captures by $\mathrm{Gd}$ and energy yields of positron and gammas in scintillator were calculated with the MCNP program [33]. The gamma spectrum of neutron capture by $\mathrm{Gd}$ was generated using the spectrum of process ${ }^{157} \mathrm{Gd}(\mathrm{n}, \gamma)$. Exponential track length of a photon into the scintillator is $4 \mathrm{~m}$. The probability of photon reflection from the wall is 0.95 .

A distribution of PMT counts (number of registered photons) from positrons of various energies and two gamma quanta with energies $511 \mathrm{keV}$ is shown in Fig. 25. The top image shows the distribution of signals for one section, taking into account the signals from the registration of two gammas with energies $511 \mathrm{keV}$. It is

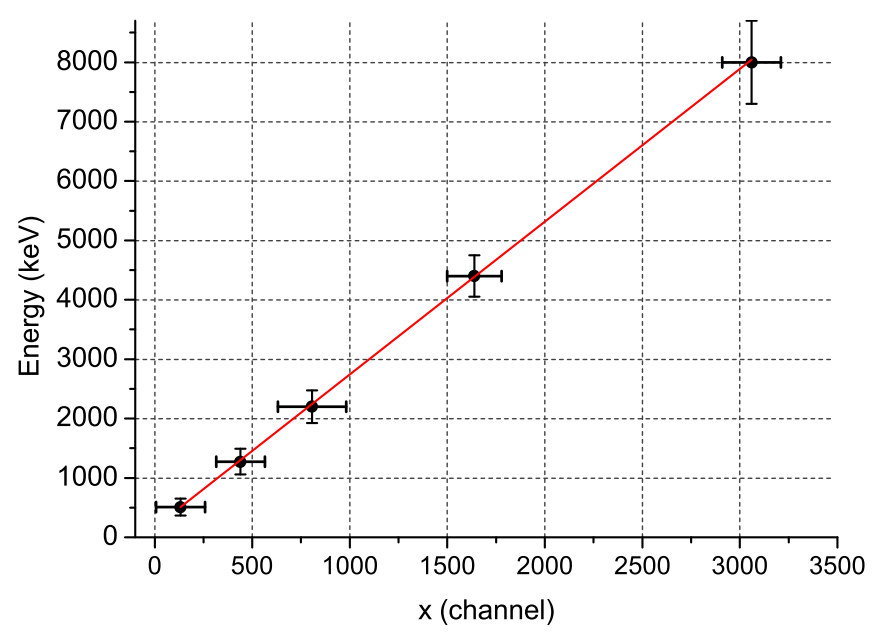

FIG. 23. Linearity of energy calibration. 

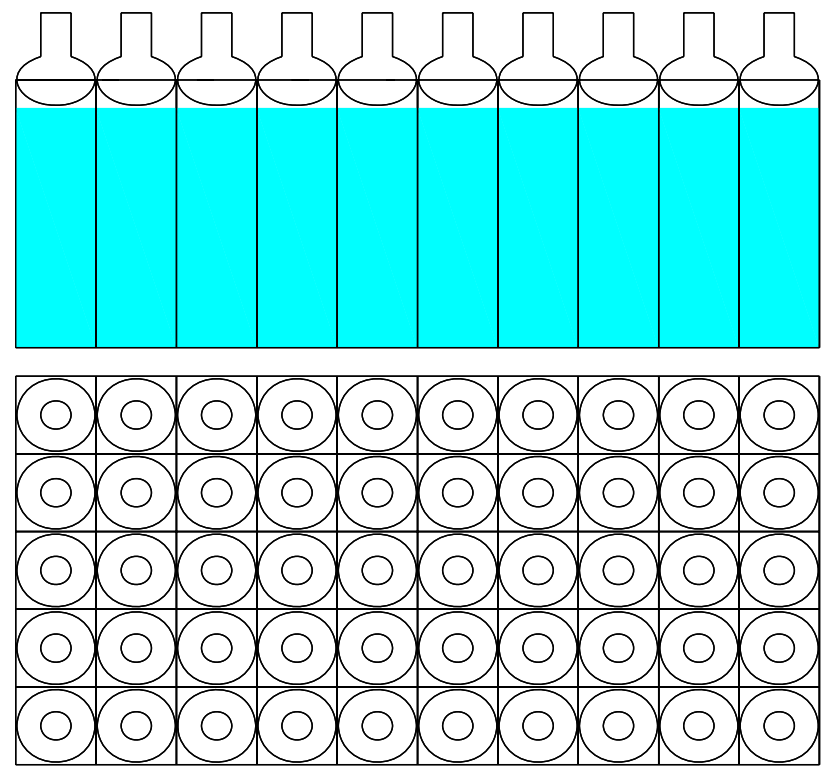

FIG. 24. Scheme of the detector of reactor antineutrino.

mostly determined by the incomplete absorption of gamma quanta within one section. It explains two peaks in distribution at low energies. The middle image illustrates the distribution of signals from a positron only without two gamma quanta from the annihilation process. The distributions from the top image are also present at the middle image for comparison. The bottom image demonstrates the signals, taking into account the annihilation gammas and registration in adjacent sections and distributions from the top image for comparison. This distribution is asymmetrical, and hence, it is difficult to estimate corresponding energy resolution. However, one can take the effective $2 \sigma$ distribution width, which covers $68 \%$ of the total area under the curve. The calculations (Table II) reveal that the effective width obtained from the results presented at bottom image almost does not depend on energy of positrons and can be estimated as $2 \sigma=500 \mathrm{keV}$.

Examples of registration of gamma quanta with energies 2.3 MeV, 4.4 MeV, and 6.0 MeV are shown in Fig. 26 for the case of registration by only one section (green curve) and the case of registration taking into account signals from nearest sections (red curve). The energy resolution in the case of registration in several sections is 2 times better $2 \sigma=250 \mathrm{keV}$ for positron energy 4.4 MeV. The presence of partitions between adjacent sections should decrease the energy resolution. We will return to the question of the energy resolution of the detector in Sec. XIV.

Distributions of signals of positron and neutron events are shown in Fig. 27. The threshold of the positron event is 1.5 MeV. The efficiency of positron registration obtained with taking into consideration the positron spectrum and threshold is $\epsilon_{e^{+}}=0.810(5)$.

The lower limit of energy in the registration of a neutron signal is planned to be set at level $3.2 \mathrm{MeV}$, which will help
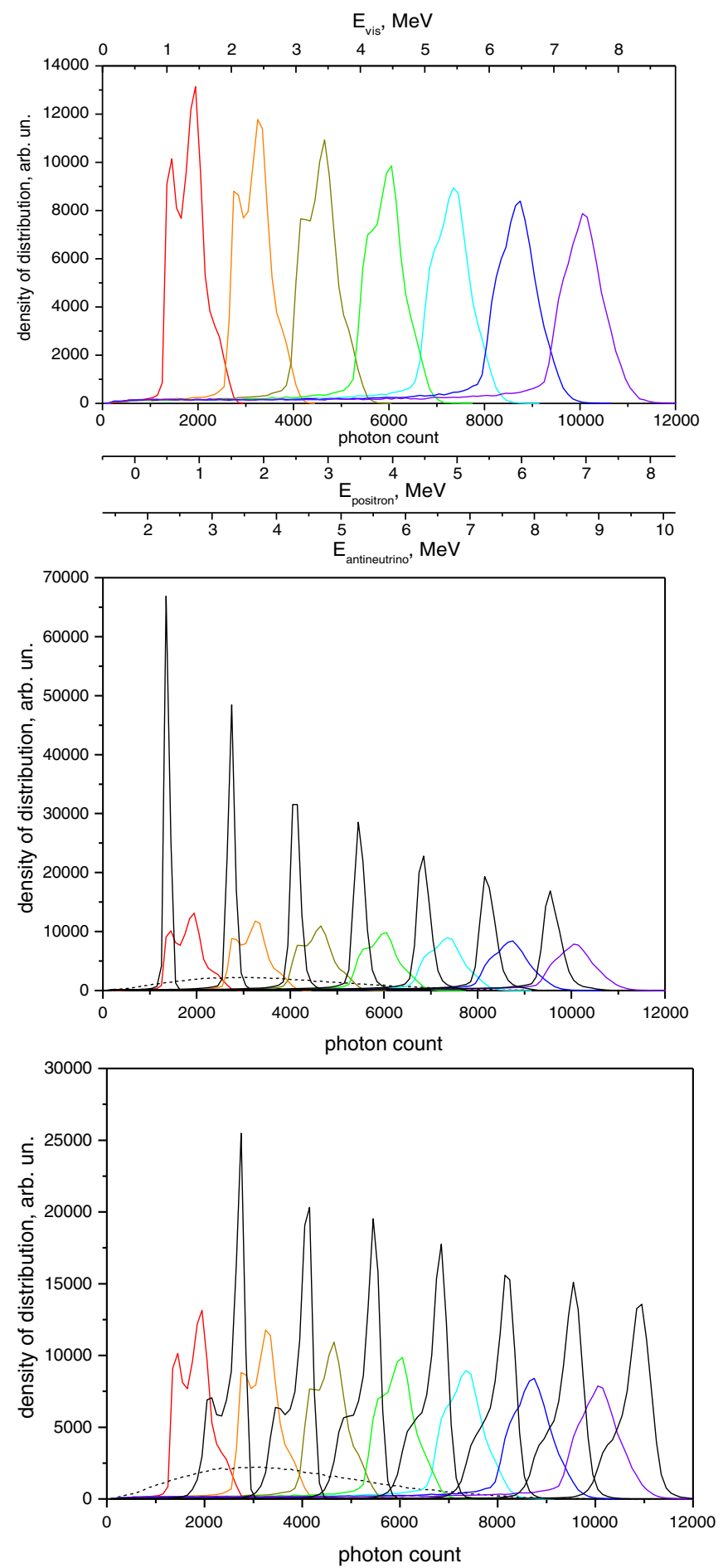

FIG. 25. Distribution of counts of PMT in one section induced by positrons with energies from 1 to $7 \mathrm{MeV}$ with annihilation process (2 gamma-quanta with energies $511 \mathrm{keV}$ ).

us to suppress the accidental coincidence background caused by natural radioactivity [26]. With such a limit, the registration efficiency of the neutron signal from ${ }^{157} \mathrm{Gd}(\mathrm{n}, \gamma)$ is $\epsilon_{n}=0.713(5)$ if we consider counts of PMTs of all sections. If we consider counts of PMT in one section, where the process ${ }^{157} \mathrm{Gd}(\mathrm{n}, \gamma)$ takes place 
TABLE II. Calculated $\sigma$ for the Epos.

\begin{tabular}{lc}
\hline \hline $\mathrm{E}_{\mathrm{pos}}, \mathrm{MeV}$ & $\sigma, \mathrm{keV}$ \\
\hline 2 & 215 \\
4 & 233 \\
6 & 251 \\
\hline \hline
\end{tabular}

$\epsilon_{n}=0.194(5)$. Taking into account the fact that $\sim 20 \%$ of neutrons are captured by hydrogen with an energy yield of $2.2 \mathrm{MeV}$, the efficiency is $\epsilon_{n}=0.570(5)$. The registration efficiency of IBD obtained in the simulation is $\epsilon=0.462(5)$. If we consider only the PMT in the section where the process occurred, then $\epsilon=0.128(5)$. Efficiency of the detector as a

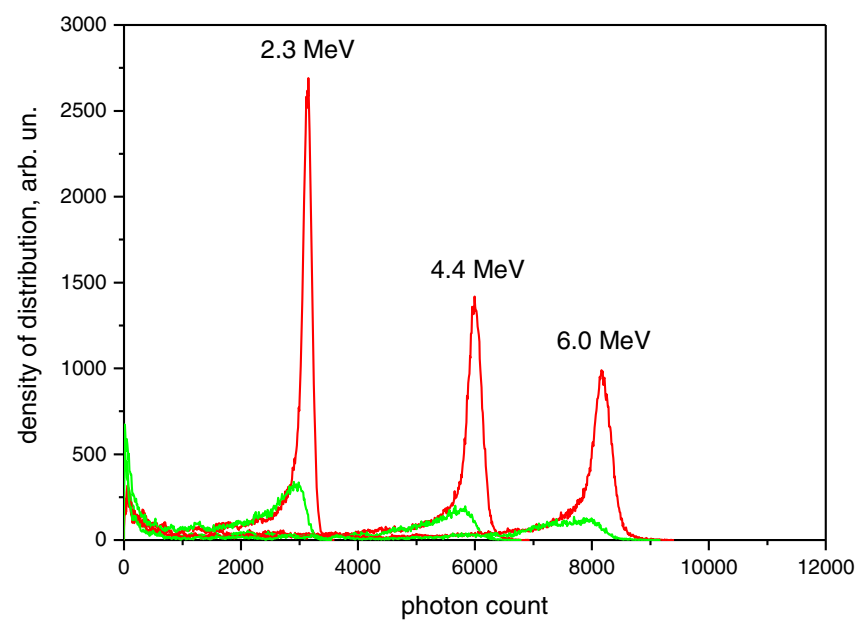

FIG. 26. Distribution of counts of PMT in one section induced by gamma quanta with energies $2.3 \mathrm{MeV}, 4.4 \mathrm{MeV}$, and $6.0 \mathrm{MeV}$.

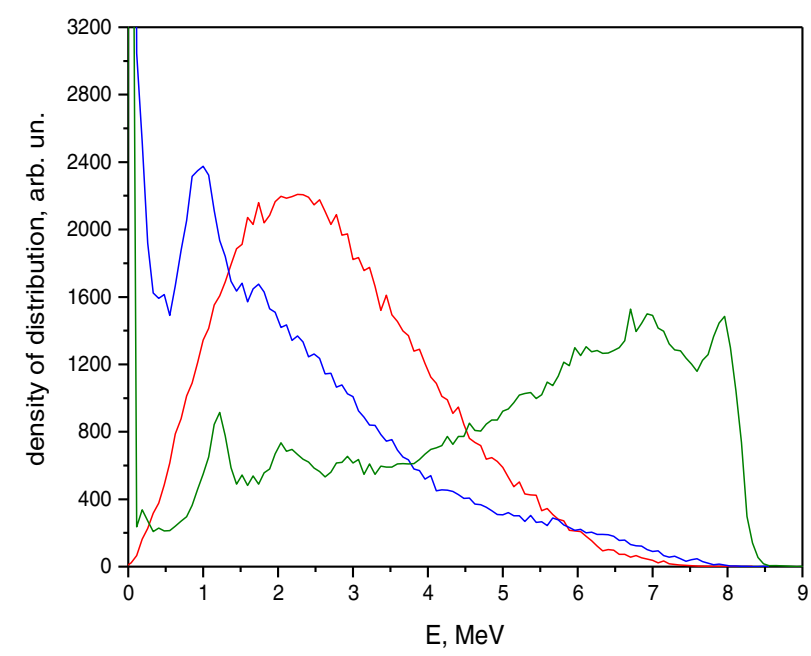

FIG. 27. Signals of positon (positron and 2 gamma-quanta) event-red curve, signals of neutron event-blue curve (only PMT of section where the IBD process took place is used), green curve-signals of PMTs from all sections.

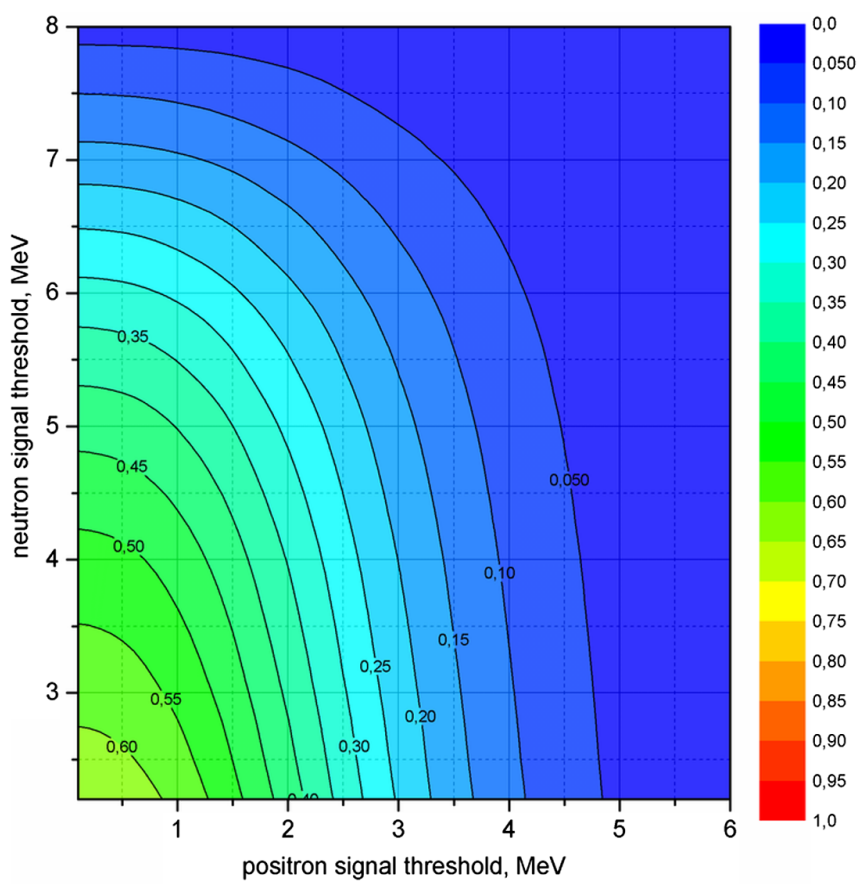

FIG. 28. Efficiency of the detector as function of limits of positron and neutron signals with counts of all PMTs.

function of the limits of positron and neutron signals with counts of all PMTs is shown in Fig. 28.

In the experiment, the limits of positron and neutron signals are set to be $1.5 \mathrm{MeV}$ and $3.2 \mathrm{MeV}$, correspondingly. For those values, the $\mathrm{MC}$ simulation gives a detector efficiency $\sim 46 \%$. If we consider counts from PMTs of all the sections, also consider the influence of materials in the scintillator volume and incomplete signal collection from the process ${ }^{157} \mathrm{Gd}(\mathrm{n}, \gamma)$ in different sections then the detector efficiency can be estimated to be $\sim 20 \%$.

The ratio of the expected neutrino count to the register is $25 \%-30 \%$, which is explained by that estimate.

It should be noted that the accurate estimate of the detector efficiency is not the task of this experiment since we use the method of relative measurements through the use of a mobile detector.

\section{MEASUREMENTS-THE SCHEME OF REACTOR OPERATION AND DETECTOR MOVEMENTS. SIGNAL AND BACKGROUND}

The measurements of the background (off) and the measurements with the reactor in operation mode (on) were carried out for each detector position within a single measuring period. The scheme of reactor operation and detector movements is shown in Fig. 29. A reactor cycle is 8-10 days long. Reactor shutdowns are $2-5$ days long and usually alternate $(2-5-2-\ldots)$. The reactor shuts down in summer for a long period for scheduled preventive maintenance. The movement of the detector to the next measuring position takes place in the middle of reactor 


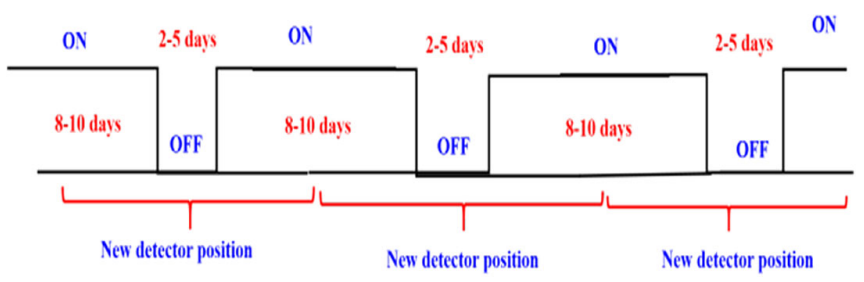

FIG. 29. Scheme of detector operation and detector movements.

operational cycle. Then the measurements are carried out at the same position until the middle of the next cycle. The scheme of detector operation and detector movements is shown on Fig. 29.

The registration rate in the off regime of the reactor is mainly determined by the cosmic background, and the way it changes with distance mainly depends on the structure of the building. At the distances 10-12 m, the blocking of cosmic rays is better because of concrete ceiling as shown in Fig. 2.

The cosmic background is larger than the signal, and it is the main problem of the experiment. It includes the correlated signal, the same the neutrinos produce. Therefore, distribution of cosmic background oscillations with time has to be accurately investigated.

Figure 30 (top) illustrates the pattern of the correlated background obtained during the whole measurement time. That pattern includes corrections for background changes caused by detector movements. The fluctuations of this pattern are mostly statistical. The measurements of the background were carried out by different time intervals, e.g., in 2020, when the reactor was not operating, the measurement intervals were several times longer, and
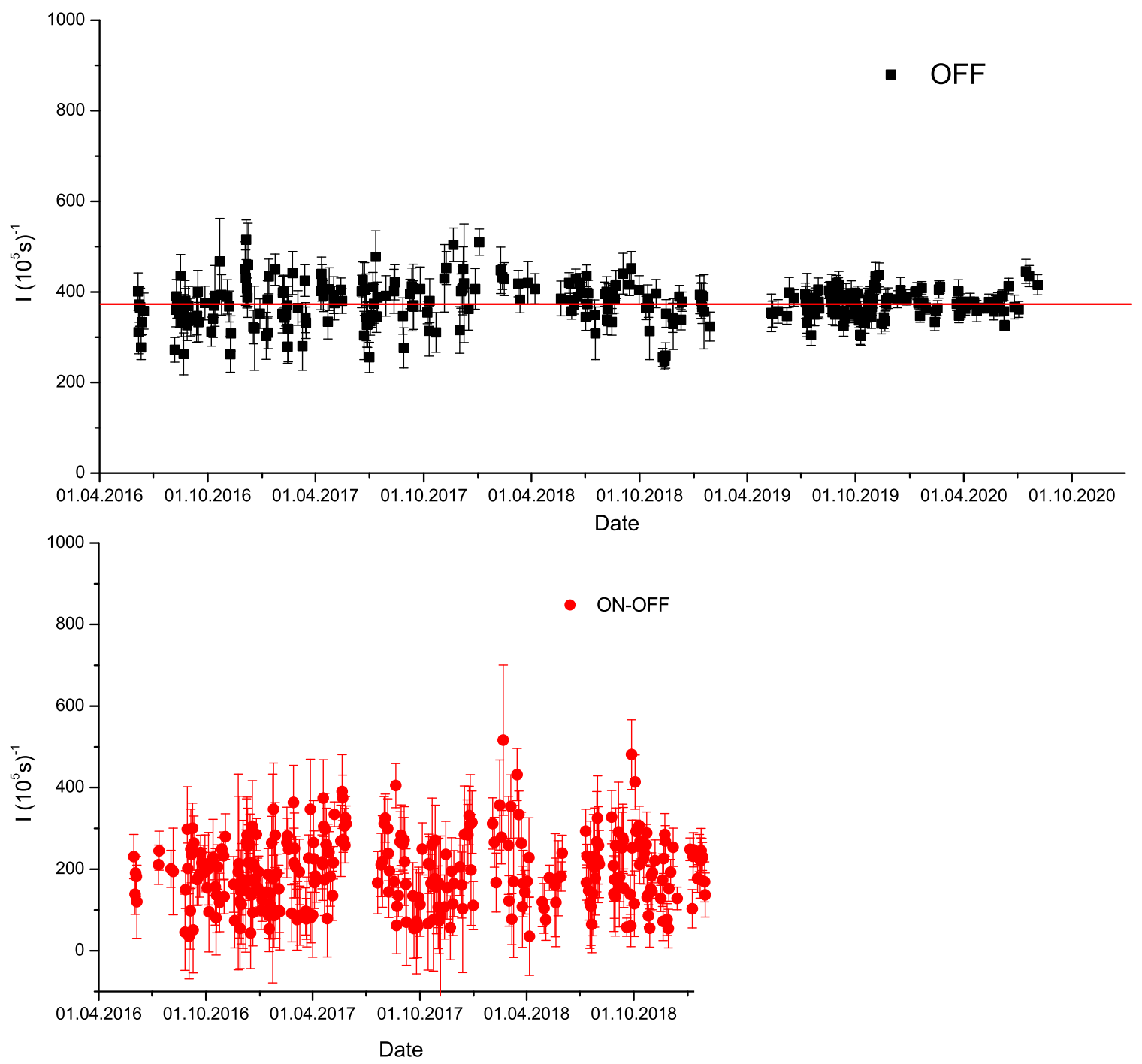

FIG. 30. The correlated signals produced by the cosmic background measured over the whole time (top). The correlated on-off signals over the whole time (bottom). First cycle of measurements is from the beginning to the May 10, 2018. Second cycle is from May 19, 2018 to reactor stopping for the long period for reconstruction. Third cycle is measurements of the background during reactor reconstruction. 
OFF fluctuation distribution

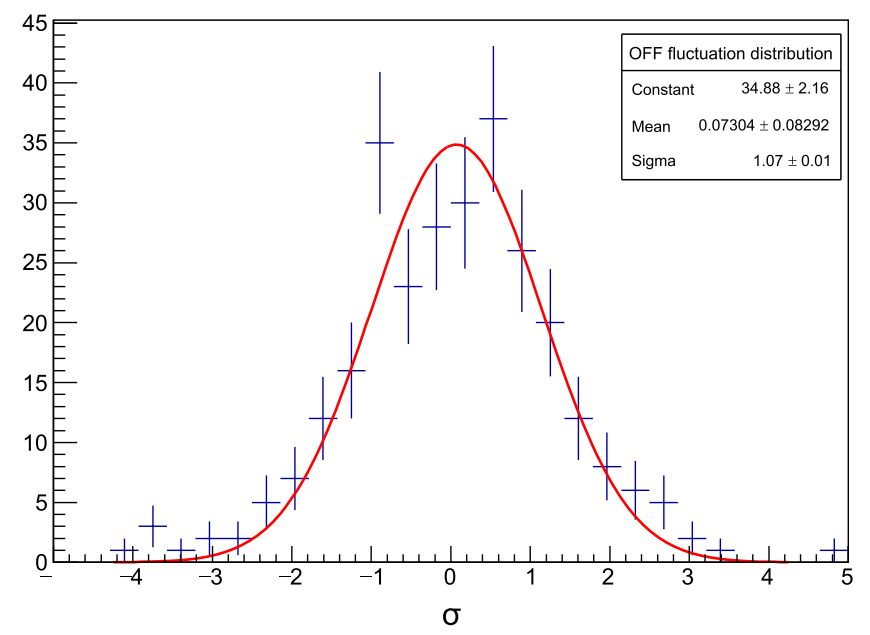

ON-OFF fluctuation distribution

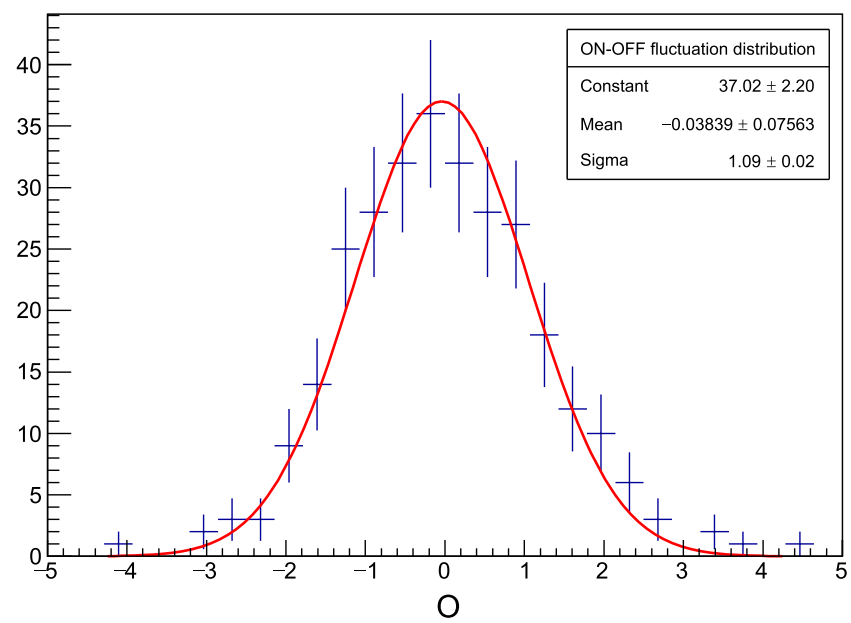

FIG. 31. The distribution of deviations from average value of correlated events rates background (off) and differences (on-off) normalized on their statistical uncertainties.

hence, the fluctuations are significantly lower. To perform a more detailed analysis, one has to construct the distribution of the fluctuations normalized for each point at the corresponding statistical error. This distribution is shown in Fig. 31 (left). The standard deviation of this distribution is $1.07 \pm 0.01$; i.e., the additional fluctuations of cosmic background are only $7 \%$.

The similar analysis of the stability for the on-off signal is shown in Fig. 30 (bottom). The correction for the dependence of the on-off signal on the distance is also applied. Distribution of the fluctuation of it is shown in Fig. 31 (right). As before, the fluctuations are normalized to the corresponding statistical uncertainty of the measurement. By the way, it should be noted that reactor power fluctuations in different cycles are about $2 \%$ and also are averaged out in the long-term measurements. The standard deviation of this distribution is $1.09 \pm 0.02$; i.e., the additional fluctuations of cosmic background reactor power are only $9 \%$.

The broadening in the statistical distribution of the neutrino signal due to fluctuations in the cosmic background and reactor power variation is $9 \%$ of the standard statistical deviation. As a result, we conclude that the measurements can be carried out with almost statistical accuracy despite the rather high cosmic background. In principle, one can add the correction to fluctuations of the cosmic background in each measurement using the fluctuations of atmospheric pressure. However, that is excessive since the correction is small, and the fluctuations of the cosmic background averages out for the order of magnitude because the number of measurements is 87 .

In conclusion, the analysis of the experimental data can be performed using the observed accuracy, which is closed to the statistical one.

\section{SELECTION OF CORRELATED EVENTS}

In order to form the reactor antineutrino signal, one has to select correlated events. That selection technique, including time intervals and topological criteria for IBD events, are presented below. Every signal in $100 \mu$ s after an active shielding event is banned. Prompt (positron) and corresponding delayed (gamma from the neutron captured by gadolinium) signals have to occur in a $300 \mu$ s time window. Events with a time gap less than $100 \mu$ s between the prompt and single delayed signal are considered as correlated. Events with a time gap from 100 to $300 \mu \mathrm{s}$ become a basis for the estimation of the accidental coincidence background, which is subtracted for every measurement.

A prompt signal should be registered in a single detector cell or in two adjacent cells. The threshold for a prompt signal is $1500 \mathrm{keV}$, and for the last case energy, it is applied to the sum of two signals. The threshold for each signal is $200 \mathrm{keV}$. The delayed signal from a few gamma quanta of the $\operatorname{Gd}(\mathrm{n}, \gamma)$ reaction should be from two or more cells, which are not three cells far from the prompt signal cell in any direction. That natural condition helps us to decrease theaccidental coincidence background. The experimental distribution of delayed signals after IBD event in section $(4,3)$ is shown in Fig. 32.

\section{BACKGROUND SPECTRUM}

The background is one of the main problems of the experiment, and it should be investigated in detail. The correlated background occurs due to fast neutrons according to the scheme shown in Sec. VIII Fig. 13, where the prompt signal is formed by the recoil proton emitted in the elastic scattering of a neutron on hydrogen, which is one of the main components of the scintillator. But the neutrons 


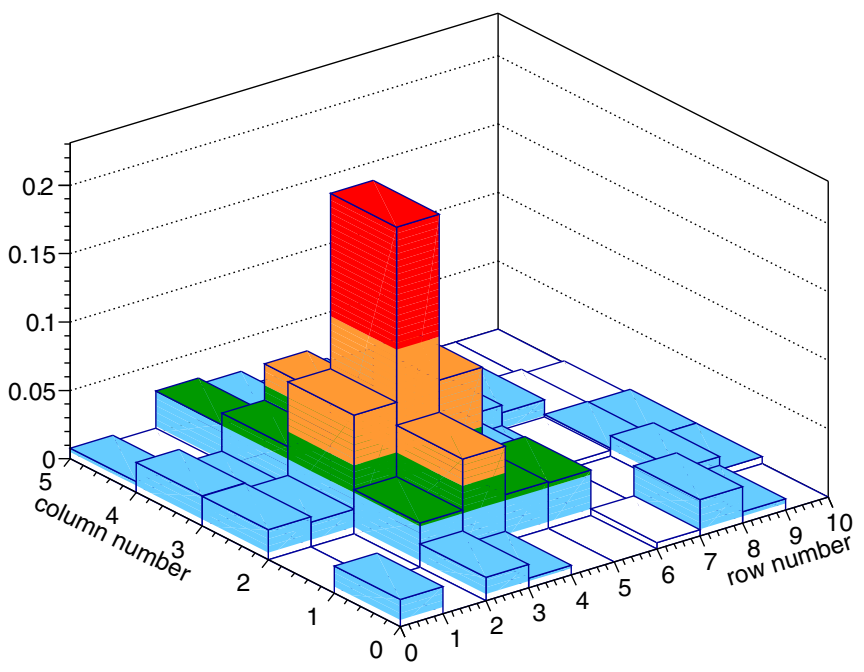

FIG. 32. Experimental distribution of delayed signals after the prompt signal occurred in section $(4,3)$.

can also interact with carbon nuclei, which are also present in the scintillator. Moreover, neutrons can be inelastic scattered on the oxygen nuclei in the acrylic glass, which is the main component of the mirror walls of the sections, and on the aluminum nuclei in the detector shell or section separations, which are made of the aluminum alloy (see Fig. 19).

The spectrum of the background averaged over all the distances is shown in Fig. 33. The area 3-6 MeV has a bump and irregular behavior of the background dependences on the energy (Fig. 33). That behavior can be explained by the structure of energy levels of the carbon, oxygen, and aluminum nuclei. Those irregularities of the spectrum superpose with the smooth spectrum function of the recoil protons emitted in elastic scattering of fast neutrons on the hydrogen and correlated background produced by unstable isotopes ${ }^{9} \mathrm{Li}$ and ${ }^{8} \mathrm{He}$, which are the result of the interaction of cosmic rays (muons) with carbon nuclei. These isotopes decay in the electron channel with the following decay in which a neutron is emitted.

A fast neutron in the $\left(n, n^{\prime}\right)$ process leaves the nucleus exited and its deexcitation occurs before the neutron can be thermalized and captured. The energies of the two lowest levels of the carbon nucleus are $4.44 \mathrm{MeV}$ and $7.65 \mathrm{MeV}$. The excitation of the level $7.65 \mathrm{MeV}$ is possible with fast neutrons with energies above $8.25 \mathrm{MeV}$. The level 7.66 is mainly decayed into three alpha particles through the intermediate nucleus of ${ }^{8} \mathrm{Be}$ [34]. The total energy of the alphas is about $400 \mathrm{keV}$. The first exited state $4.44 \mathrm{MeV}$ is a result of the inelastic scattering of the fast neutron and decay with emission of a gamma before the neutron can be thermalized and captured. Therefore, we can observe the correlated event. In the case of the oxygen nucleus, the most intensive line has energy 6.13 MeV. The same scheme in the case of the aluminum nucleus leads to gamma quanta with energies $2.2 \mathrm{MeV}, 3.68 \mathrm{MeV}, 4.4 \mathrm{MeV}$, and 5.15 MeV.

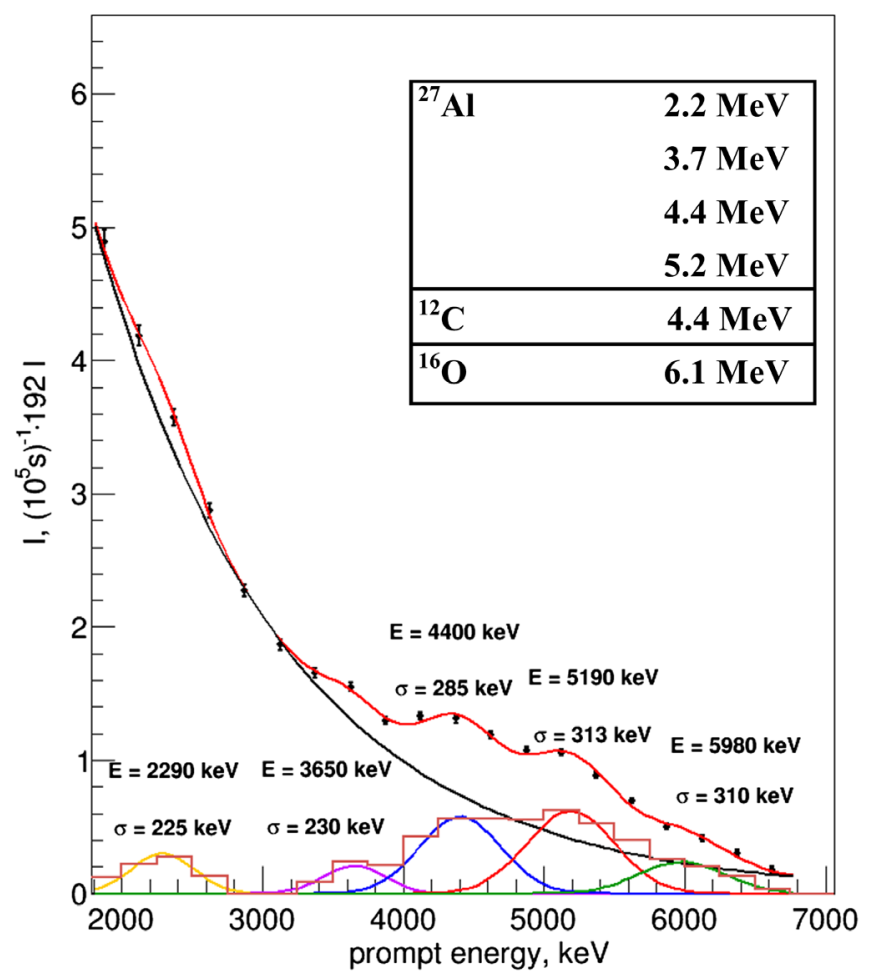

FIG. 33. Background spectrum averaged over all distances. Up-range $1500 \div 7000 \mathrm{keV}$, down-range $2500 \div 7000 \mathrm{keV}$.

At varying degrees of confidence, it can be said that we observe the gammas with energies: $2.2 \mathrm{MeV}, 3.68 \mathrm{MeV}$, 4.44 MeV, 5.15 MeV, and 6.13 MeV.

The presence of that structure in the energy spectrum indicates that the energy calibration of the detector was the same in all the measurements. Moreover, since we observe the separate lines in the spectrum, though the resolution is rather low, we still can estimate the energy resolution of the detector and suggest the decomposition of the background spectrum into the set of gamma lines (see Fig. 33). In the range from $2.0 \mathrm{MeV}$ to $7.0 \mathrm{MeV}$, the energy resolution changes from $225 \mathrm{keV}$ to $310 \mathrm{keV}$. The estimated value corresponds to the resolution of the whole detector, which is better than the resolution of the single section, because of taking into account the signals in adjacent section.

In order to verify the estimation of the detector energy resolution that we used in the decomposition of the measured background energy spectrum, we performed a MC calculation of the energy resolution of the whole detector. The examples of the simulation results for gammas with energies $2.3 \mathrm{MeV}$, $4.4 \mathrm{MeV}$, and $6 \mathrm{MeV}$ are shown in Fig. 26. The calculated resolution is $2 \sigma=$ $250 \mathrm{keV}$ at an energy of $4.4 \mathrm{MeV}$. But in the experiment, the resolution at this energy is $2 \sigma=570 \mathrm{keV}$, i.e., 2 times worse. The partitions between the sections, difference in calibration of the sections, and instability in the three years long measurements can decrease the energy resolution. 
XV. ANTINEUTRINO SPECTRUM (ON-OFF) AND SPECTRUM OF ACCIDENTAL COINCIDENT

In order to obtain an antineutrino spectrum as the difference of on-off spectra, we subtract contributions of both processes with an uncertainty equal to fluctuations of the cosmic background, which, as was discussed before, are only $7 \%$ of the statistical accuracy of a single measurement, and they are averaged out for an order of magnitude during the long-term measurements. The on-off difference is 223 events per day in distance range $6-9 \mathrm{~m}$. The signal/ background ratio is 0.52 .

Figure 34 illustrates two spectra of correlated signals with the reactor on and off (background), and also the onoff spectrum. As was discussed above, their difference describes an antineutrino spectrum of the reactor. The accuracy of that statement is based on the fact that the changes of fast neutron flux on the surface of the containment building of the reactor, which can contribute in the onoff difference do not exceed $(1.1 \pm 0.45) \%$ as discussed in
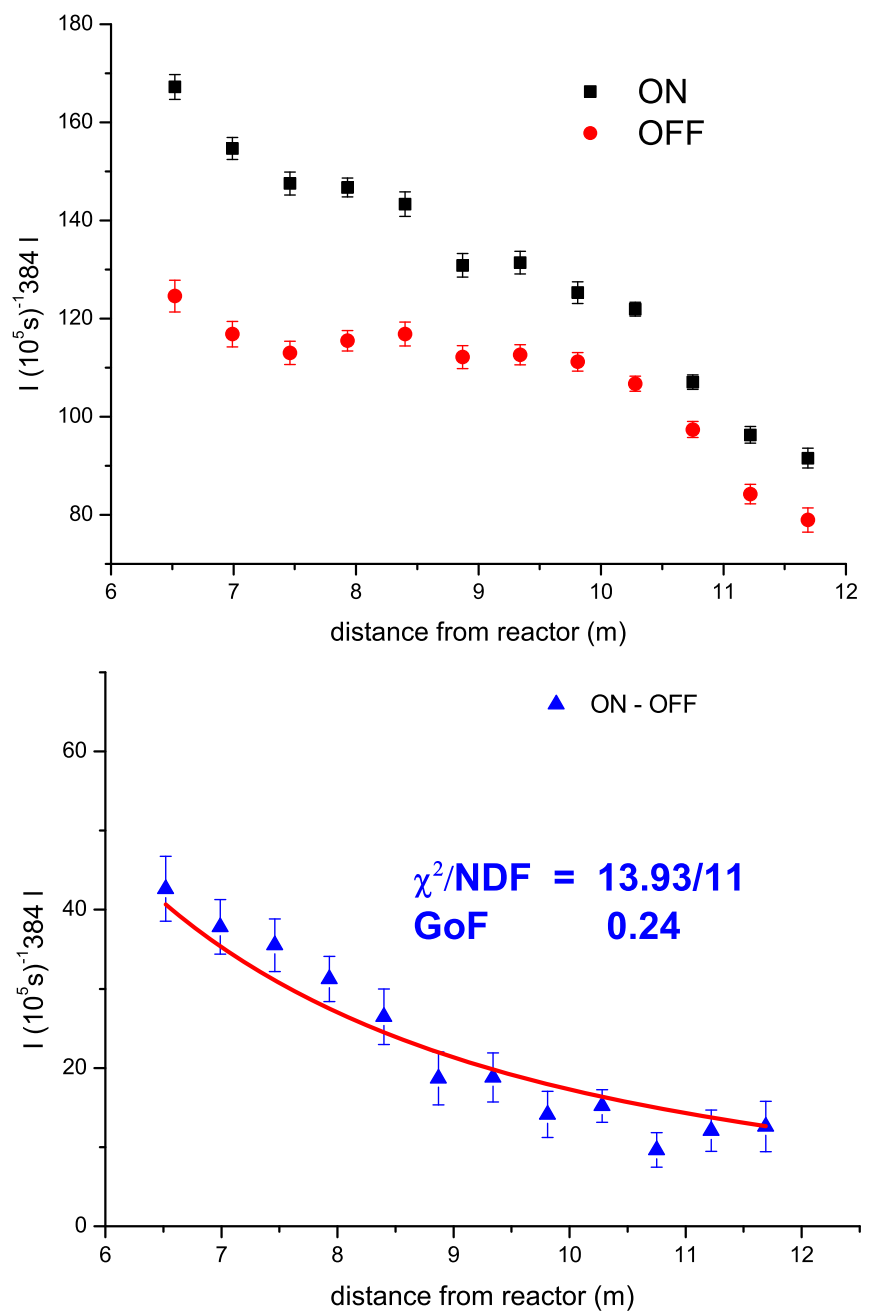

FIG. 34. Up: event rate with operating (on) and stopped (off) reactor. Down: difference between event rates (on-off) as functions of distance.
Sec. V. Moreover, this is not a problem for these measurements, especially since this background cannot have the oscillation behavior. The event rate with operating (on) and stopped (off) reactor, and also the difference of that rates (on-off) as functions of distance are shown in Fig. 34.

Figure 35 (top) illustrates the on and off spectra averaged over all distances. Figure 35 (bottom) illustrates on-off signals averaged over all distances - blue histogram and the difference of averaged spectra for on and off signals-red histogram. Notice, that in the analysis of the antineutrino signal, one has to use an on-off signal for each distance obtained in the nearest measurements. That way, one obtains the best possible compensation of the fluctuations of the cosmic background with time.

Besides the correlated background, there is also a problem of an accidental coincidence background. The energy spectrum of the accidental coincidence background is shown in Fig. 36 (up) for three distances. The influence of the reactor operation mode on the accidental coincidence background is also shown in Fig. 36 (down). The background significantly increases if we decrease the limit of delayed signals under $3 \mathrm{MeV}$ level, and even some
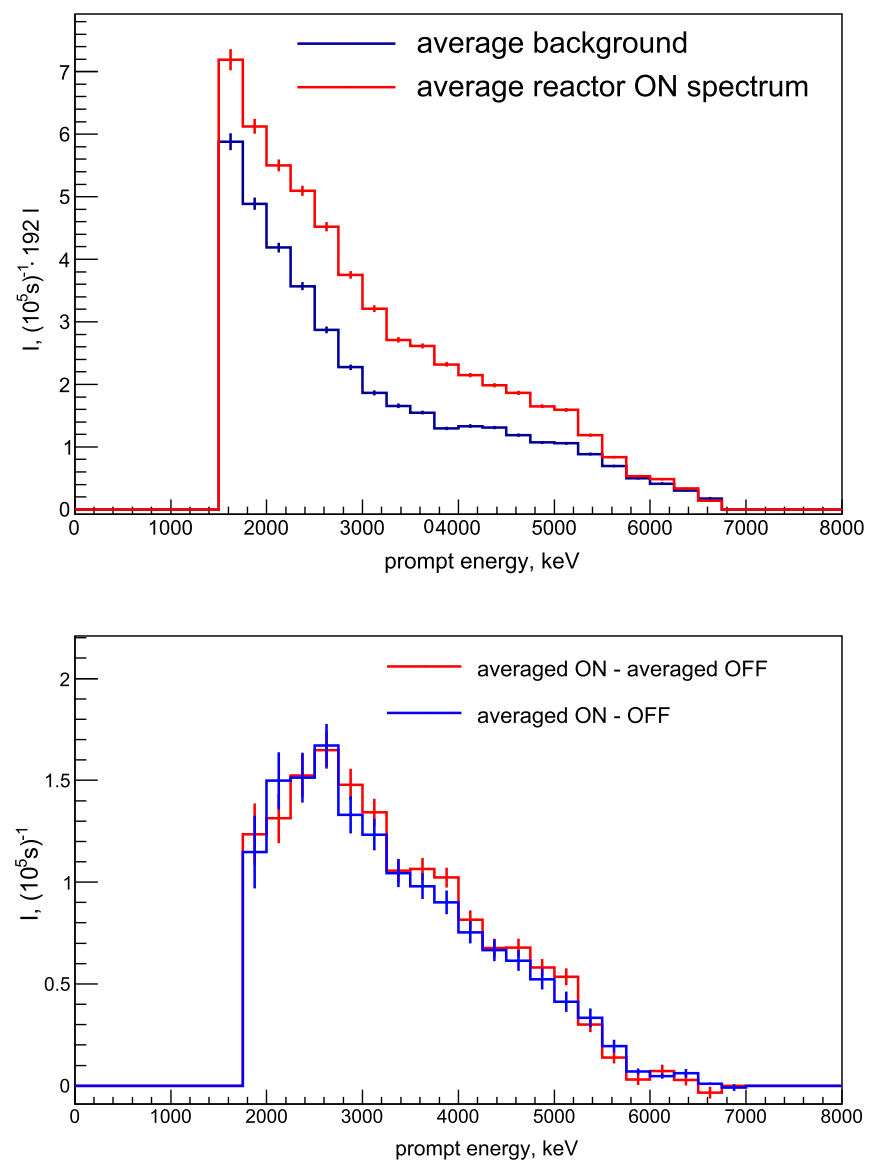

FIG. 35. Top: the on and off spectra averaged over all distances. Bottom: on-off signals averaged over all distances-blue histogram and the difference of averaged spectra for on and off signals-red histogram. 


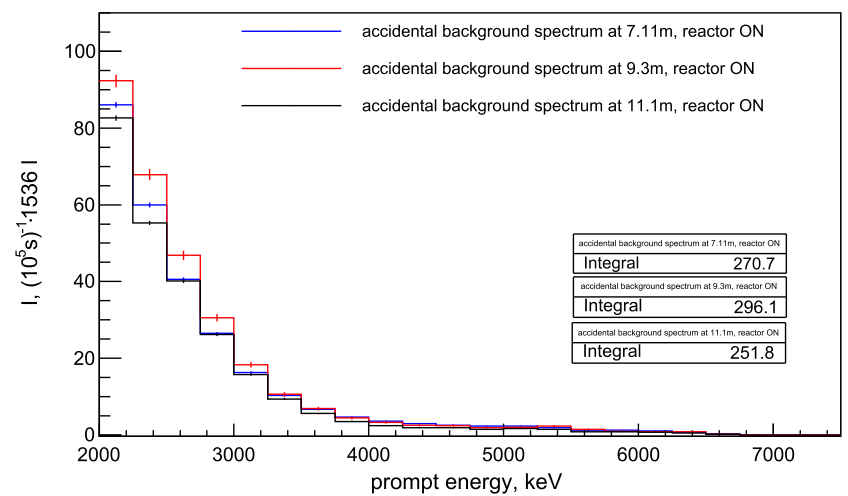

Accidental background at $7.11 \mathrm{~m}$ from reactor.

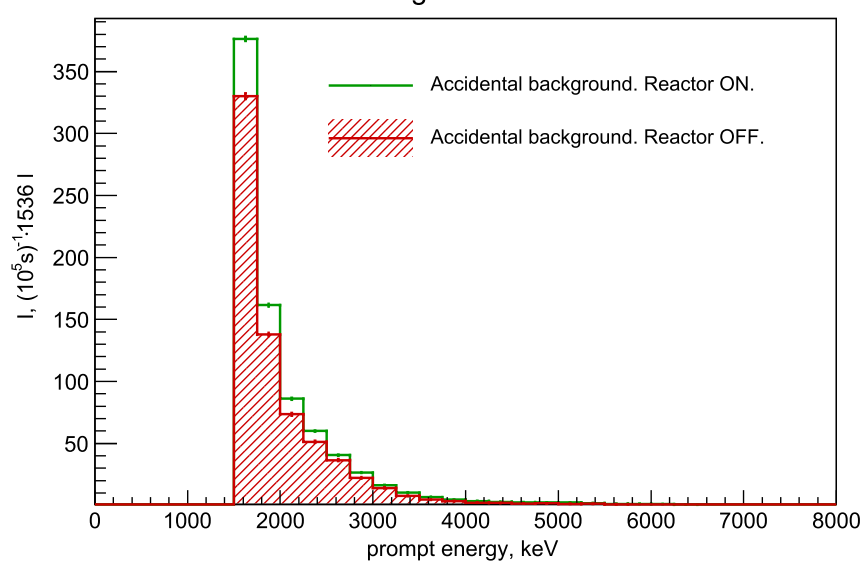

FIG. 36. The spectra of accidental coincidence background at $7.11 \mathrm{~m}, 9.3 \mathrm{~m}$, and $11.1 \mathrm{~m}$ (up). The on and off spectra at the point closest to the reactor-7.11 $\mathrm{m}$ (down).

dependence of reactor mode can be observed. As was discussed before, this problem can be solved by setting a lower limit of the delayed signal to be $3.2 \mathrm{MeV}$, because neutron capture by Gd yields signal with sufficiently high energy up to $8 \mathrm{MeV}$, while the natural radioactivity background is almost zero above $3 \mathrm{MeV}$. The limit of the prompt signal we set at level $1.5 \mathrm{MeV}$ significantly decreases the amount of cutoff of neutrino events.

Thus, the conclusion of this analysis is the selection of the optimal lower thresholds for prompt and delayed signals: $1.5 \mathrm{MeV}$ and $3.2 \mathrm{MeV}$, respectively.

\section{COMPARISON OF EXPERIMENTAL ANTINEUTRINO SPECTRUM WITH CALCULATED REACTOR SPECTRUM}

In order to compare the experimental spectrum of antineutrinos with the calculated spectrum of reactor antineutrinos, one has to consider the results of $\mathrm{MC}$ calculations of the efficiency of registration of IBD process in the detector. The spectrum obtained in the experiment corrected by the efficiency factor should be compared with expected spectrum of the prompt signals calculated in the MC simulation. An example of such a comparison is shown in Fig. 37, where we present the experimental spectrum of prompt signals, averaged over all distances for better statistical accuracy, and the MC spectrum of prompt signals, obtained using the spectrum of ${ }^{235} \mathrm{U}$ and taking into consideration the threshold of experimental signals.

As was already pointed out, the correct way of analysis for neutrino oscillations implies the usage of the averaged on-off signal spectrum for each distance; moreover, it had better be obtained in the nearest measurements, in order to better compensate for temporal fluctuations of the cosmic background. However, a comparison can also be made using the difference between the on and off spectra averaged over all distances. This allows us to include in the data processing long-term background measurements obtained in 2020. Both spectra then can be compared with the calculated one. Results of such a comparison are shown in Fig. 37. Compensation of fluctuations of the cosmic background is better for the measurements with a short time between the reactor on and reactor off period.

The comparison of the experimental and calculated spectra demonstrates a slight deviation from the theoretical picture within current statistical uncertainty. The reasons for the possible discrepancy may be due to different circumstances. First, the energy calibration of the calculated neutrino spectrum is not fixed, and the energy dependence of the detection efficiency is not completely accurate. Second, one can consider the presence in the spectrum of the often discussed bump in the $5 \mathrm{MeV}$ region. Both reasons cannot be ruled out. To avoid this problem, a spectrally independent method for analyzing experimental data is needed. Further, we propose such an analysis method.

\section{SPECTRAL INDEPENDENT METHOD FOR ANALYSIS OF EXPERIMEHTAL DATA}

Here, we suggest a spectral independent method of data analysis based on relative measurements, in which the spectrum is canceled out. Therefore, it is a model independent analysis of data. It is based on Eq. (3), where in the left-hand side, the numerator is the rate of antineutrino events in a certain energy area at a certain distance with the correction to the geometric factor $\mathrm{L}^{2}$ and the denominator is the rate of antineutrino events with the same energy, but it is averaged over all distances,

$$
\begin{aligned}
R_{i k}^{\exp } & =\left(N_{i k} \pm \Delta N_{i k}\right) L_{k}^{2} / K^{-1} \sum_{k}^{K}\left(N_{i k} \pm \Delta N_{i k}\right) L_{k}^{2} \\
& =\frac{\left\langle S(E)\left(1-\sin ^{2} 2 \theta_{14} \sin ^{2}\left(\frac{1.27 \Delta m_{14}^{2} L_{k}}{E}\right)\right)\right\rangle_{i}}{K^{-1} \sum_{k}^{K}\left\langle S(E)\left(1-\sin ^{2} 2 \theta_{14} \sin ^{2}\left(\frac{1.27 \Delta m_{14}^{2} L_{k}}{E}\right)\right)\right\rangle_{i}}
\end{aligned}
$$

$S(E)$-initial ${ }^{235} \mathrm{U}$ spectrum, $\langle s\rangle$-is the integration with the function of energy resolution with $\sigma=250 \mathrm{keV}$ and integration over energy bin intervals. The right part of the 

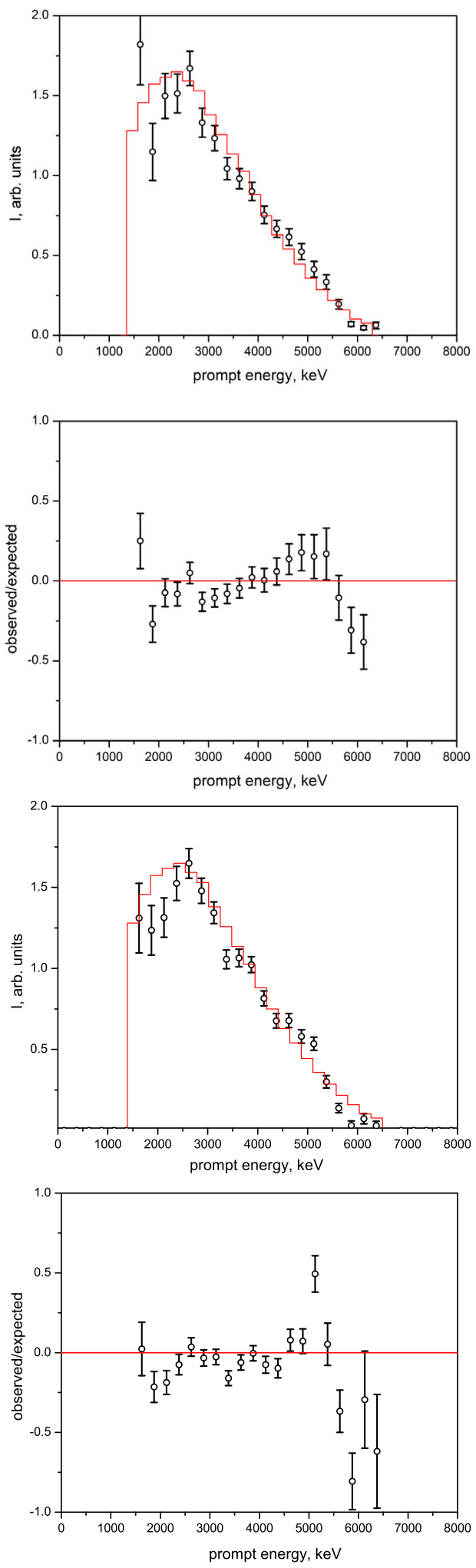

FIG. 37. Comparison of the calculated MC spectrum of antineutrino for ${ }^{235} \mathrm{U}$ with the experimental on-off spectrum. Below their difference normalized to the calculated spectrum is shown. The first two figures represent the on-off signal spectrum averaged over all distances; the second two figures represent the difference between the on and off spectra averaged over all distances, where long-term background measurements are included. equation is the same ratio written in analytical form taking into account the oscillation hypothesis. There was an estimation that showed the right-hand side of the equation practically does not depend on the function $S(E)$ [28].

The denominator of the ratio is the rate of antineutrino events with the same energy, but it is averaged over all distances; hence, the oscillation effect is considerably averaged out in the denominator if oscillations are frequent enough in the considered distances range. In this case, it is

$$
R_{i k}^{\mathrm{th}} \approx \frac{1-\sin ^{2} 2 \theta_{14} \sin ^{2}\left(1.27 \Delta m_{14}^{2} L_{k} / E_{i}\right)}{1-\frac{1}{2} \sin ^{2} 2 \theta_{14}} \underset{\theta_{14}=0}{\rightarrow} 1,
$$

In the no oscillation case, this expression becomes equal to unity. The $R_{i k}^{\exp }$ ratio in approximation (3) with an accuracy to the coefficient $1 /\left(1-1 / 2 \sin ^{2} 2 \theta_{14}\right)$ corresponds to the Eq. (1), which is describing the process of oscillations.

As will be shown later, the oscillation parameters found in the experiment satisfy approximation (3), although Eq. (2) is used in all the procedures of the oscillation analysis.

At the end of this section, it should be emphasized that the proposed data processing method greatly simplifies the requirements for the energy calibration of the detector and determining its efficiency, as follows from the $R_{i k}^{\exp }$ ratio. Moreover, this method allows us to directly demonstrate the process of oscillations.

The moveable detector method is also a method of relative measurements, so their joint use significantly increases the reliability in the revelation of the oscillation process.

Figure 38 demonstrates the scheme that is applied to construct the dependence on the parameter L/E using the matrix of the experimental data and the process of coherent summation.

\section{MONTE CARLO SIMULATION OF RESULTS EXPECTED WITH EMPLOYING OF SPECTRAL INDEPENDENT METHOD OF DATA ANALYSIS}

In this section, we present results of the MC simulation in which we incorporated geometric configuration of the antineutrino source and detector, including the sectioning. For example, in this simulation, we have used parameters $\Delta \mathrm{m}_{14}^{2}$ and $\sin ^{2} 2 \theta_{14}$, close to the values that will be derived from the analysis of experimental data later. The goal of this simulation is to see what the process of oscillations on the plane $(E, L)$ looks like and how to extract the process of oscillations as a dependence on the L/E ratio according to the Eq. (1).

The source of an antineutrino with geometrical dimensions of the reactor core $42 \times 42 \times 35 \mathrm{~cm}^{3}$ was simulated as well as a detector of the antineutrino taking into account its geometrical dimensions (50 sections of 


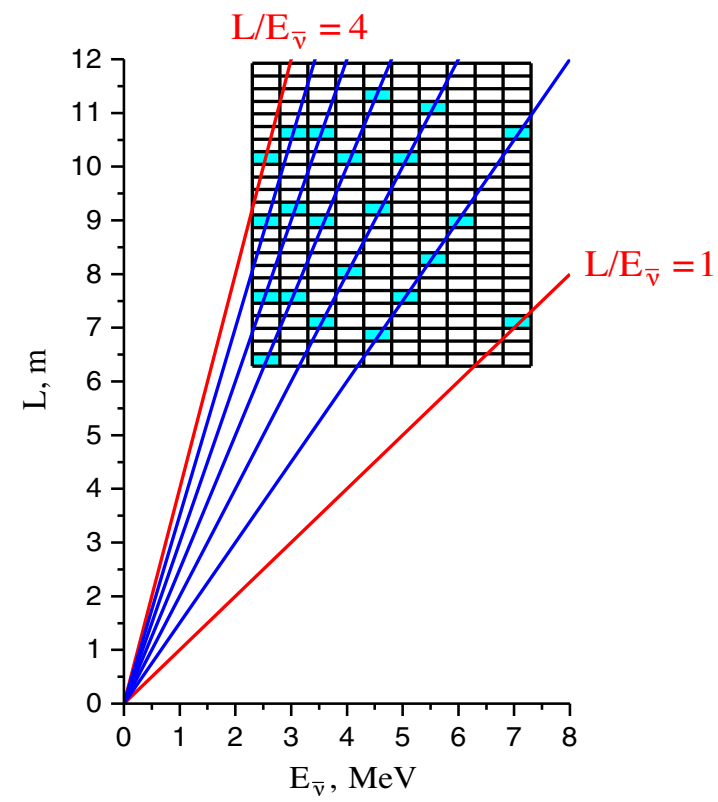

FIG. 38. Illustration of the method of coherent summation of measurement results to obtain the dependence of $R_{i k}^{\text {sim }}$ on the ratio $\mathrm{L} / \mathrm{E}$.

$\left.22.5 \times 22.5 \times 75 \mathrm{~cm}^{3}\right)$. The antineutrino spectrum of $\mathrm{U}^{235}$ [though it does not matter since energy spectrum in Eq. (2) is canceled out] factored by the function of oscillations $1-\sin ^{2} 2 \theta_{14} \sin ^{2}\left(1.27 \Delta m_{14}^{2} L_{k} / E_{i}\right)$ was used.

The expected oscillation effect for the different energy resolution of detector is shown in Figs. 39 and 40 on the top as the plane $(E, L)$ and at the bottom as a function of the $\mathrm{L} / \mathrm{E}$ ratio obtained by adding data with the same L/E ratio. This procedure was illustrated in Fig. 38. Figures 39 and 40 (on the top) illustrate the simulated matrix of $R_{i k}^{\text {sim }}$ ratio $\left(N_{i k} L_{k}^{2} / K^{-1} \sum N_{i k} L_{k}^{2}\right)$, where the oscillation process according to the formula (1) is considered. In the simulation, the statistical accuracy of ratio $\Delta N_{i k} / N_{i k}$ is equal to $1 \%$, which is significantly better than the experimental value.

The most important parameter in this simulation was the energy resolution of the detector, which is determined by the energy step in the matrix $250 \mathrm{keV}$ and $500 \mathrm{keV}$, correspondingly. It can be seen that the degradation of the energy resolution of the detector suppresses the observed effect of oscillations, but the number of observed oscillation periods decreases, and the amplitude of the first observed oscillations is still the same.

The MC simulation results can be summarized in several conclusions. First of all, it is possible to get an idea of that as the effect of the oscillations for the $R_{i k}^{\exp }$ relation on the $(E, L)$ plane looks. Secondly, it becomes clear how to directly observe the effect of oscillations.

In this section, we would like to clarify some aspects of experimental data processing and the MC simulations. The problem of energy resolution requires special attention. The construction of the matrix of measurements on
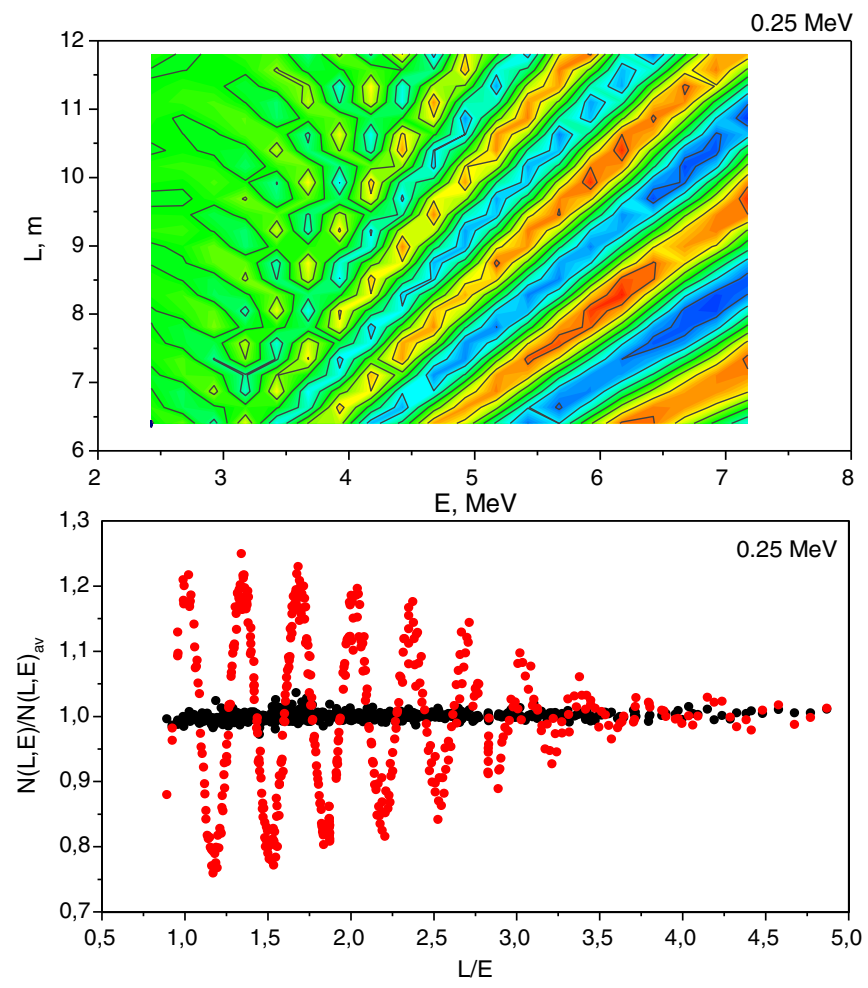

FIG. 39. The simulated matrix of $R_{i k}^{\text {sim }}$ ratio $\left(N_{i k} L_{k}^{2} / K^{-1} \sum N_{i k} L_{k}^{2}\right)$ for energy resolutions of detector $\pm 125 \mathrm{keV}$ (top); dependence of $R_{i k}^{\mathrm{sim}}$ from L/E ratio (bottom).

the $(E, L)$ plane with an energy interval $\Delta E$ includes the energy resolution. The only difference between results presented in the Figs. 39 and 40 is the energy bin intervals, which were $\Delta E=250 \mathrm{KeV}$ and $\Delta E=500 \mathrm{KeV}$. The original simulation of the dataset was performed with assumption of the ideal detector energy resolution. In order to correctly choose the energy interval, we performed the simulation described below.

We simulated the experiment with an energy resolution $\sigma= \pm 250 \mathrm{keV}$. The data processing is performed with energy intervals $125 \mathrm{keV}, 250 \mathrm{keV}$, and $500 \mathrm{keV}$. For comparison, we also performed calculations with ideal energy resolution and different energy intervals.

The comparison of left and right images in Fig. 41 demonstrates the influence of detector energy resolution on the oscillation curve. The right images show that if one set the energy interval smaller or equal to energy resolution $\sigma=250 \mathrm{keV}$ then that choice has almost no effect on the oscillation curve. If one sets the interval to be $\Delta E=$ $500 \mathrm{KeV}$ then it substitutes the detector energy resolution $\sigma=250 \mathrm{keV}$ and such an interval can be used for fast analysis of the data. In the final analysis, one has to use the actual detector energy resolution $\sigma=250 \mathrm{keV}$. The simultaneous usage of the energy interval $\Delta E=500 \mathrm{KeV}$ and energy resolution $\sigma=250 \mathrm{keV}$ is incorrect, because it includes into the analysis the resolution of the detector twice as it was done in the work [35]. 

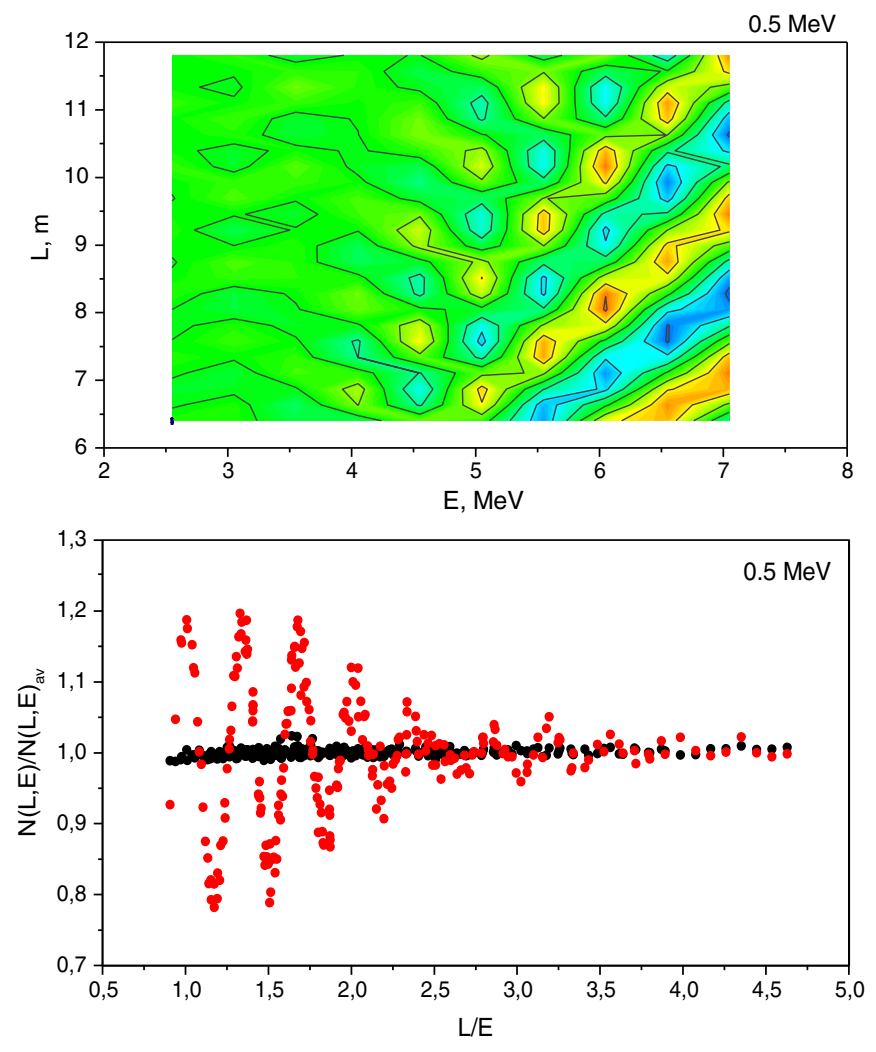

FIG. 40. The simulated matrix of $R_{i k}^{\text {sim }}$-ratio $\left(N_{i k} L_{k}^{2} / K^{-1} \sum N_{i k} L_{k}^{2}\right)$ for energy resolutions of detector $\pm 250 \mathrm{keV}$ (top); dependence of $R_{i k}^{\text {sim }}$ from L/E ratio (bottom).

In summary, on the basis that the estimated detector energy resolution for the gamma quanta registration is in the range $220-310 \mathrm{keV}$ (see Fig. 33) and features of the positron registration (see Fig. 25), we argue that the right way to process the data for the oscillation analysis is by usage of the energy resolution of the detector $\sigma=250 \mathrm{keV}$, which does not depend on the energy of a positron.

\section{THE MATRIX OF MEASUREMENTS}

The results of experimental measurements of the antineurino flux dependence on the distance and energy of antineurino can be presented in the form of a matrix, where $N_{i k}$ is difference of on-off rates for the $i$ th interval of energy and for the $k$ th distance from the reactor core. In total, there are 24 positions of antineurino spectral flux measurements from $6.4 \mathrm{~m}$ to $11.9 \mathrm{~m}$. The distance step corresponds to the cell size of $23 \mathrm{~cm}$, which is twice smaller than the reactor core size.

The energy spectrum is divided using various intervals such as $500 \mathrm{keV}, 250 \mathrm{keV}$, and $125 \mathrm{keV}$. The choice of a smaller interval is unreasonable because it would lead to decrease of statistical accuracy of calculated matrix elements. Even with the $500 \mathrm{keV}$ interval, the obtained statistical accuracy is insufficient to construct the oscillation curve. The large statistical uncertainties obscure the variations in the oscillation amplitude. Therefore, we have to sum the close points of the $\mathrm{L} / \mathrm{E}$ dependence, like it was shown schematically in Fig. 38. This procedure is valid if the summation does not suppress the amplitude of the oscillation.

In further analysis, we use the $500 \mathrm{keV}$ interval and sum the groups of eight adjacent points of the L/E dependence. In addition, we use $250 \mathrm{keV}$ intervals and 16 point groups, and finally, $125 \mathrm{keV}$ intervals and 32 point groups. The black points at the curve (Fig. 41) are the result of summation of the points.

This exact case is illustrated in the right image in Fig. 41 (right) The number of red points corresponds to the number of matrix elements. The black points are the result of the summation of close points of the L/E dependence. The number of black points is the same for all three curves. These three ways of data processing use almost the same data, and the results are equal within statistical accuracy. The processing of the same data 3 times is reasonable because it helps to avoid the problem of fluctuations in the random data sample by averaging over the results of the three described analyses. It is important that the statistical accuracy of the averaged result do remain the same. The total results of the described data analysis procedure are listed below.

For comparison with theory, the MC simulation of the experiment should be carried out, and the energy resolution of the detector should be taken into account. As mentioned in the previous section, the processing of data for the analysis of the oscillation process should be carried out using the energy resolution of the detector $\sigma=250 \mathrm{keV}$, independent of the positron energy.

\section{MEASUREMENTS}

Here, we present the results of the analysis of all data collected from June 2016 till October 2020. On July 2019, the reactor was stopped for renovation. However, background has been measured from July 2019 till October 2020. It was of great importance since reactor shutdowns are 2-3 times shorter than the operational periods. Measurements from September 2018 to July 2019 were carried out mainly in near positions to the reactor, where the signal to background ratio is significantly better. Overall measurements with the reactor on were carried out for 720 days, and with the reactor off for 860 days. Reactor on and off operations occurred 87 times.

It is convenient to divide the measurements in three measurement cycles. The first cycle was carried out between June 2016 and May 2018. The results were published in Ref. [28]. It was the first observation of the oscillation effect at the level of 3 standard deviations. The second cycle was carried out from May 2018 to October 2019. During that period, the statistical accuracy was increased by the factor 1.4. The result of processing of the data obtained in both periods confirmed the oscillation 

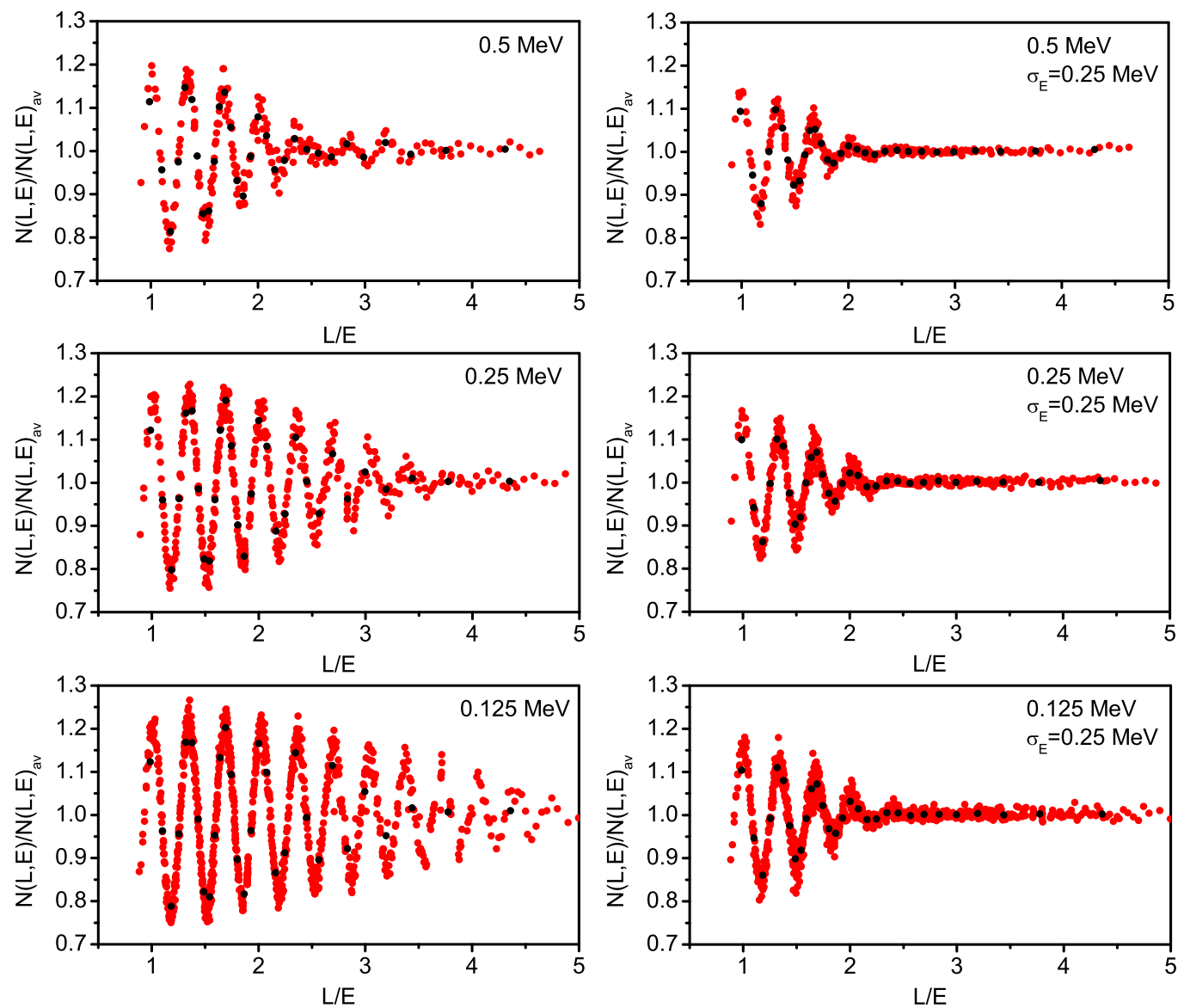

FIG. 41. Left: the simulation of oscillation curve in assumption of the ideal energy resolution and intervals $125 \mathrm{keV}, 250 \mathrm{keV}$, and $500 \mathrm{keV}$. Right: the simulation of oscillation curves with energy resolution of the detector with $\sigma=250 \mathrm{keV}$ and intervals 125,250 , $500 \mathrm{keV}$.

effect with the same confidence level. The results were published in Ref. [36]. In the third cycle, only the measurements of the background were carried out. In this 1.5 year long period, the reactor was stopped for reconstruction. The data obtained in the third measurement cycle are currently included in this article.

Below, we present the results of the described above scheme of the analysis applied to the data obtained in the first measurement cycle, the data obtained in the first and second cycles, and the data obtained in all three measurement cycles.

It is necessary to notice that measurements in the mode when reactor on and reactor off periods are close in time are preferable. In this case, fluctuations of the background are compensated. If we use long and independent measurements of the background, and we compare them to measurements obtained with an operating reactor with an interval of 1-2 years, then we can observe the fluctuations of the background data considerably outside statistical accuracy. As it will be shown further, usage of long-term measurements during the reactor reconstruction is unreasonable if we aim to observe the oscillation effect. It leads only to increase, but not reduce of the uncertainties of measurements. Nevertheless, these measurements were very useful to the detailed study of the background.

\section{THE FIRST PHASE OF DATA ANALYSIS}

Here, we present the data analysis of the reactor antineutrino signal for the first and second measurement cycles combined.

Initial distribution of the count rate $\mathrm{ON}-\mathrm{OFF}=N_{\nu}$ in the entire energy range is shown in Fig. 42. It is the count rate deviation from the mean value for the different series of measurements normalized to its statistical uncertainties. It allows us to combine all measurement results at different distances to find an additional dispersion beside statistical. As can be seen from Fig. 42 (left), it shows a normal distribution determined practically by statistics. It means that additional sources of instabilities besides the cosmic background fluctuations are absent.

We compare it with the distribution obtained for the ratio $R_{i k}^{\exp }$ for the same dataset. It is a difference of $R_{i k}^{\exp }$ from 1 as well as the distribution on-off, normalized by $\sigma$.

In this case, the whole energy range was divided into nine intervals $(500 \mathrm{keV})$, and distances were divided into 

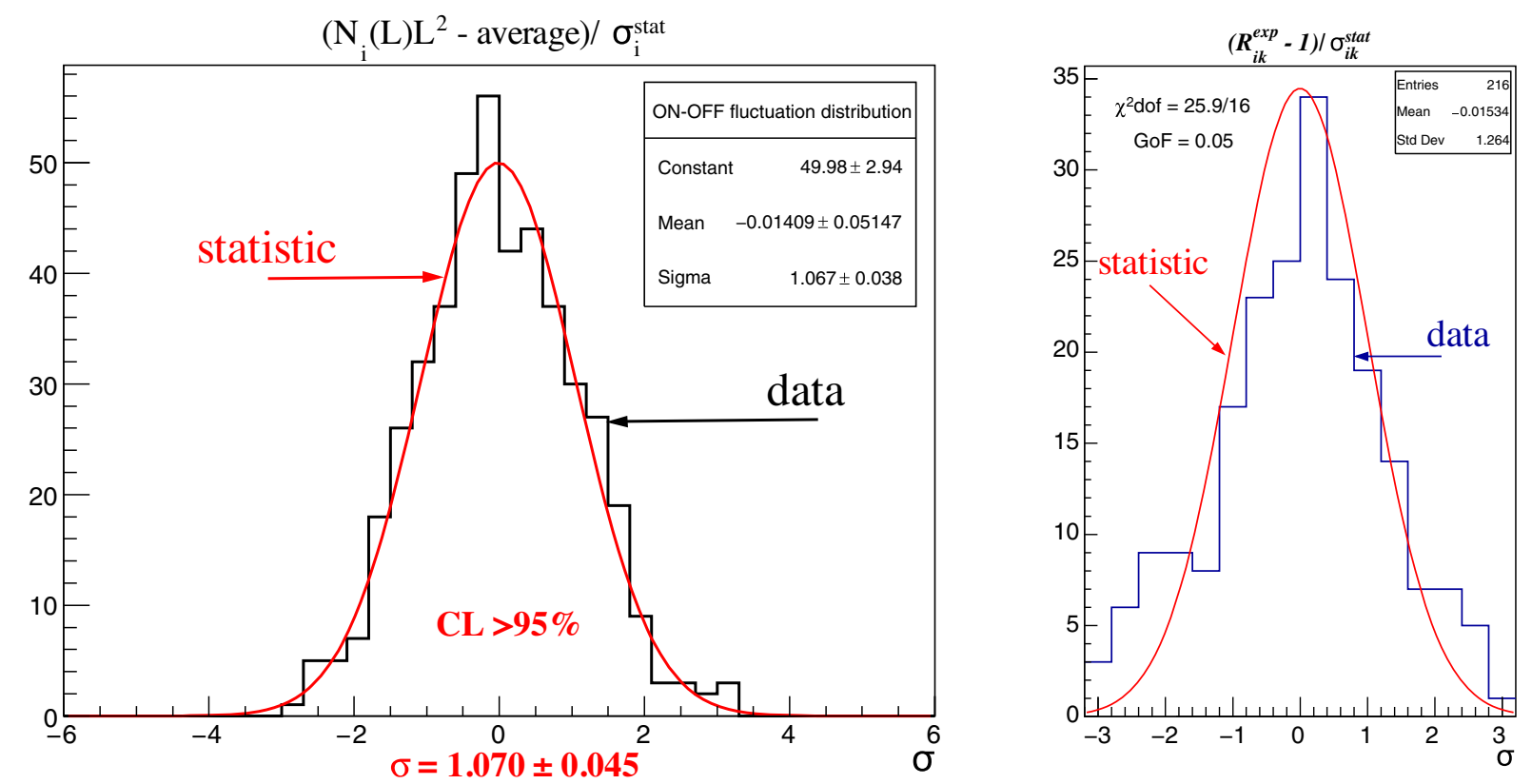

FIG. 42. Left: distribution of the count rate on-off in the entire energy range, normalized by $\sigma$. Right: distribution $R_{i k}^{\exp }$ of all 216 points over the L/E range from 0.9 to 4.7 , normalized by $\sigma$.

24 intervals, so the matrix has 216 elements. Figure 42 (right) shows the distribution of all 216 points over the $\mathrm{L} / \mathrm{E}$ range from 0.9 to 4.7 . One can see that the distribution $R_{i k}^{\exp }$ already differs from normal. The value of the $\chi^{2}$ d.o.f. parameter is $25.9 / 16$, which disfavors this function because the confidence level for this result is only $5 \%$. The additional width of the $R_{i k}^{\exp }$ distribution could appear due to the oscillation effect. It can be considered as first evidence of the oscillation effect, which broadens this distribution.

\section{THE ANALYSIS OF OSCILLATION PROCESS ON THE PLANE $\Delta m_{14}^{2}, \sin ^{2} 2 \theta_{14}$}

The most optimal oscillation parameters can be found by using the expression,

$$
\sum_{i, k}\left(R_{i k}^{\mathrm{exp}}-R_{i k}^{\mathrm{th}}\right)^{2} /\left(\Delta R_{i k}^{\mathrm{exp}}\right)^{2}=\chi^{2}\left(\sin ^{2} 2 \theta_{14}, \Delta \mathrm{m}_{14}^{2}\right) .
$$

The matrix of measurements should be compared with the MC calculated matrix for the detector energy resolution

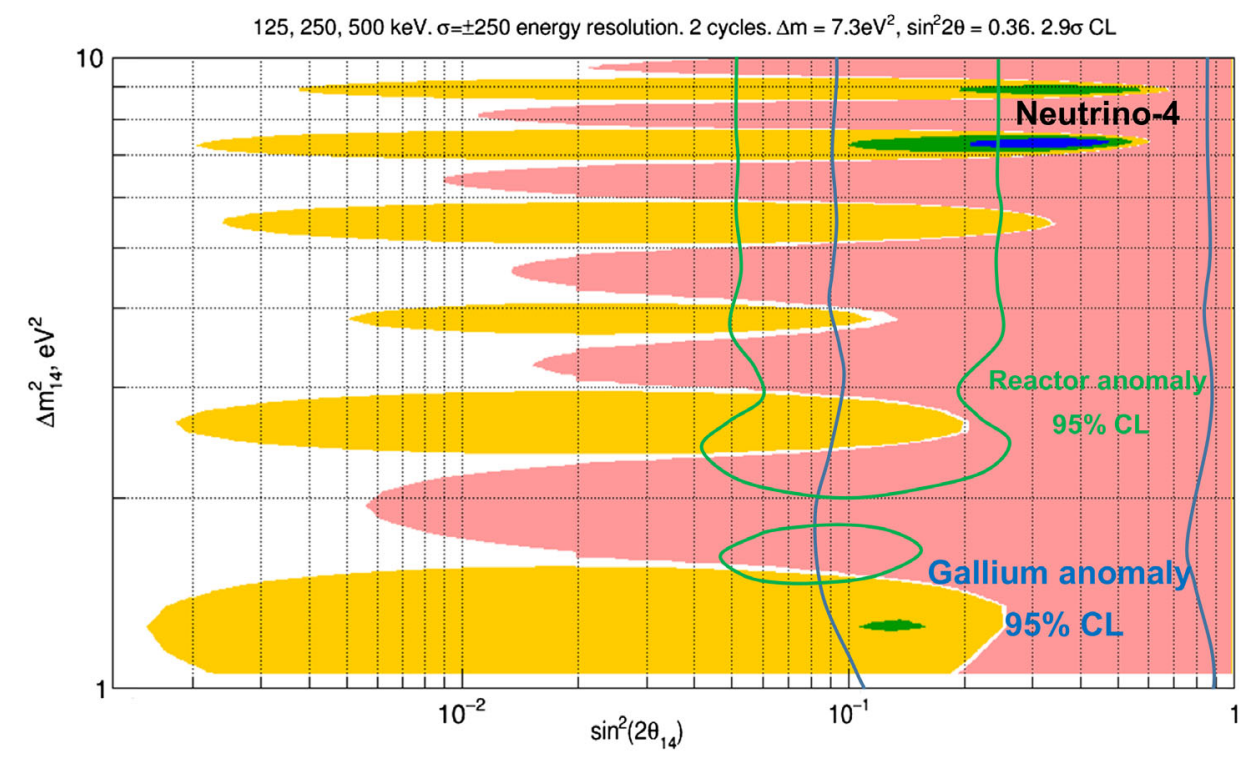

FIG. 43. The results of the analysis of data obtained in first and second measurement cycles on the plane $\Delta m_{14}^{2}, \sin ^{2} 2 \theta_{14}$. 
$\pm 250 \mathrm{keV}$. Below we present the search for oscillation parameters on the plane $\Delta m_{14}^{2}, \sin ^{2} 2 \theta_{14}$ using the $\Delta \chi^{2}$ method and the dependence of the $\mathrm{R}$ ratio on the parameter L/E, i.e., the oscillation curve.
The results of the analysis of data obtained in the first and second measurement cycles on the plane $\Delta m_{14}^{2}$, $\sin ^{2} 2 \theta_{14}$ are presented in Fig. 43. The colored in pink area of oscillation parameters is excluded with a C.L.
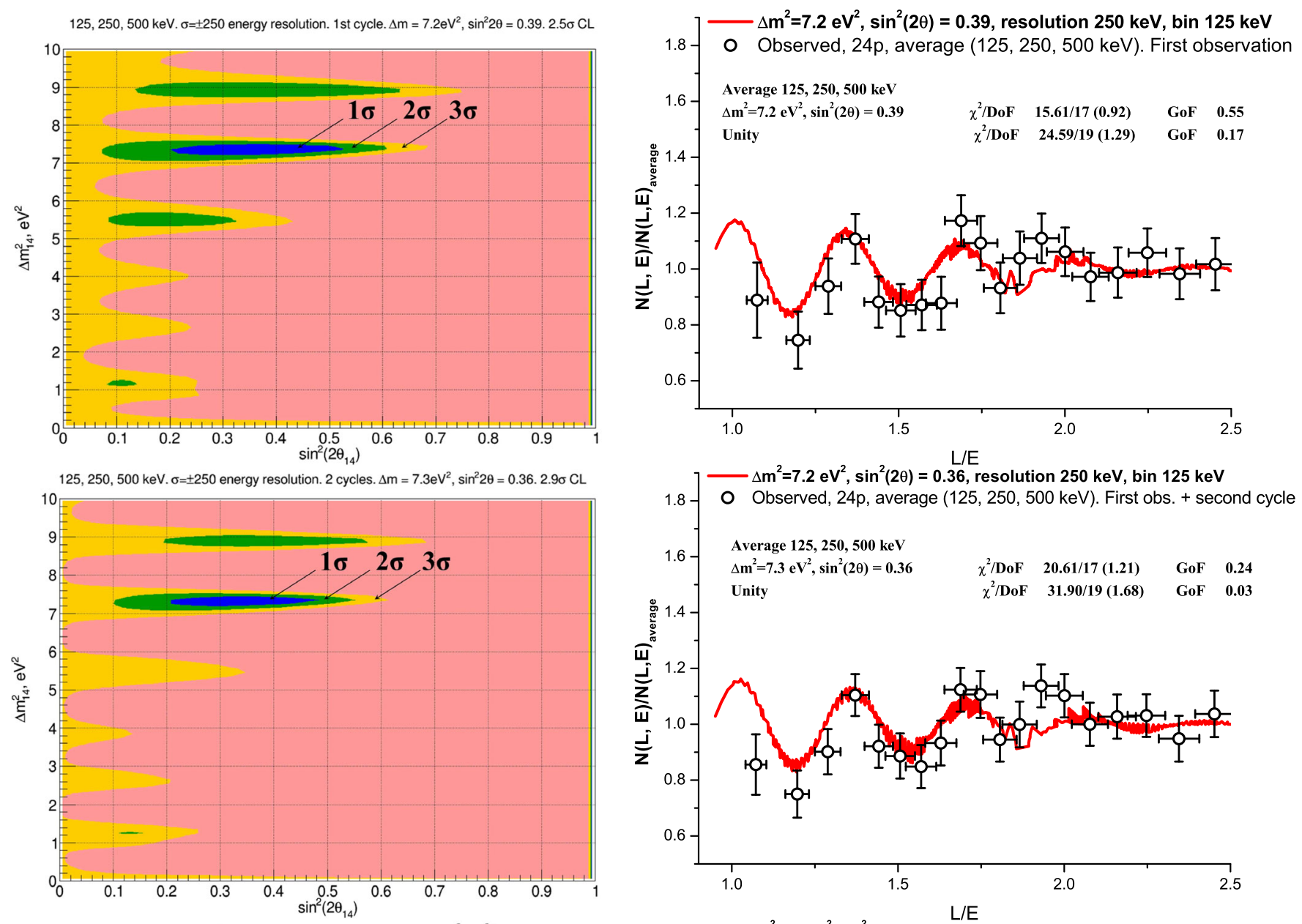

$125,250,500$ keV. $\sigma= \pm 250$ energy resolution. 3 cycles. $\Delta m=7.2 \mathrm{eV}^{2}, \sin ^{2} 2 \theta=0.36 .2 .3 \sigma \mathrm{CL}$
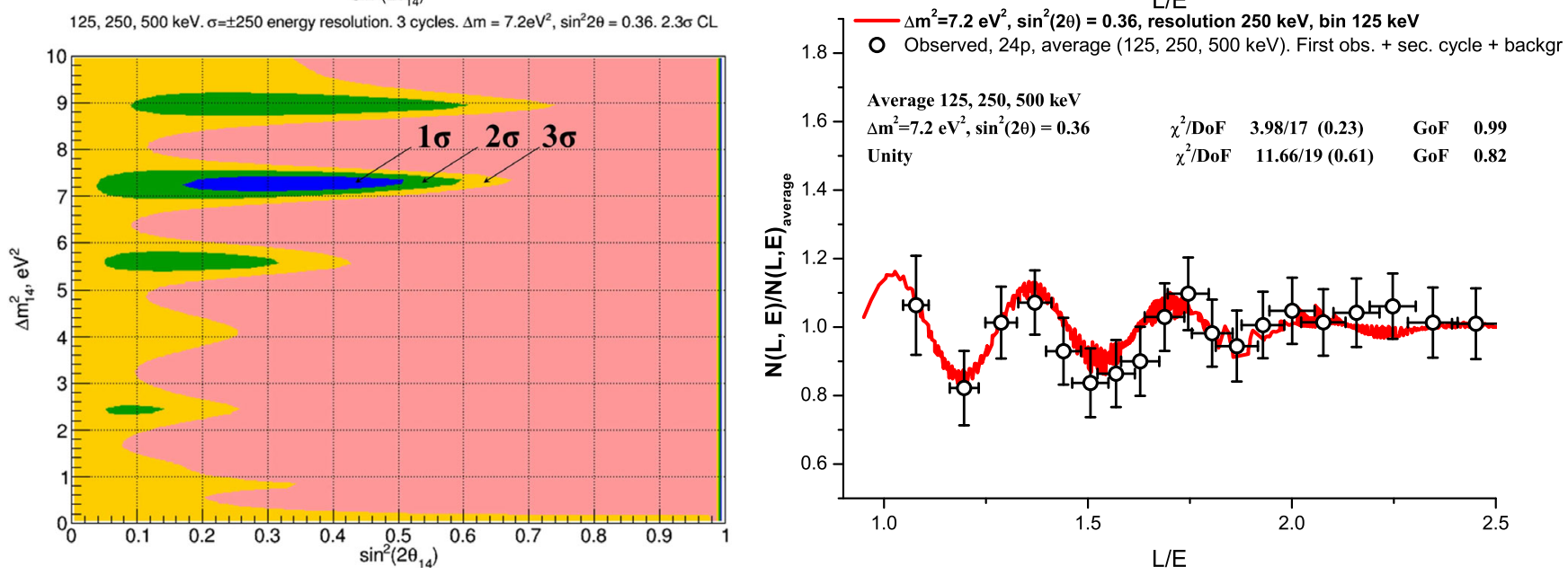

FIG. 44. Top: results of data analysis of the first measurement cycle (I), middle: the results of data analysis of the first measurement cycle together with the second measurement cycle (II), bottom: results of data analysis of the first measurement cycle, with the second measurement cycle and with the third measurement cycle (III). On the left is the central part of the region $\Delta m_{14}^{2}, \sin ^{2} 2 \theta_{14}$ and on the right is the oscillation curve. 
TABLE III. Oscillation parameters obtained in first cycle, first and second cycles and for the three cycles together.

\begin{tabular}{lccc}
\hline \hline Case & $\begin{array}{c}\text { Best fit oscillation parameters } \\
\Delta m_{14}^{2}, \sin ^{2} 2 \theta_{14}\end{array}$ & $\begin{array}{c}\left.\chi^{2} / \text { d.o.f. (Reduced } \chi^{2}\right) \\
\text { fit w/ and w/o oscillation }\end{array}$ & $\begin{array}{c}\text { Goodness of fit w/ } \\
\text { and w/o oscillation }\end{array}$ \\
\hline I & $\mathbf{7 . 2} \mathbf{e V}^{\mathbf{2}}, \mathbf{0 . 3 9}(\mathbf{2 . 5 \sigma})$ & $\mathbf{1 5 . 6 1 / 1 7}(\mathbf{0 . 9 2}) 24.59 / 19(1.29)$ & 0.550 .17 \\
II & $\mathbf{7 . 3} \mathbf{e V}^{\mathbf{2}} \mathbf{0 . 3 6}(\mathbf{2 . 9 \sigma})$ & $\mathbf{2 0 . 6 1 / 1 7}(\mathbf{1 . 2 1}) 31.90 / 19(1.68)$ & 0.240 .03 \\
III & $\mathbf{7 . 2} \mathbf{e V}^{\mathbf{2}}, \mathbf{0 . 3 6}(\mathbf{2 . 3 \sigma})$ & $\mathbf{3 . 9 8} / \mathbf{1 7}(\mathbf{0 . 2 3}) 11.66 / 19(0.61)$ & 0.990 .82 \\
\hline \hline
\end{tabular}

more than $3 \sigma$. However, in the area, $\Delta \mathrm{m}_{14}^{2}=7.3 \mathrm{eV}^{2}$ and $\sin ^{2} 2 \theta_{14}=0.36 \pm 0.12_{\text {stat }}$, the oscillation effect is observed at $2.9 \sigma$ C.L. Here, we also illustrate the areas of the reactor anomaly and the Gallium anomaly.

The Fig. 44 shows the results of the data analysis of the first measurement cycle (I), the results of the data analysis of the first measurement cycle together with the second measurement cycle (II), and the results of the data analysis of the first measurement cycle, with the second measurement cycle and with the third measurement cycle (III). On the left is the part of the region on the $\Delta m_{14}^{2}, \sin ^{2} 2 \theta_{14}$ plane, and on the right is the oscillation curve. It should be noted, that due to error, an increased goodness of fit criterium is closing to unity, which is natural if the effect exists, but increasing of errors is out of the statistical limitation.

In Table III, we list the oscillation parameters, $\chi^{2} /$ d.o.f. and GoF for all three cases (I, II, and III);

It is important to emphasize that the statistical uncertainty of the parameter $\sin ^{2} 2 \theta_{14}$ decreased after the inclusion of the data of the second measurement cycle, while the C.L. increased. However, if we include in the analysis, additional measurements of the background obtained in the third cycle, the C.L. decrease, and the statistical uncertainty of the parameter $\sin ^{2} 2 \theta_{14}$ increase. It was mentioned above that measurements with short intervals between signal and background measurements are preferable. In this case, the background fluctuations are compensated. If we compare the results of background measurements with the results obtained with the reactor in operation mode, which have a time interval of 1-2 years, then we can get the deviation of the background which exceeds the statistical accuracy. It leads only to an increase, not a decrease, in measurement uncertainty. Therefore, we must focus on the results obtained in the first and second cycles of measurements, $\Delta m_{14}^{2}=7.3 \pm 0.13_{\text {st }} \pm 1.16_{\text {sys }}=7.3 \pm 1.17, \sin ^{2} 2 \theta=0.36 \pm$ $0.12(2.9 \sigma)$.

\section{ANALYSIS OF POSSIBLE SYSTEMATIC EFFECTS}

1. The study of possible systematic effects was performed using a background of fast neutrons created by cosmic rays. In order to study systematic effects, one has to turn off antineutrino flux (turn off the reactor) and perform the same analysis of the collected data. That procedure has sufficient precision since we have collected a significant amount of background data. The result of background measurements analysis for oscillation effect manifestation on the plane $\Delta \mathrm{m}_{14}^{2}$ and $\sin ^{2} 2 \theta_{14}$ is shown in Fig. 45. The data of all measurement cycles are combined.

The results of the background analysis is presented in Fig. 46 (bottom), which shows that the background cannot form the signal observed for the on-off data (Fig. 46 top).

Correlated background (fast neutrons from cosmic rays) slightly depends on distances from the reactor due to the inequality of concrete elements of the building. If, earlier during constructing the $\mathrm{R}$ ratio for the neutrino signal, we introduced a correction for the distance $L^{2}$, now we used the dependence of the background on the distance, shown in Fig. 34.

From Fig. 46, it can be seen that the dependence of the $\mathrm{R}$ ratio on $\mathrm{L} / \mathrm{E}$ satisfies a constant fit with $\chi^{2} /$ d.o.f. $=1.3$, when the oscillation fit gives $\chi^{2} /$ d.o.f. $=6.1$. Therefore, we can conclude that the apparatus does not produce a systematical effect.

It is shown above that it is unreasonable to add corrections into the oscillation curve. Also, the square root of the sum of squares of significant uncertainties of points at the oscillation curve and small uncertainties at the background curve is very close to

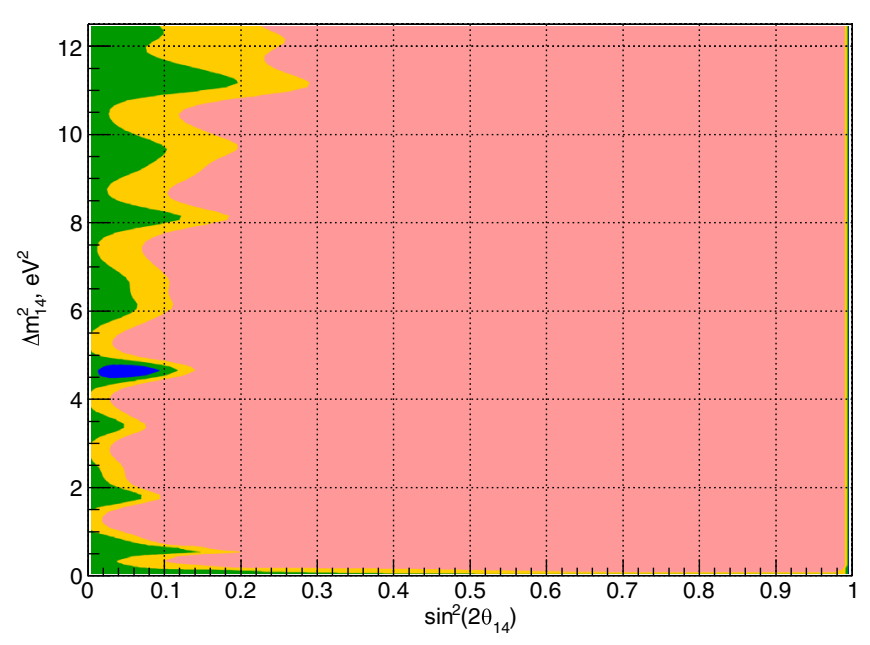

FIG. 45. Data analysis results for the background on the plane $\Delta \mathrm{m}_{14}^{2}$ and $\sin ^{2} 2 \theta_{14}$. 

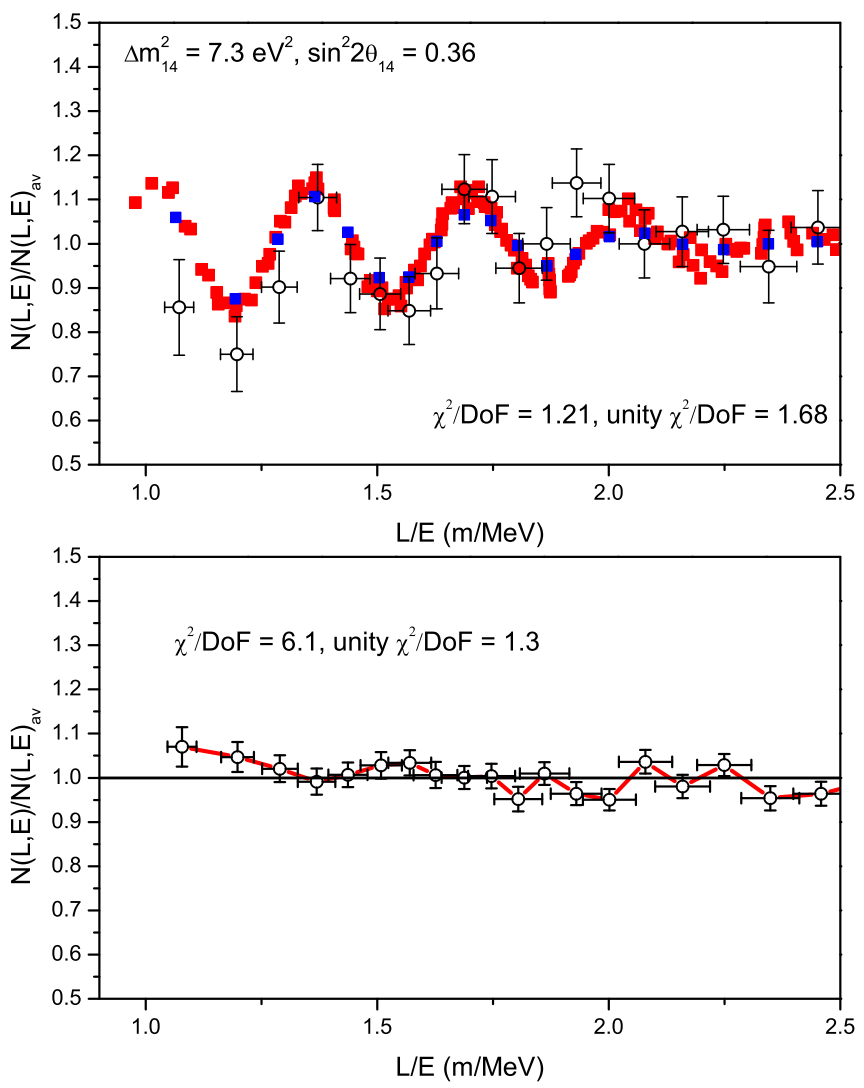

FIG. 46. Comparison of the $\mathrm{R}$ ratio versus $\mathrm{L} / \mathrm{E}$ for the neutrino signal (top) and the $\mathrm{R}$ ratio versus $\mathrm{L} / \mathrm{E}$ for the background (bottom).

the uncertainties at the oscillation curve (deviation is about $2 \%-3 \%$ of the uncertainties at the oscillation curve).

2. In addition, it should be noted that the experimental points should be fitted with such a sinusoidal dependence, which has a maximum at the origin, since the process of oscillations starts from the source. This significantly reduces the set of sinusoids available for fitting. Figure 47 shows the complete curve of the oscillation process starting from the reactor. There is a maximum at zero; from here, the process of oscillations begins.

3. Below we are trying to perform an additional search for systematic effects connected with the correlated background.

We would like to remind that correlated background arise from fast neutrons as a result of elastic and inelastic scattering $n, n^{\prime}$. In the reaction of elastic scattering on hydrogen, a recoil proton appears which imitates the signal from a positron. The reaction of inelastic scattering of fast neutrons occurs on carbon nuclei. The fast neutron excites the carbon nucleus, which deexcites before the neutron is thermalized and captured. This process produces a correlated event similar to the IBD

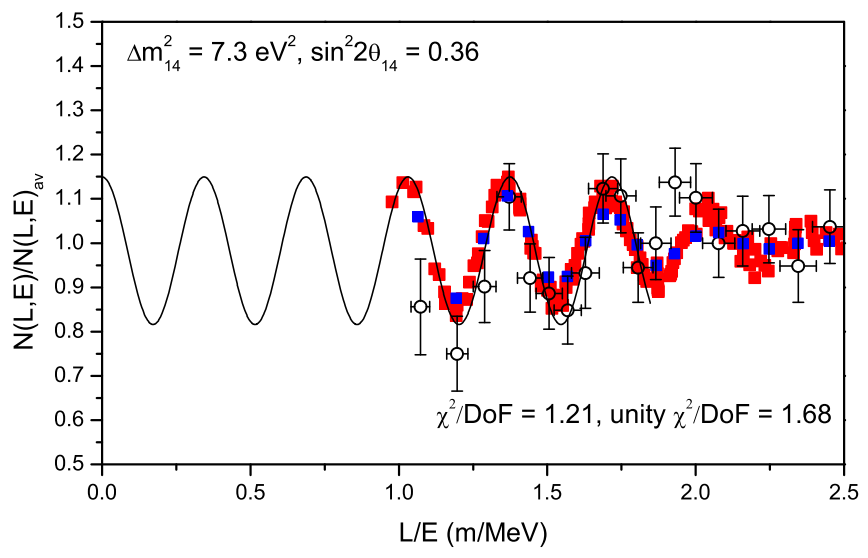

FIG. 47. Complete curve of the oscillation process starting from the reactor core center.

process. This looks rather dangerous for the search for neutrino oscillations. However, it should be clarified that the background spectrum is subtracted when we form the on-off difference signal to detect neutrino events. Again, one may be concerned that this subtraction may not be complete due to the effect of background fluctuations associated with fluctuations in atmospheric pressure and temperature since measurements with the reactor running and with the switched off states occur at different times. However, it is possible to quantify this incomplete compensation of the effect. In our case, the measurements have been carried out about for about 4 years and the atmospheric pressure variations that is $\pm 1.1 \%$ were nicely averaged. Since the reactor on and off operations occurred 87 times, the average contribution of cosmic background fluctuations to the measurement results does not exceed $\pm 0.1 \%$ or approximately, $\pm(0.3 \div 0.5) \%$ with respect to the neutrino signal, the oscillations of which are $\pm(10 \div 15) \%$.

So, background fluctuations uncorrelated with the reactor power do not create a danger of a false oscillation effect. Therefore, it is necessary to investigate the possible change in any parameters, correlated with the reactor power. For example, the temperature in the laboratory increases when the reactor is operating. If in this case, for example, the gain of the PMT changes; this will lead to a shift in the spectra. Irregularities in the background spectrum will be shifted. When the background is subtracted, a difference with a periodic structure appears. This reasoning raises the following concern about the possibility of a false oscillation effect. This requires a quantitative assessment of the possible size of the effect.

The temperature in the laboratory compartment when the reactor was turned on was not observed to be changing within an accuracy of $1^{\circ}-2^{\circ}$. It should 
be noted that the detector, together with a part of the electronic equipment, is inside a passive shield weighing 60 tons. This protection functions almost as a cryostat. The temperature coefficient of voltage stability at the PMT is $0.23 \mathrm{~V} /{ }^{\circ}$. These studies were carried out in a special stand with a temperature change of $20^{\circ}$. The change in the PMT Hamamatsu $\mathrm{R} 5912$ coefficient is $0.5 \%$ per degree; therefore, the spectrum shift in the $6000 \mathrm{keV}$ region is $30-60 \mathrm{keV}$. Finally, in another special stand, the temperature stability of the entire system, including the PMT and the scintillator, had been checked. The temperature range was also $20^{\circ}$, and the temperature stability was $1 \%$ per degree. Thus, the possible shift of the spectrum in the region of $6000 \mathrm{keV}$ is $60-120 \mathrm{keV}$.

To test the possibility of generating a false oscillation effect, data processing was simulated, taking into account such a bias. This processing showed that a shift of the spectrum in the region of $6000 \mathrm{keV}$ by $120 \mathrm{keV}$ is not dangerous and does not simulate the formation of a false oscillation effect.

4. The next question is an influence of unequal efficiency of neutrino events registration in different detector rows. It should be noted that in this case, we are talking about the efficiency of correlated signals detection which involves several sections at once, as shown in Fig. 32 in Sec. XIII on computer simulation of full-scale detector. Registration efficiency of the section depends on its position. However, each section moves together with its environment and its efficiency does not depend on the position of the detector. Here, it is important to recall that we use the method of relative measurements with the movement of the detector, and in addition, the registration efficiency for each section is canceled in $R_{i k}^{\exp }$ ratio. If all sections collected data at every point, then no questions should arise at all. However, measurements by edge sections are not reproduced by central sections. As will be shown in the future, no such influence is observed beyond statistical accuracy. Nevertheless, a direct analysis of the possible impact of inequality among sections efficiencies on the observation of the oscillation process would be conducted.

The determination of the efficiency of individual sections should be done experimentally. In fact, the efficiency of the individual rows of the detector, which includes five sections located at the same distance, is needed.

For the experimental determination of rows efficiency with respect to correlated signals, the background of fast neutrons of cosmic origin can be used. In principle, this requires a source that irradiates the entire detector uniformly, like a neutrino flux. The background of fast neutrons of cosmic origin is uniform as shown in Fig. 5 in Sec. V. However, a self-screening process occurs in the detector, so the first and last rows of the detector show a higher count rate. The dependence of the count rate on the number of the detector row is shown in Fig. 48 at the top.

This dependency was obtained in the following measurements. The distances of detector movements correspond to section size $(23.5 \mathrm{~cm})$. All movements are controlled with laser distance measurer. The measurements were carried out at ten detector positions in the way that the same distance from the reactor is measured with various detector rows. Spectra measured with various rows at same distance are averaged afterwards.

The average distribution of prompt signal counts obtained in background measurements during the whole period of reactor stop is shown in Fig. 48 (top). We should remind that the first and last rows are not used for obtaining the final dependence on L/E. They are used as active and passive shielding from neutrons. They screen central fiducial part of the detector. It was mentioned before that the direct rate (not correlated) of fast neutrons induced by
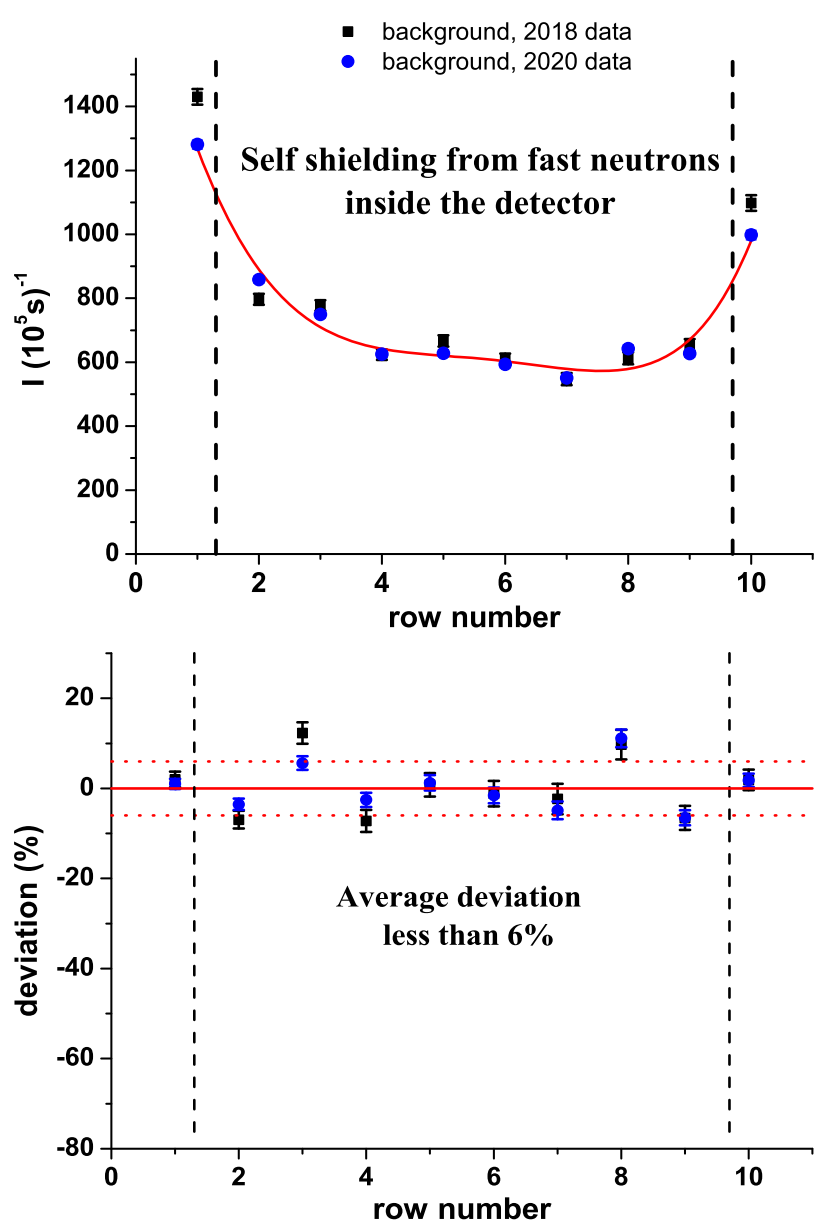

FIG. 48. Average distribution of correlated background prompt signals in detector over all positions (top). Deviation average distribution of prompt signals from profile. Profile was caused by inhomogeneity of fast neutrons background in the lab room (bottom). 
cosmic background inside passive shielding is uniform. However, for the correlated background, some gradient within the 6-12 $\mathrm{m}$ distance range is observed, which can occur due to concrete structures of the building (see Fig. 2).

The red line in Fig. 48 (top) is the profile of the fast neutron distribution due to self-screening effect. The deviation of counts from an average value can be interpreted as the difference in efficiency of different rows. The mean value of the deviation is $\sim 6 \%$ (Fig. 48 bottom). However, different rows of the detector perform measurements at the same distance, and it is the reason why the average efficiency plays the role for that particular distance.

To consider how differences in rows efficiencies affect the final results, one must take into account that averaging of spectra obtained with various rows at the same distance. In that approach, the square deviation from the mean value is $\sim 1.8 \%$, as shown in Fig. 49 (top). It indicates that the detector inhomogeneity cannot be the origin of oscillation effect. Nevertheless, it is expedient to make MC simulations for complete clarity of the situation. Results of these simulations are on the Fig. 49 (second).

It can be seen, that considering deviations in the efficiency of detecting correlated events at different distances did not affect the effect of oscillations (see Fig. 49 third and fourth). This situation can be explained since the effect of oscillations is resonant and successfully survives in the presence of noise.

We can summarize our analysis of systematic uncertainties by concluding that these uncertainties cannot explain the observed oscillation effect.

Indeed, after averaging over groups of eight points each, the effect of noise is significantly suppressed (see Fig. 49, fourth) because the characteristic noise frequency is higher than the oscillation curve frequency. The amplitude of noise fluctuations is no more than $1 \%$ whereas the amplitude of the oscillation curve is $15 \%$.

\section{SYSTEMATIC ERRORS OF THE EXPERIMENT}

One of possible systematic errors of the oscillation parameter $\Delta \mathrm{m}_{14}^{2}$ is determined by the accuracy of the energy calibration of the detector. The relative accuracy of ratio $\mathrm{L} / \mathrm{E}$ is determined by the relative accuracy of the measurements of energy, because the relative accuracy of the measurements of distance is significantly better. The relative accuracy of measurements of energy in the most statistically significant area of the measured neutrino spectrum 3-4 MeV is 5\%. Hence, the possible systematic error of parameter $\Delta \mathrm{m}_{14}^{2}$ is $0.6 \mathrm{eV}^{2}, \delta\left(\Delta m^{2}\right)_{\text {syst } 1} \approx 0.6 \mathrm{eV}^{2}$.

Another systematic error of parameter $\Delta \mathrm{m}_{14}^{2}$ can occur in data analysis performed with $\chi^{2}$ method because of additional regions around the optimal value $\Delta \mathrm{m}_{14}^{2} \approx 7.3 \mathrm{eV}^{2}$. In particular, the closest regions have values $5.2 \mathrm{eV}^{2}$ and
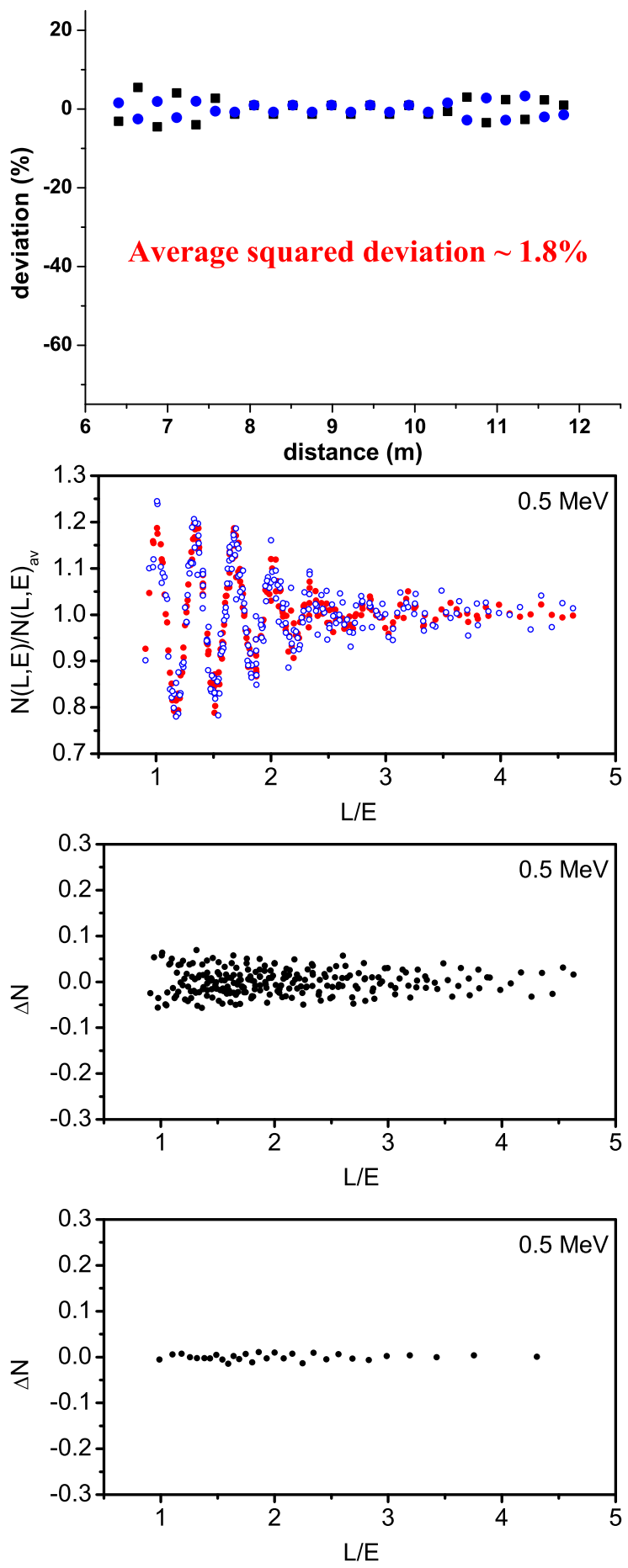

FIG. 49. Top: deviation of counts of correlated background of each distance from the reactor after averaging over rows from the mean value. Second: MS modeling of the oscillation effect considering deviations of the detection efficiencies for correlated events at different distances. Red dots: oscillation effect ignoring the influence of deviations of the correlated events registration efficiencies at different distances; blue dots: oscillation effect considering this influence. Third: difference between red and blue dots and fourth is sum by 8 of the third one. 
$8.8 \mathrm{eV}^{2}$, as can be seen from the Fig. 43. However, contribution of the satellite at $5.2 \mathrm{eV}^{2}$ is significantly smaller. Therefore, the possible systematic error of $\Delta \mathrm{m}_{14}^{2}$ can be estimated as $\delta\left(\Delta m^{2}\right)_{\text {syst2 }} \approx 1.0 \mathrm{eV}^{2}$. Finally, the obtained value of difference squared masses is

$\Delta m_{14}^{2}=\left(7.3 \pm 0.13_{\text {st }} \pm 1.16_{\text {syst }}\right) \mathrm{eV}^{2}=(7.3 \pm 1.17) \mathrm{eV}^{2}$.

The statistical error of parameter $\sin ^{2} 2 \theta_{14}$ can occur in the calculation of the optimal value of $\sin ^{2} 2 \theta_{14}$ using the $\chi^{2}$ method,

$$
\sin ^{2} 2 \theta=0.36 \pm 0.12_{\text {stat }}
$$

\section{THE DEPENDENCE OF THE REACTOR ANTINEUTRINO FLUX ON DISTANCE IN RANGE 6-12 METERS}

Results of measurements of the difference in counting rates of neutrino events (reactor on-off) are shown in Figs. 50-52, as the dependence of antineutrino flux on the distance to the reactor core. Fit of an experimental dependence with the law $\mathrm{A} / \mathrm{L}^{2}$ yields the following result. Goodness of that fit is $22 \%$. However, there is an indication that in the range 9-12 $\mathrm{m}$, the rate is smaller than in the range 6-9 $\mathrm{m}$, and the curve that fitting the obtained points resemble the oscillation curve with period $6-8 \mathrm{~m}$, which corresponds to $\Delta m_{14}^{2}=1.2 \mathrm{eV}^{2}$. One can find the corresponding small green spot on the plane $\Delta m_{14}^{2}, \sin ^{2} 2 \theta_{14}$ (see Fig. 43 middle). However, this result is inconclusive because the deviation is within statistical uncertainties. Moreover, it can be the result of the fact that in the second measurement cycle the results were obtained mainly for the short distances. Finally, the background in range $9-12 \mathrm{~m}$ is

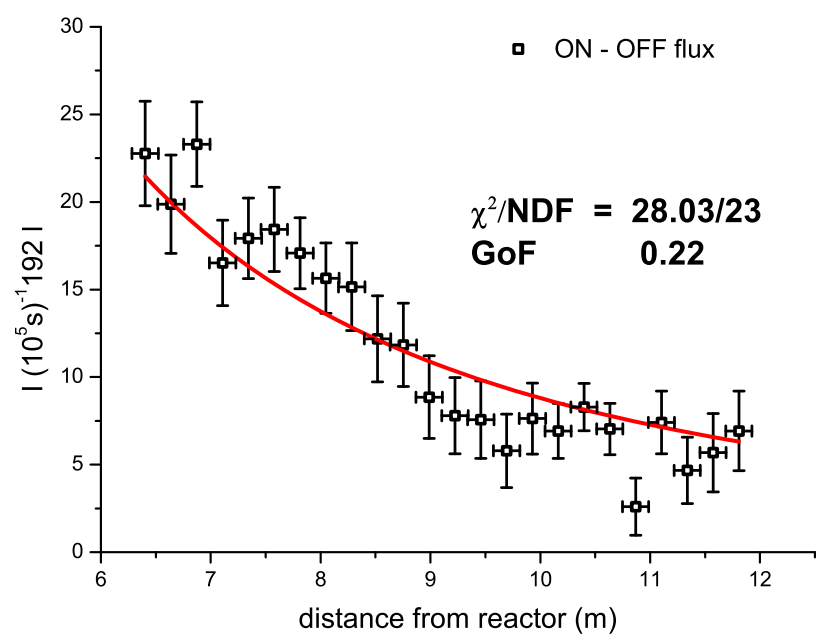

FIG. 50. Dependence of antineutrino flux on the distance to the reactor core-direct measurements with subtracted background.

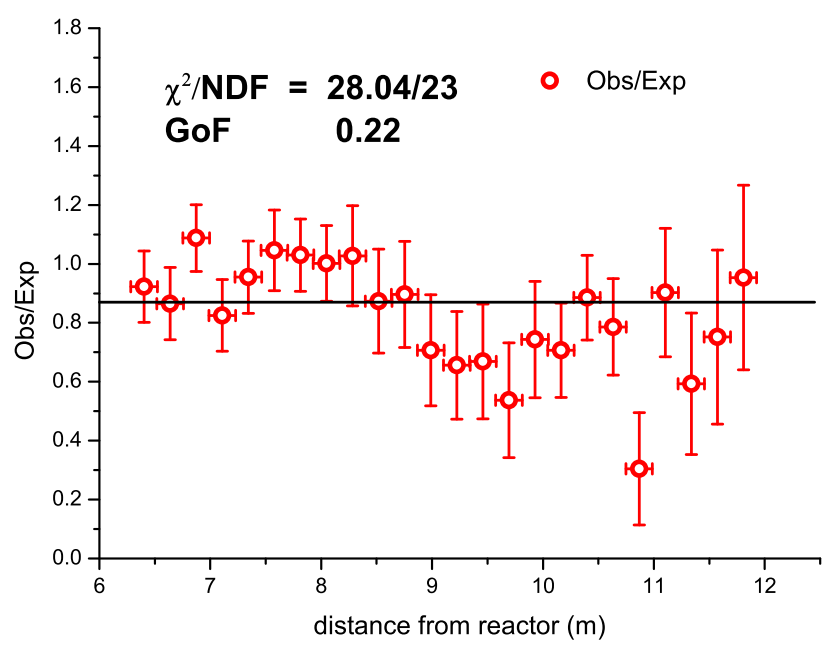

FIG. 51. Representation of experimental results in form of dependence of antineutrino flux on the distance to the reactor core normalized with the law $\mathrm{A} / \mathrm{L}^{2}$.

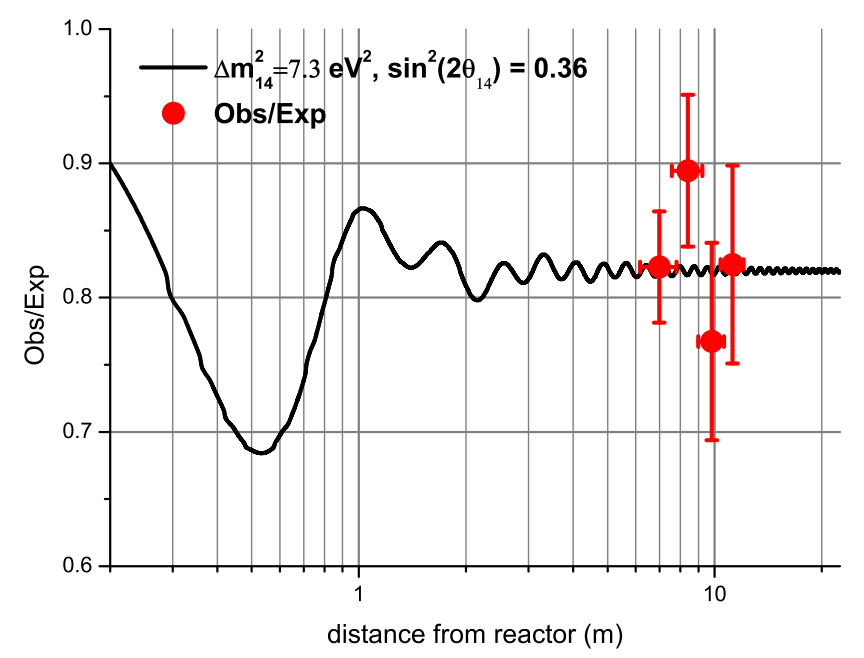

FIG. 52. Oscillation curve and experimental results in range 6-12 m.

partially suppressed by the concrete celling of the building. The statistical fluctuation is the most unbiased of all the mentioned hypotheses. Corrections for finite size of reactor core and detector sections are negligible $-0.3 \%$, and correction for the difference between the detector movement axis and direction to center of reactor core is also negligible—about $0.6 \%$.

\section{MC MODEL OF THE EXPERIMENT WHICH TAKING INTO CONSIDERATION OBTAINED STATISTICAL ACCURACY}

In Sec. XVIII, we presented the simulation of the experiment without taking into account the background conditions. In this section, we present the simulation of the experiment taking into account the background conditions 
observed in the experiment and the energy dependence of the energy resolution of the detector.

In the Monte Carlo model, as before, an antineutrino source with a reactor core size of $42 \times 42 \times 35 \mathrm{~cm}$ and an antineutrino detector consisting of 50 sections with geometric dimensions $22.5 \times 22.5 \times 75 \mathrm{~cm}$ were considered. Sections in one row have been combined. Antineutrinos were detected at all 24 distances from $6.5 \mathrm{~m}$ to $12 \mathrm{~m}$ with a row size step of $22.5 \mathrm{~cm}$. The antineutrino spectrum was set in accordance with the antineutrino spectrum for ${ }^{235} \mathrm{U}$, although this is not important since we used the method of relative measurements.

The number of simulated events corresponded to the experimental data (on-off), accumulated in the experiment at different distances. The simulated count dispersion was in accordance with the statistical error of the experiment, so the background was taken into account. In this case, we used statistical uncertainties obtained in the first and second cycle of measurements. Data collected during long time reactor off period were not included in the analysis. Thus, the experimental matrix $N_{i k}=N\left(E_{i}^{\nu}, L_{k}\right)$ was modeled. The on-off neutrino count was simulated for two cases with and without oscillations. For the case with oscillations, we used the parameters obtained as a result of processing the experimental data $-\Delta m_{14}^{2}=7.3 \mathrm{eV}^{2}, \sin ^{2} 2 \theta_{14}=0.36$.

The final goal was to reproduce the experiment repeatedly and to form the distribution for the two mentioned cases by the data averaging method. To obtain an oscillatory curve, each of the simulated experiments was analyzed using the same processing scheme as in the real experiment. Each time the analysis was carried out with the hypothesis of oscillations and with the hypothesis without oscillations. Thus, it became clear which of the hypotheses is better suited for each of the experiments performed. For this, the value $\chi^{2}$ was calculated each time to fit the result with the oscillation curve $\chi_{\text {sin }}^{2}$ or by the constant $\chi_{\text {const }}^{2}$. In general, $5 \times 10^{6}$ experiments were carried out for the case with oscillations and the same number without oscillations.

Figure 53 shows the three-dimensional distribution of the reduced $\chi^{2}$ values on the plane $\left(\chi_{\text {const }}^{2}, \chi_{\text {sin }}^{2}\right)$. It should be noted that the values $\chi_{\text {const }}^{2}, \chi_{\sin }^{2}$ are correlated, since they were obtained from the same simulation but with different processing hypothesis. It is quite obvious that for experiments without oscillations, the optimal value of $\chi_{\text {const }}^{2} \approx 1$ should be obtained when processing with a hypothesis without oscillations. While when processing data with a hypothesis with oscillations, the optimal value will be $\chi_{\sin }^{2}>1$. For experiments with oscillations, the situation will be similar, but vice versa. This is optimal for $\chi_{\sin }^{2} \approx 1$ and $\chi_{\text {const }}^{2}>1$. To generate these distributions, 17 degrees of freedom and 19 degrees of freedom were used for analysis of simulations without oscillation and with oscillation, respectively. It should be noted that the distribution of results in the experiment was broadened (see Sec. XII), and the simulation takes it into account.
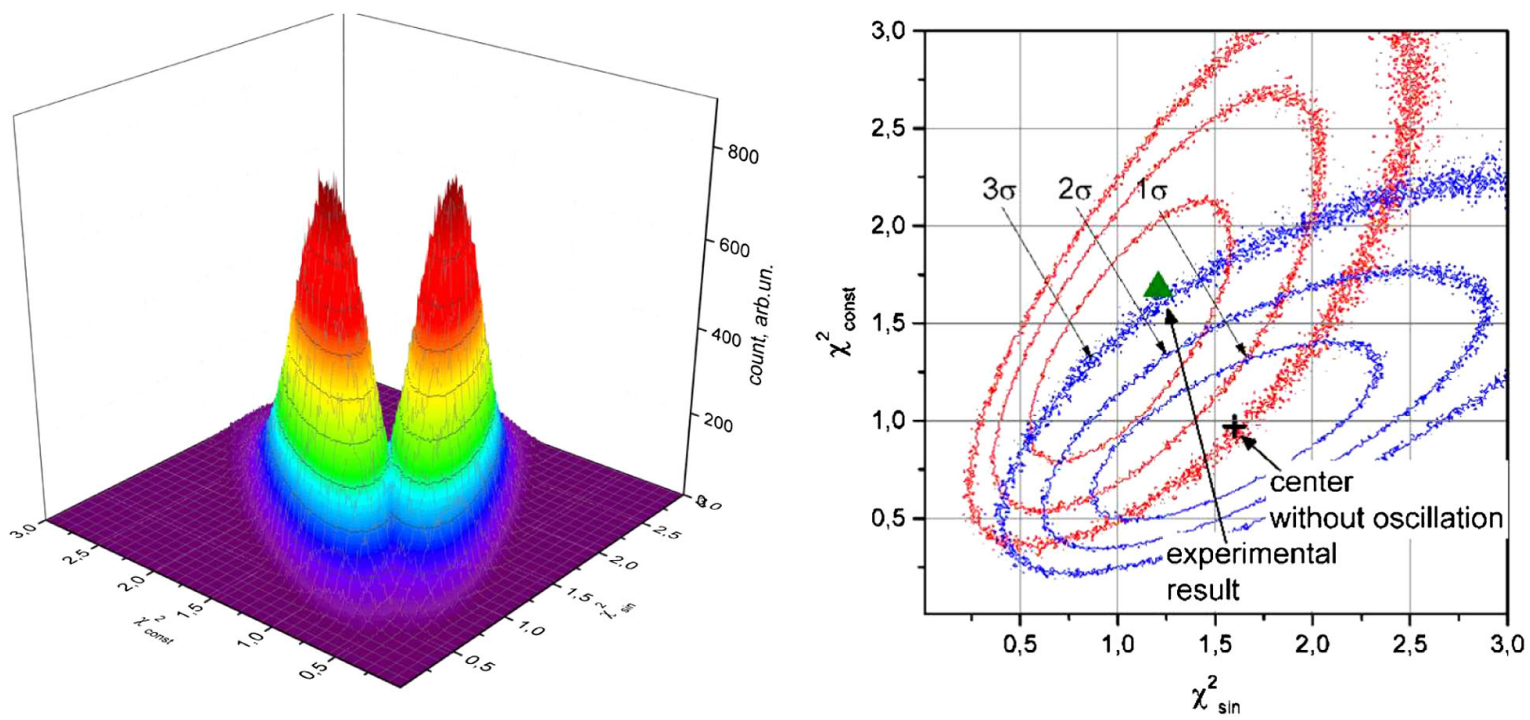

FIG. 53. On the left, the distribution of the reduced $\chi^{2}$ on the plane $\left(\chi_{\text {const }}^{2}, \chi_{\text {sin }}^{2}\right)$ is shown for modeling with accuracy according to the experimental statistics and with a background level corresponding to the experimental background. Two cases were simulated with the hypothesis of oscillations and with the hypothesis without oscillations. For each case, an analysis was carried out with the hypothesis of oscillations and with the hypothesis without oscillations. On the right is the same picture, where the center of the distribution without oscillations (black cross), and the deviation from this center of the experimental result (green triangle-first and second cycle) are indicated. The result of the analysis of the experimental data when processed with the hypothesis with and without oscillations gives $\chi_{\sin }^{2}=1.21$ or 20.6 for 17 degrees of freedom and $\chi_{\text {const }}^{2}=1.68$, or 31.9 for 19 degrees of freedom, respectively, and $\Delta \chi^{2}=11.3$. Distribution contours with $1 \sigma, 2 \sigma$, and $3 \sigma$ are marked. The deviation of the experimental result from the center of the distribution without oscillations is approximately $3 \sigma$. 
The result of the simulation reveals that value $\Delta \chi^{2}>11.3$ corresponds to the probability of false signal of oscillation $2.6 \times 10^{-4}$, i.e., confidence level of oscillation effect with $\Delta m_{14}^{2}=7.3 \mathrm{eV}^{2}, \sin ^{2} 2 \theta_{14}=0.36$ is 3.6 $\sigma$. However, the presented analysis of the simulated data was done with fixed oscillation parameters. More rigorous analysis for possible accidental false oscillation effect could be done with free oscillation parameters.

As shown in $[37,38]$, in order to obtain a more accurate estimation of statistical significance, one should use a MCsimulation based statistical analysis. This method includes usage of free oscillation parameters, and we applied it to analyze the results of the Neutrino-4 experiment. Within this approach, we simulate spectra of prompt signals in the neutrino detector at different distances from the reactor using the no oscillation hypothesis. The dispersion of simulated values was determined by the statistical accuracy of the $N_{i k}$ matrix of the experiment, i.e., by $\Delta N_{i k}$. Spectra of each simulation processed like experimental spectra, using the method described in Sec. XVII, i.e., by constructing the matrix R. The simulation of values of the $N_{i k}$ matrix seems more accurate because such a simulation better represents the order of data processing in our experiment. However, the calculations proves that the simpler approach based on direct usage of the matrix $\mathrm{R}$ gives a similar final result. The values of $R_{i k}$ obtained from simulated values of $N_{i k}$ follows the normal distribution. We performed $1.5 \times 10^{4}$ simulations and the obtained distribution of $T=\chi_{\text {null }}^{2}-\chi_{\text {best fit }}^{2}$, where $\chi_{\text {null }}^{2}=\chi^{2}(0,0)$ chi-squared for the null hypothesis, $\chi_{\text {best fit }}^{2}=\chi^{2}\left(\sin ^{2} 2 \theta\right.$, $\left.\Delta m^{2}\right)$-minimal chi-squared corresponding to some oscillation parameters, is shown in Fig. 54.

This distribution is different from $\chi^{2}$ with 2 degrees of freedom, which Wilks's theorem claims. However, the fraction of simulated results with $T \geq 11.3$ is only 0.0075 ,

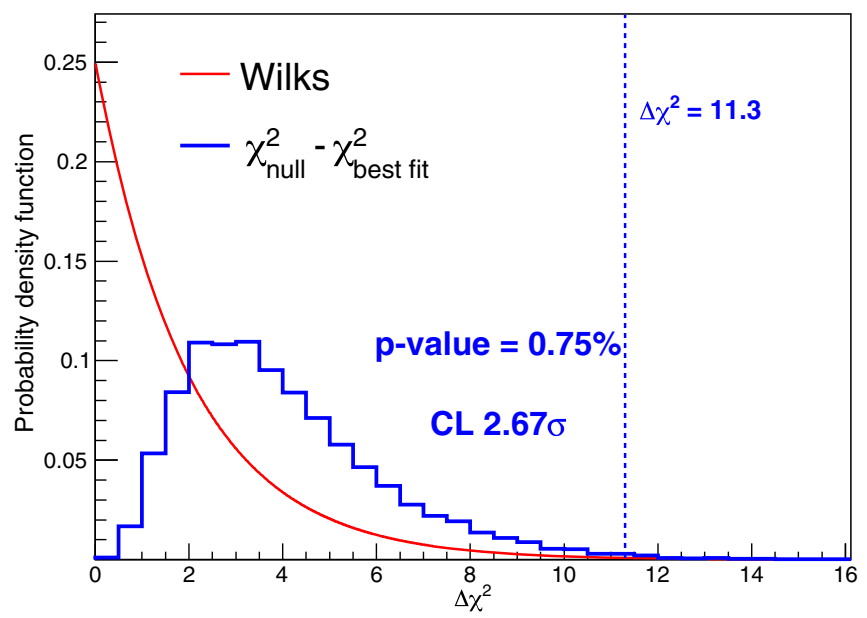

FIG. 54. $T$ distribution for MC based approach to the statistical analysis (blue line) and $\chi^{2}$ with 2 degrees of freedom function, which is claimed by Wilks's theorem. which corresponds to $2.7 \sigma$ C.L. Therefore, using this stricter criterion to estimate the confidence level in the case of the oscillation search in the Neutrino-4 experiment, we can make a conclusion about the observation of neutrino oscillation into a sterile state at $2.7 \sigma$ C.L.

We plan to continue data taking. However, it is quite obvious that the continuation of the collection of data without improving the installation is not advisable. It is necessary to suppress the background and improve the sensitivity of the setup. We are planning to improve the existing installation, and, in addition, we are planning to create a second neutrino laboratory at the SM-3 reactor with a detector that is 3 times more sensitive.

\section{ANALISYS OF THE GENERAL SITUATION CONCERNING EXPERIMENTAL ATTEMPTS OF STERILE NEITRINO SEARCH}

Despite the fact that the current confidence level is insufficient to make a conclusion about the existence of the oscillation effect, it is reasonable to 1 ) check if it contradicts other results of experiments on the search for a sterile neutrino and analyze the agreement with other experiments on the search for a sterile neutrino 2) analyze what experiments can confirm the obtained result. We do not aim to review other experiments. We are only concerned about the clear disagreement with other experiments, which can impugn results of our experiment at the current step. That is a very important part, because the issue in question is the discovery of a new particle outside the Standard Model. The detailed analysis was performed in the work [36]. Here, we would like to present a short summary.

First of all, we should notice that this topic was investigated in many different experiments. In the sector of the measurements at the reactors, there are experiments performed at nuclear power plants and research reactors.

In experiments on the nuclear power plants DANSS [17], NEOS [18] sensitivity to the identification of the effect of oscillations with large $\Delta \mathrm{m}_{14}^{2}$ is considerably suppressed because of the big sizes of an active zone. The experiments PROSPECT [19] and STEREO [20] have good sensitivity to large values of $\Delta \mathrm{m}_{14}^{2}$, but they have started data collection later, so possibly it will confirm or close our result in the future.

The oscillations obtained in the Neutrino-4 experiment gives an oscillation parameter $\sin ^{2} 2 \theta_{14} \approx 0.36 \pm$ $0.12(2.9 \sigma)$. The neutrino deficiency called gallium anomaly (GA) [8,9] gives the oscillation parameter $\sin ^{2} 2 \theta_{14} \approx 0.32 \pm 0.10(3.2 \sigma)$. The result of reactor antineutrino anomaly (RAA) [29-32] measurements is $\sin ^{2} 2 \theta_{14} \approx 0.13 \pm 0.05(2.6 \sigma)$. Combination of these results gives an estimation for mixing angle $\sin ^{2} 2 \theta_{14} \approx$ $0.19 \pm 0.04(4.6 \sigma)$.

The restrictions on the mixing angle, which can be obtained from measurements of reactor anomaly and 
oscillations of the solar neutrinos, are at level $\sin ^{2} 2 \theta_{14} \approx$ $0.1-0.2$ and contradict our result and the estimation obtained in measurements of gallium anomaly $\sin ^{2} 2 \theta_{14} \approx$ $0.3-0.4$. However, the discrepancy between Neutrino-4 and the reactor antineutrino anomaly is currently at $1.7 \sigma$ level. Besides, the systematic uncertainties of the reactor and solar calculations remain questionable.

The values of oscillation parameters obtained in the Neutrino- 4 experiment can be used to estimate the mass of the electron antineutrino, using general formulas for the neutrino model $[39,40]$ with an extension to the $3+1$ model.

Limitations on the sum of the mass of active neutrinos $\sum m_{\nu}=m_{1}+m_{2}+m_{3}$ from cosmology are in the range $0.54 \div 0.11 \mathrm{eV}$ [41]. At the same time, knowing that $\Delta m_{14}^{2} \approx 7.3 \mathrm{eV}^{2}$, it is possible to consider that $m_{4}^{2} \approx 7.3 \mathrm{eV}^{2}$, and $m_{1}^{2}, m_{2}^{2}, m_{3}^{2} \ll m_{4}^{2}$. Thus, the effective mass of the electron neutrino can be calculated by the formula: $m_{\nu_{e}}^{\text {eff }} \approx \sqrt{m_{4}^{2}\left|U_{e 4}\right|^{2}} \approx \frac{1}{2} \sqrt{m_{4}^{2} \sin ^{2} 2 \theta_{14}}$.

It is necessary to make a little discussion here in connection with the known restrictions on the number of types of neutrinos and on the sum of the masses of active neutrinos from cosmology. Depending on the scale of masses, sterile neutrinos can influence the evolution of the Universe and be responsible for the baryonic asymmetry of the Universe and the phenomenon of dark matter [42]. However, the existence of the sterile neutrinos with a low mass and mixing angle does not have a significant effect on cosmology [42]. Such sterile neutrinos practically do not thermalize in the primary plasma and leave it at an early stage.

Considering the facts mentioned above, we can estimate the sterile neutrino mass $m_{4}=(2.70 \pm 0.22) \mathrm{eV}$. In the case of the parameter $\sin ^{2} 2 \theta_{14} \approx 0.19 \pm 0.04(4.6 \sigma)$ obtained combining the results of the Neutrino- 4 experiment and results of the Gallium anomaly measurements and more importantly using the value $\Delta m_{14}^{2} \approx(7.3 \pm 1.17) \mathrm{eV}^{2}$ obtained for the first time in the Neutrino-4 experiment, we can make an estimation of the electron neutrino mass: $m_{\nu_{e}}^{\text {eff }}=(0.59 \pm 0.11) \mathrm{eV}$. The obtained neutrino mass does not contradict the restriction on neutrino mass $m_{\nu_{e}}^{\text {eff }} \leq 1.1 \mathrm{eV}$ (C.L. 90\%) obtained in the KATRIN experiment [43].

In experiments for neutrinoless double beta decay, the Majorana neutrino mass is determined by the following expression: $m(0 \nu \beta \beta)=\sum_{i=1}^{4}\left|U_{e i}\right|^{2} m_{i}$ This expression for the model $3+1$ and with $m_{1}, m_{2}, m_{3} \ll m_{4}$ assumption can be simplified: $m(0 \nu \beta \beta) \approx m_{4} U_{14}^{2}$. The averaged numerical value which includes the results of the Neutrino-4 and other experiments is $m(0 \nu \beta \beta)=(0.13 \pm$ $0.04) \mathrm{eV}$. The best restrictions on the Majorana mass were obtained in the GERDA experiment [44]. The upper limit is $m_{\beta \beta}<$ [0.80-0.182] eV. Therefore, our result does not contradict the results of the experiments on search for neutrinoless double beta decay.
The IceCube experiment and accelerator experiments LSND [1] and MiniBooNE [2] have the results that not only allow the sterile neutrino with a parameter near $\Delta \mathrm{m}_{14}^{2} \approx 7.3 \mathrm{eV}^{2}$ to exist, but even can be considered as an indication on that possibility as shown in Ref. [38].

\section{CONCLUSION}

This work presents the steps of creating the experiment Neutrino-4 from the first preliminary experiments to the obtained result. The first results were published in works [24-28]. Here we discuss the data collected over 5 years to the present day. The first declaration of the observing the oscillation effect at the $3.5 \sigma$ level was published in the work [28]. Further measurements confirmed this result with statistical C.L. $3.2 \sigma$, but taking into account possible systematics, we reduce the C.L. down to $2.9 \sigma$. More accurate statistical analysis gave stricter confidence level at 2.7 $\sigma$. In June 2019, the SM-3 reactor was stopped for renovation. However, the background has been measured from July 2019 till October 2020. We tried to use additional background measurements, but it leads to increasing of statistical error due to uncompensated background fluctuations during long-term measurements.

As a result of the data analysis described in this work, we were able to estimate the actual energy resolution of the detector. Using the obtained energy resolution, we repeated the analysis of measurement results for first and second measurement cycles. The results are presented below,

$$
\begin{aligned}
\Delta m_{14}^{2} & =7.30 \pm 0.13_{\text {st }} \pm 1.16_{\text {syst }}=7.30 \pm 1.17 . \\
\sin ^{2} 2 \theta & =0.36 \pm 0.12_{\text {stat }} .
\end{aligned}
$$

We plan to improve the current experimental facility of the Neutrino- 4 experiment and also create the second neutrino laboratory at the SM-3 reactor which will be equipped with a 3 times more sensitive detector [45]. As a result, we will be able to obtain a final answer for the question of existence of the sterile neutrino with parameter $\Delta \mathrm{m}_{14}^{2}$ near the $7 \mathrm{eV}^{2}$.

\section{ACKNOWLEDGEMENTS}

The authors are grateful to the Russian Science Foundation for support under Contract No. 20-12-00079. Authors are grateful to M. V. Danilov, V. B. Brudanin, V. G. Egorov, Y. Kamyshkov, V. A. Shegelsky, V. V. Sinev, D. S. Gorbunov, and especially to Y.G. Kudenko for beneficial discussions. Also, the authors would like to thank C. Rubbia for useful questions. The delivery of the scintillator from the laboratory headed by Professor Jun Cao (Institute of High Energy Physics, Beijing, China) has made a considerable contribution to this research. 
[1] A. Aguilar et al. (LSND Collaboration), Phys. Rev. D 64, 112007 (2001).

[2] A. Aguilar-Arevalo et al. (MiniBooNE Collaboration), Phys. Rev. Lett. 110, 161801 (2013).

[3] B. Armbruster et al. (KARMEN Collaboration), Phys. Rev. D 65, 112001 (2002).

[4] P. Astier et al. (NOMAD Collaboration), Phys. Lett. B 570, 19 (2003).

[5] M. Antonello et al. Eur. Phys. J. C 73, 2345 (2013).

[6] L. Borodovsky et al. Phys. Rev. Lett. 68, 274 (1992).

[7] N. Agafonova et al. (OPERA Collaboration), J. High Energy Phys. 07 (2013) 004.

[8] W. Hampel et al. (GALLEX Collaboration), Phys. Lett. B 420, 114 (1998).

[9] J. Abdurashitov et al. (SAGE Collaboration), Phys. Rev. C 59, 2246 (1999).

[10] Y. Declais et al. Phys. Lett. B 338, 383 (1994).

[11] B. Achkar et al. Nucl. Phys. B434, 503 (1995).

[12] G. S. Vidyakin et al. JETP Lett. 59, 390 (1994), http:// jetpletters.ru/ps/1307/article_19752.shtml.

[13] A. A. Kuvshinnikov et al. JETP Lett. 54, 259 (1991), http:// jetpletters.ru/ps/1248/article_18875.shtml.

[14] G. Zacek et al. Phys. Rev. D 34, 2621 (1986).

[15] Z. D. Greenwood et al. Phys. Rev. D 53, 6054 (1996).

[16] A. Hoummada, S. Lazrak Mikou, M. Avenier, G. Bagieu, J. F. Cavaignac, and Dy. Holm Koang, Appl. Radiat. Isot. 46, 449 (1995).

[17] I. Alekseev et al. (DANSS Collaboration), Phys. Lett. B 787, 56 (2018).

[18] Y. J. Ko, B. R. Kim, J. Y. Kim et al. (NEOS Collaboration), Phys. Rev. Lett. 118, 121802 (2017).

[19] J. Ashenfelter et al. (PROSPECT Collaboration), Phys. Rev. Lett. 121, 251802 (2018).

[20] H. Almazán et al. (STEREO Collaboration), Phys. Rev. D 102, 052002 (2020).

[21] Y. Abreu et al. (SoLid Collaboration), J. Jpn. Inst. Met. 16, P02025 (2021).

[22] V. Barinov, V. Gavrin, V. Gorbachev, D. Gorbunov, and T. Ibragimova, Phys. Rev. D 99, 111702(R) (2019).

[23] M. G. Aartsen et al. (IceCube Collaboration), Phys. Rev. Lett. 117, 071801 (2016).

[24] A. Serebrov et al. (Neutrino-4 Collaboration), Tech. Phys. 60, 1863 (2015).

[25] A. Serebrov et al. (Neutrino-4 Collaboration), JETP 121, 578 (2015).

[26] A. Serebrov et al. (Neutrino-4 Collaboration), Tech. Phys. 60, 1863 (2015).
[27] A. Serebrov et al. (Neutrino-4 Collaboration), arXiv:1708 .00421 .

[28] A. Serebrov et al. (Neutrino-4 Collaboration), Pis'ma Zh. Eksp. Teor. Fiz. 109, 209 (2019) [JETP Lett. 109, 213 (2019)].

[29] T. Mueller et al. Phys. Rev. C 83, 054615 (2011).

[30] G. Mention, M. Fechner, Th. Lasserre, Th. A. Mueller, D. Lhuillier, M. Cribier, and A. Letourneau Phys. Rev. D 83, 073006 (2011).

[31] S. Gariazzo, C. Giunti, M. Laveder, and Y. F. Lie, J. High Energy Phys. 06 (2017), 135.

[32] D. Lhuillier, in Proceedings of the 16th Lomonosov conference, http://nuclphys.sinp.msu.ru/conf/epp10/Lhuillier .pdf.

[33] J.F. Briesmeister, MCNP-A general Monte Carlo n-particle transport code, Version 4C, Technical Report No. LA-13709-M, 2000.

[34] O. S. Kirsebom et al., Phys. Rev. Lett. 108, 202501 (2012).

[35] C. Giunti, Y. F. Li, C. A. Ternes, and Y. Y. Zhang, Phys. Lett. B 816, 136214 (2021).

[36] A. Serebrov and R. Samoilov, JETP Lett. 112, 199 (2020).

[37] H. Almazán et al., arXiv:2006.13147.

[38] P. Coloma, P. Huber, and T. Schwetz, Eur. Phys. J. C 81, 2 (2021).

[39] M. Tanabashi et al. (Particle Data Group), Phys. Rev. D 98, (030001) (2018); and 2019 update http://pdg.lbl.gov/2019/ reviews/rpp2019-rev-neutrino-mixing.pdf, pp. 42-47.

[40] S. Haystotz et al. arXiv:2003.02289.

[41] M. Tanabashi et al. (Particle Data Group), Phys. Rev. D 98, 030001 (2018); http://pdg.lbl.gov/2019/reviews/rpp2019rev-neutrinos-in-cosmology.pdf.

[42] D. S. Gorbunov, Phys.-Usp. 57, 5 (2014).

[43] M. Aker et al. (KATRIN Collaboration), Phys. Rev. Lett. 123, 221802 (2019).

[44] Y. Kermaïdic (GERDA Collaboration), The XXIX International Conference on Neutrino Physics and Astrophysics Neutrino-2020, https://indico.fnal.gov/event/ 43209/contributions/187846/attachments/129106/159515/ 20200701_Nu2020_Ge76_YoannKermaidic.pdf.

[45] R. Samoilov (Neutrino-4 collaboration), LXX International conference "NUCLEUS-2020. Nuclear physics and elementary particle physics, Nuclear physics technologies https://indico.cern.ch/event/839985/contributions/4060591/ attachments/2124912/3577374/Samoilov_neutrino-4_nucleus .pdf. 